\title{
Chemotherapy alone versus chemotherapy plus radiotherapy for early stage Hodgkin lymphoma (Review)
}

Herbst C, Rehan FA, Skoetz N, Bohlius J, Brillant C, Schulz H, Monsef I, Specht L, Engert A

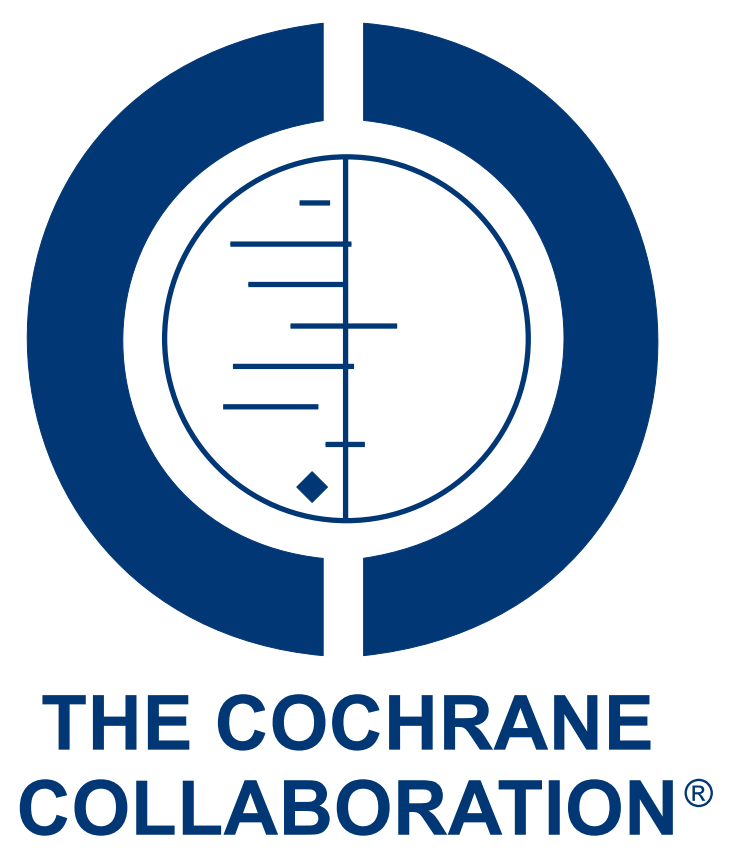

This is a reprint of a Cochrane review, prepared and maintained by The Cochrane Collaboration and published in The Cochrane Library 2011, Issue 3

http://www.thecochranelibrary.com

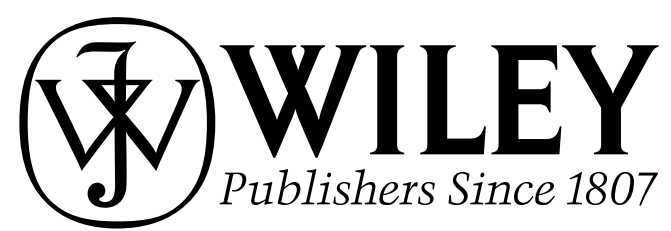

Chemotherapy alone versus chemotherapy plus radiotherapy for early stage Hodgkin lymphoma (Review)

Copyright $\odot$ 20II The Cochrane Collaboration. Published by John Wiley \& Sons, Ltd. 
TABLE OF CONTENTS

HEADER . . . . . . . . . . . . . . . . . . . . . . . . . . . . . . . . . . . . . . . 1

ABSTRACT .. . . . . . . . . . . . . . . . . . . . . . . . . . . . . . . . . . . . . . . . . . . . . . . . .

PLAIN LANGUAGE SUMMARY . . . . . . . . . . . . . . . . . . . . . . . . . . . . . . . . . . . . $\quad 2$

SUMMARY OF FINDINGS FOR THE MAIN COMPARISON . . . . . . . . . . . . . . . . . . . . . . . 2

BACKGROUND . . . . . . . . . . . . . . . . . . . . . . . . . . . . . . . . . . . . 6

OBJECTIVES . . . . . . . . . . . . . . . . . . . . . . . . . . . . . . . . . . . . . . . . . 7

METHODS . . . . . . . . . . . . . . . . . . . . . . . . . . . . . . . . . . . . . . 47

RESULTS . . . . . . . . . . . . . . . . . . . . . . . . . . . . . . . . . . . . . . . 9

Figure 1. . . . . . . . . . . . . . . . . . . . . . . . . . . . . . . . . . . . . . 10

Figure $2 . \quad$. . . . . . . . . . . . . . . . . . . . . . . . . . . . . . . . . . . . . 15

Figure 3. . . . . . . . . . . . . . . . . . . . . . . . . . . . . . . . . . . . . . 17

Figure $4 . \quad$. . . . . . . . . . . . . . . . . . . . . . . . . . . . . . . . . . . . . 17

Figure 5. . . . . . . . . . . . . . . . . . . . . . . . . . . . . . . . . . . . . . 18

Figure 6. . . . . . . . . . . . . . . . . . . . . . . . . . . . . . . . . . . . . . 18

Figure $7 . \quad$. . . . . . . . . . . . . . . . . . . . . . . . . . . . . . . . . . . . . 19

Figure 8. . . . . . . . . . . . . . . . . . . . . . . . . . . . . . . . . . . . . . 19

Figure 9. . . . . . . . . . . . . . . . . . . . . . . . . . . . . . . . . . . . . . 20

Figure 10. . . . . . . . . . . . . . . . . . . . . . . . . . . . . . . . . . . . . . 20

Figure 11. . . . . . . . . . . . . . . . . . . . . . . . . . . . . . . . . . . . . . 21

Figure 12. . . . . . . . . . . . . . . . . . . . . . . . . . . . . . . . . . . . . . 22

Figure 13. . . . . . . . . . . . . . . . . . . . . . . . . . . . . . . . . . . . . . 22

Figure 14. . . . . . . . . . . . . . . . . . . . . . . . . . . . . . . . 24

Figure 15. . . . . . . . . . . . . . . . . . . . . . . . . . . . . . . . . . . . . . 25

Figure 16. . . . . . . . . . . . . . . . . . . . . . . . . . . . . . . . . . . . . . . . . . . 26

Figure 17. . . . . . . . . . . . . . . . . . . . . . . . . . . . . . . . . . . . . . 26

Figure 18. . . . . . . . . . . . . . . . . . . . . . . . . . . . . . . . . . . . . . $\quad 27$

Figure 19. . . . . . . . . . . . . . . . . . . . . . . . . . . . . . . . . . . . . . 27

Figure 20.

Figure $21 . \quad$. . . . . . . . . . . . . . . . . . . . . . . . . . . . . . . . . . . . . 28

Figure 22. . . . . . . . . . . . . . . . . . . . . . . . . . . . . . . . . . . . . . 29

Figure 23. . . . . . . . . . . . . . . . . . . . . . . . . . . . . . . . . . . . . . 29

Figure $24 . \quad$. . . . . . . . . . . . . . . . . . . . . . . . . . . . . . . . . . . . . . . . . . . . .

Figure 25. . . . . . . . . . . . . . . . . . . . . . . . . . . . . . . . . . . . . . 31

Figure 26. . . . . . . . . . . . . . . . . . . . . . . . . . . . . . . . . . . . . . 31

Figure 27. . . . . . . . . . . . . . . . . . . . . . . . . . . . . . . . . . . . . . 32

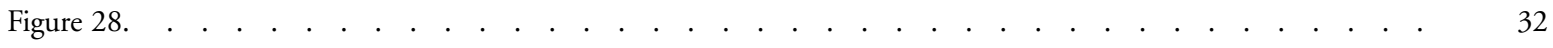

Figure 29. . . . . . . . . . . . . . . . . . . . . . . . . . . . . . . . . . . . . . 33

DISCUSSION . . . . . . . . . . . . . . . . . . . . . . . . . . . . . . . . . . . . .

AUTHORS' CONCLUSIONS . . . . . . . . . . . . . . . . . . . . . . . . . . . . . . . . . . . . 38

ACKNOWLEDGEMENTS . . . . . . . . . . . . . . . . . . . . . . . . . . . . . . . . . . . . . . . . . . . . . 38

REFERENCES . . . . . . . . . . . . . . . . . . . . . . . . . . . . . . . . . . . . . 38

CHARACTERISTICS OF STUDIES . . . . . . . . . . . . . . . . . . . . . . . . . . . . . . . . . . . . . . . . 42

DATA AND ANALYSES . . . . . . . . . . . . . . . . . . . . . . . . . . . . . . . . . . . . . . . . . . . . . $\quad 58$

Analysis 1.1. Comparison 1 Overall Survival, Outcome 1 All trials. . . . . . . . . . . . . . . . . . . . . . 60

Analysis 1.2. Comparison 1 Overall Survival, Outcome 2 Excluding the trial with highest weight (Aviles). . . . . 61

Analysis 1.3. Comparison 1 Overall Survival, Outcome 3 Proportion of patients early favourable. . . . . . . . . 62

Analysis 1.4. Comparison 1 Overall Survival, Outcome 4 Bulky vs non-bulky. . . . . . . . . . . . . . . . . . . 63

Analysis 1.5. Comparison 1 Overall Survival, Outcome 5 Timing of radiotherapy. . . . . . . . . . . . . . . . . . 64

Analysis 1.6. Comparison 1 Overall Survival, Outcome 6 Type of radiotherapy. . . . . . . . . . . . . . . . . . . . 65

Analysis 1.7. Comparison 1 Overall Survival, Outcome 7 Type of chemotherapy. . . . . . . . . . . . . . . . . . 66

Analysis 1.8. Comparison 1 Overall Survival, Outcome 8 Length of follow-up. $\quad$. . . . . . . . . . . . . . . . . . . $\quad$. 67

Chemotherapy alone versus chemotherapy plus radiotherapy for early stage Hodgkin lymphoma (Review)

Copyright $(201$ I The Cochrane Collaboration. Published by John Wiley \& Sons, Ltd. 
Analysis 1.9. Comparison 1 Overall Survival, Outcome 94 year survival in the CT group. . . . . . . . . . . . $\quad . \quad 68$

Analysis 1.10. Comparison 1 Overall Survival, Outcome 10 Allocation concealment. . . . . . . . . . . . . . . 69

Analysis 1.11. Comparison 1 Overall Survival, Outcome 11 ITT-analysis. . . . . . . . . . . . . . . . . . 70

Analysis 2.1. Comparison 2 Progression-Free Survival, Outcome 1 All trials. . . . . . . . . . . . . . . . . . 71

Analysis 2.2. Comparison 2 Progression-Free Survival, Outcome 2 Type of chemotherapy. . . . . . . . . . . . . . 71

Analysis 2.3. Comparison 2 Progression-Free Survival, Outcome 3 Proportion of patients early favourable. . . . . $\quad 72$

Analysis 2.4. Comparison 2 Progression-Free Survival, Outcome 4 Definition of progression. . . . . . . . . . . $\quad 73$

Analysis 2.5. Comparison 2 Progression-Free Survival, Outcome 5 Timing of radiotherapy. . . . . . . . . . . . . 74

Analysis 2.6. Comparison 2 Progression-Free Survival, Outcome 6 Type of radiotherapy. . . . . . . . . . . . . . . 75

Analysis 2.7. Comparison 2 Progression-Free Survival, Outcome 7 Length of follow-up. . . . . . . . . . . . . . 76

Analysis 2.8. Comparison 2 Progression-Free Survival, Outcome 8 Allocation concealment. . . . . . . . . . . . . 77

Analysis 2.9. Comparison 2 Progression-Free Survival, Outcome 9 ITT-Analysis. . . . . . . . . . . . . . . . . . 78

Analysis 3.1. Comparison 3 Complete Response Rate, Outcome 1 All trials. . . . . . . . . . . . . . . . . . . 79

Analysis 3.2. Comparison 3 Complete Response Rate, Outcome 2 Type of Chemotherapy. . . . . . . . . . . . . $\quad$. 80

Analysis 3.3. Comparison 3 Complete Response Rate, Outcome 3 ITT-analysis. . . . . . . . . . . . . . . . . . . 81

Analysis 3.4. Comparison 3 Complete Response Rate, Outcome 4 Number of evaluable patients. . . . . . . . . . . $\quad 82$

Analysis 4.1. Comparison 4 Overall Response Rate, Outcome 1 All Trials. . . . . . . . . . . . . . . . . . . 83

APPENDICES . . . . . . . . . . . . . . . . . . . . . . . . . . . . . . . . . . . . . . 83

WHAT'S NEW . . . . . . . . . . . . . . . . . . . . . . . . . . . . . . . . . . . 92

HISTORY . . . . . . . . . . . . . . . . . . . . . . . . . . . . . . . . . . . . . . . 92

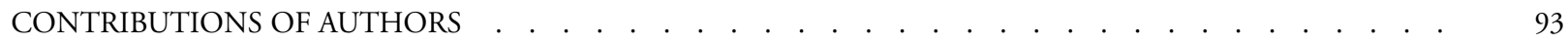

DECLARATIONS OF INTEREST . . . . . . . . . . . . . . . . . . . . . . . . . . . . . . . . . . . . $\quad .93$

SOURCES OF SUPPORT . . . . . . . . . . . . . . . . . . . . . . . . . . . . . . . . . . . . . . . . . . . . 93

DIFFERENCES BETWEEN PROTOCOL AND REVIEW . . . . . . . . . . . . . . . . . . . . . . . . . 93

INDEX TERMS . . . . . . . . . . . . . . . . . . . . . . . . . . . . . . . . . . . . . . . . . . . . 94 


\title{
[Intervention Review]
}

\section{Chemotherapy alone versus chemotherapy plus radiotherapy for early stage Hodgkin lymphoma}

\author{
Christine Herbst ${ }^{2}$, Fareed Ahmed Rehan ${ }^{2}$, Nicole Skoetz ${ }^{1}$, Julia Bohlius ${ }^{3}$, Corinne Brillant ${ }^{1}$, Holger Schulz ${ }^{1}$, Ina Monsef ${ }^{1}$, Lena Specht \\ ${ }^{4}$, Andreas Engert ${ }^{1}$ \\ ${ }^{1}$ Cochrane Haematological Malignancies Group, Department I of Internal Medicine, University Hospital of Cologne, Cologne, \\ Germany. ${ }^{2}$ Cologne, Germany. ${ }^{3}$ Institute of Social and Preventive Medicine, University of Bern, Bern, Switzerland. ${ }^{4}$ Dept. of Oncology, \\ Section 3994, Rigshospitalet, Copenhagen University Hospital, Copenhagen, Denmark
}

Contact address: Andreas Engert, Cochrane Haematological Malignancies Group, Department I of Internal Medicine, University Hospital of Cologne, Kerpener Str. 62, Cologne, 50924, Germany. webmaster@chmg.de. a.engert@uni-koeln.de.

Editorial group: Cochrane Haematological Malignancies Group.

Publication status and date: Edited (no change to conclusions), published in Issue 3, 2011.

Review content assessed as up-to-date: 1 January 2011.

Citation: Herbst C, Rehan FA, Skoetz N, Bohlius J, Brillant C, Schulz H, Monsef I, Specht L, Engert A. Chemotherapy alone versus chemotherapy plus radiotherapy for early stage Hodgkin lymphoma. Cochrane Database of Systematic Reviews 2011, Issue 2. Art. No.: CD007110. DOI: 10.1002/14651858.CD007110.pub2.

Copyright (C) 2011 The Cochrane Collaboration. Published by John Wiley \& Sons, Ltd.

\section{A B S T R A C T}

\section{Background}

Combined modality treatment (CMT) consisting of chemotherapy followed by localised radiotherapy is standard treatment for patients with early stage Hodgkin lymphoma (HL). However, due to long term adverse effects such as secondary malignancies, the role of radiotherapy has been questioned recently and some clinical study groups advocate chemotherapy only for this indication.

\section{Objectives}

We performed a systematic review with meta-analysis of randomised controlled trials (RCTs) comparing chemotherapy alone with CMT in patients with early stage Hodgkin lymphoma with respect to response rate, progression-free survival (alternatively tumour control) and overall survival (OS).

\section{Search strategy}

We searched MEDLINE, EMBASE and CENTRAL as well as conference proceedings from January 1980 to November 2010 for randomised controlled trials comparing chemotherapy alone to the same chemotherapy regimen plus radiotherapy.

\section{Selection criteria}

Randomised controlled trials comparing chemotherapy alone with CMT in patients with early stage HL. Trials in which the chemotherapy differed between treatment arms were excluded. Trials with more than $20 \%$ of patients in advanced stage were also excluded.

\section{Data collection and analysis}

Effect measures used were hazard ratios (HR) for tumour control and OS as well as relative risks for response rates. Two review authors independently extracted data and assessed quality of trials. We contacted study authors to obtain missing information. Since none of the trials reported progression-free survival according to our definitions, all similar outcomes were evaluated as tumour control. 


\section{Main results}

Five RCTs involving 1245 patients were included. The HR was 0.41 ( $95 \%$ confidence interval (CI) 0.25 to 0.66 ) for tumour control and 0.40 (95\% CI 0.27 to 0.61 ) for OS for patients receiving CMT compared to chemotherapy alone. Complete response rates were similar between treatment groups. In sensitivity analyses another six trials were included that did not fulfil the inclusion criteria of our protocol but were considered relevant to the topic. These trials underlined the results of the main analysis.

\section{Authors' conclusions}

Adding radiotherapy to chemotherapy improves tumour control and overall survival in patients with early stage Hodgkin lymphoma.

\section{PLAIN LANGUAGE SUMMARY}

\section{Treatment of early stage Hodgkin lymphoma}

Hodgkin lymphoma is a malignancy of the lymphatic system, first described by Thomas Hodgkin. It can occur in children and adults, but it is more common in the third decade of life. It is one of the most curable forms of cancer. Clinically speaking, there are four stages of Hodgkin lymphoma. Generally, stages I and II are considered as early stage Hodgkin lymphoma and stages III and IV as advanced stage Hodgkin lymphoma. Using risk factors such as presence or absence of bulky disease, age, erythrocyte sedimentation rate and presence or absence of B symptoms, such as night sweats or fever, early stage Hodgkin lymphoma is further classified into early favourable and early unfavourable stages. Treatment options for Hodgkin lymphoma are chemotherapy, radiotherapy or chemotherapy plus radiotherapy. Nowadays chemotherapy plus radiotherapy to involved areas is considered as standard treatment for patients with early stage Hodgkin lymphoma. Radiotherapy has comparatively more treatment related late side effects than chemotherapy, including second malignancies. Perhaps, patients with early stage Hodgkin lymphoma can benefit more by avoiding radiotherapy and can be treated with chemotherapy alone as effectively as with same chemotherapy plus radiotherapy. With this assumption we assess the role of radiotherapy in the treatment of patients with early stage Hodgkin lymphoma. This systematic review compares chance of dying (overall survival) and chance of tumour control in patients with early stage Hodgkin lymphoma after receiving chemotherapy alone or chemotherapy plus radiotherapy. This review includes 1245 patients from five trials in the main analyses. The result of this review is that the addition of radiotherapy to six cycles of chemotherapy is a better treatment option than six cycles of same chemotherapy alone in patients with early stage Hodgkin lymphoma. In terms of five-year tumour control, approximately 5 patients would be needed to treat with chemotherapy plus radiotherapy to prevent one additional relapse or progression in five years. For survival, 11 to 55 patients (depending on the risk of death) require treatment with additional radiotherapy to prevent one death in five years. Therefore chemotherapy plus radiotherapy (combined modality treatment) is superior to the identical chemotherapy alone in patients with early stage Hodgkin lymphoma. 


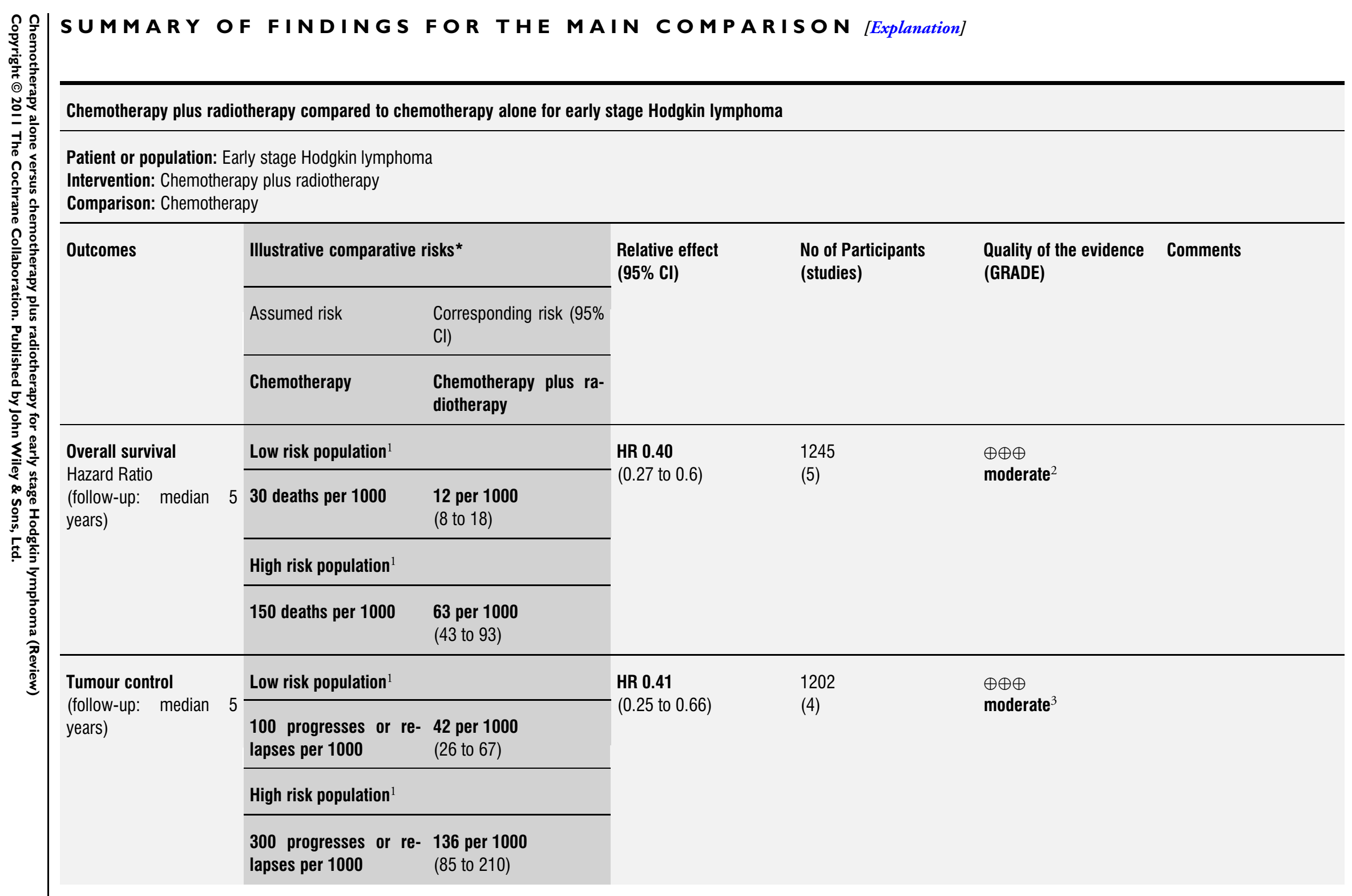




\begin{tabular}{|c|c|c|c|c|c|c|}
\hline \multirow{4}{*}{$\begin{array}{l}\text { Complete } \\
\text { Rate }\end{array}$} & \multicolumn{2}{|l|}{ Low risk population ${ }^{4}$} & \multirow{4}{*}{$\begin{array}{l}\text { RR } 1.07 \\
(0.98 \text { to } 1.17)\end{array}$} & \multirow{4}{*}{$\begin{array}{l}653 \\
(4)\end{array}$} & \multirow{4}{*}{$\begin{array}{l}\oplus \oplus \oplus \\
\text { moderate }^{5}\end{array}$} & \\
\hline & $\begin{array}{l}960 \text { complete responses } \\
\text { per } 1000\end{array}$ & $\begin{array}{l}\mathbf{1 0 2 7} \text { per } \mathbf{1 0 0 0} \\
\text { (941 to } 1123)\end{array}$ & & & & \\
\hline & \multicolumn{2}{|l|}{ High risk population ${ }^{4}$} & & & & \\
\hline & $\begin{array}{l}650 \text { complete responses } \\
\text { per } 1000\end{array}$ & $\begin{array}{l}\mathbf{6 9 6} \text { per } \mathbf{1 0 0 0} \\
\text { (637 to } 760)\end{array}$ & & & & \\
\hline $\begin{array}{l}\text { Long term secondary } \\
\text { malignancies }\end{array}$ & See comment & See comment & Not estimable & - & See comment & $\begin{array}{l}\text { As most of the trials had a } \\
\text { median observation time } \\
\text { of less than } 10 \text { years, long } \\
\text { term information on sec- } \\
\text { ondary malignancies can- } \\
\text { not be expected. }{ }^{6}\end{array}$ \\
\hline Acute adverse effects & See comment & See comment & Not estimable & - & See comment & $\begin{array}{l}\text { Acute adverse effects } \\
\text { were reported differently } \\
\text { in the studies and are sim- } \\
\text { ilar in both groups. }\end{array}$ \\
\hline Long term toxicities & See comment & See comment & Not estimable & - & See comment & $\begin{array}{l}\text { Long term toxicities other } \\
\text { than secondary malig- } \\
\text { nancies are not reported. } \\
8\end{array}$ \\
\hline
\end{tabular}

*The basis for the assumed risk (e.g. the median control group risk across studies) is provided in footnotes. The corresponding risk (and its $95 \%$ confidence interval) is based on the assumed risk in the comparison group and the relative effect of the intervention (and its $95 \% \mathrm{Cl}$ ).

Cl: Confidence interval; RR: Risk ratio; HR: Hazard ratio

GRADE Working Group grades of evidence

High quality: Further research is very unlikely to change our confidence in the estimate of effect.

Moderate quality: Further research is likely to have an important impact on our confidence in the estimate of effect and may change the estimate.

Low quality: Further research is very likely to have an important impact on our confidence in the estimate of effect and is likely to change the estimate.

Very low quality: We are very uncertain about the estimate. 
${ }^{1}$ The risk for the low risk population (of patients with early stage Hodgkin lymphoma) was taken from the trial with the lowest rates of tumour control at 5 years the EORTC-GELA H9-F trial. The high risk rate is approximately the risk in the GATLA 9-H-77.

2 The supplemental analyses shifted the effect estimate (see results section). For this reason, the outcome overall survival was downgraded to moderate.

${ }^{3}$ The definitions of tumour control varied between trials. In some trials the choice of tumour control outcome to be reported may have been guided by strength of the effect. These heterogeneous definitions also lead to an imprecise estimate of the effect or may be considered a publication bias. The effect of these heterogeneous definitions is downgraded here. The same cause is not downgraded in another section again.

${ }^{4}$ The complete response rate for the low risk population is the highest CR rate reported in an included trial in this review (MSKCC \#90-44). The rate for the low risk population is the lowest CR rate reported in this review (CALGB 7751). This estimate of high risk may no longer be relevant today, as ABVD produces much higher rates of CR than CVPP:

${ }^{5}$ Note that for trials using only ABVD the RR is 1.02 (0.95 to 1.09).

${ }^{6} \mathrm{~A}$ non-systematic search of cohorts of Hodgkin lymphoma survivors did not reveal any cohorts that presented 20-year secondary malignancy rates for early stage patients treated with chemotherapy alone versus chemotherapy plus radiotherapy. Cohorts of all patients suggest a higher risk of secondary malignancies for patients receiving any type of radiotherapy. The question of secondary malignancies is addressed in another Cochrane review of individual patient data (Franklin 2005). This review reports $4 \%$ versus $5 \%$ cumulative incidence of secondary malignancies at 20 years. This rate is much lower than the rates for patients with advanced stages $(-20 \%)$.

7 Due to diversity in reporting, adverse effects were not meta-analysed. Common diverse effects reported are nausea and vomiting, alopecia, grade III neutropenia and grade I-II thrombopenia. Rare $(<2 \%)$ are bleomycin induced lung disease, infection-related mortality or high grade neurotoxicity.

${ }^{8}$ In cohorts of patients with Hodgkin lymphoma infertility and chronic cardiac disease are relatively common. However information on how frequent these effects are in early stage patients treated with the treatments discussed in this review was not reported. 


\section{B A C K G R O U N D}

\section{Description of the condition}

Hodgkin lymphoma (HL) is one of the most common malignancies in young adults (Swerdlow 2003; Thomas 2002). It is a malignancy of the lymph nodes and lymphatic system with possible involvement of other organs. The disease is rare with an annual incidence of approximately two to three per 100,000 in most countries (DeVita 1997; Diehl 2005; Mauch 1999) and occurs mostly in young people, the incidence being greatest in the third decade of life (Mueller 1999). Factors associated with Hodgkin lymphoma include family history, viral exposures, and immune suppression (Glaser 1996).

Staging of HL is based on the Ann Arbor system (Carbone 1971), with the addition of a definition of bulky disease (largest tumour diameter $>10 \mathrm{~cm}$ ), often referred to as the Cotswold modification (Lister 1989). Information about prognostic factors such as mediastinal mass, other bulky nodal disease, and extent of subdiaphragmatic disease is included in this classification. Generally, $\mathrm{HL}$ is differentiated into early stage HL and advanced stage HL. On the basis of clinical staging and risk factors, patients are usually assigned to early favourable, early unfavourable and advanced stages (Engert 2007; Klimm 2005). However, there are still small differences in the definition of risk factors used and in the classification of certain subgroups of patients among the different study groups in Europe and the USA.

\section{Description of the intervention}

Usually patients with early stage Hodgkin lymphoma receive two to six cycles of ABVD (adriamycin, bleomycin, vinblastine, and dacarbazine) in combination with involved-field radiotherapy (IF-RT) (Diehl 2005; Engert 2007; Meyer 2005; MSKCC trial \#90-44). Depending on the intensity and dose of treatment given, long term complications such as secondary malignancies (Franklin 2005), cardiac disease (Adams 2004) and infertility are common in Hodgkin survivors. For patients with early stage disease, the 20 year cumulative secondary malignancy rate is estimated to be between four percent and 20 percent (Franklin 2005; Ng 2002a). Risk factors for secondary malignancies (and cardiac disease) are the choice and dose of radiotherapy and chemotherapy (Aleman 2003; Bhatia 2003; Dores 2002; Franklin 2005; Green 2000; Ng 2002a; Ng 2002b; Swerdlow 2000; van Leeuwen 2000). Unfortunately, no long-term comparison of combined modality treatment (CMT), consisting of chemotherapy plus radiotherapy, with chemotherapy alone was possible in cohorts of Hodgkin survivors, in part due to the changes in treatment regimens over time $(\mathrm{Ng}$ 2002a). Nonetheless, to avoid additional radiation-induced toxicity, chemotherapy-only treatment for patients with early stage Hodgkin lymphoma has been advocated (Canellos 2005; Meyer 2005a). This notion was supported by two clinical trials comparing combined modality treatment with chemotherapy alone in which no significant survival disadvantage was observed in patients receiving chemotherapy alone (Meyer 2005; MSKCC trial \#90-44). However, one of these trials compared two cycles of chemotherapy plus radiotherapy with four to six cycles of chemotherapy.

\section{How the intervention might work}

Chemotherpay (CT) and radiotherapy (RT) act on differentiating cells, prone to damage, and stop their growth and ultimately damage them, as result tumour mass shrinks. Along with tumour cure normal body cells are also affected after treatment resulting in treatment related side effects.

\section{Biologic basis of chemotherapy}

The most commonly used chemotherapeutic drugs in the treatment of early stage HL are classified as follows.

- Alkylating agents: cyclophospahamide, mechlorethamine, procarbazine, dacarbazine, cisplatine.

- Anti-tumour antibiotics: bleomycine, doxorubicine (adriamycin), epirubicine, etoposide.

- Anti-mitotic agents: vincristine, vinblastine.

- Steroid hormones: prednisone.

Alkylating agents and anti-tumour antibiotics are phase-nonspecific chemotherapeutic drugs, which can injure DNA at any phase of cell cycle but appear to then block in S-phase or G2 at a check point in a cell cycle before cell division (Sausville 2005). Anti-mitotic agents and steroid hormones are phase-specific chemotherapeutic drugs. Anti-mitotic agents act in M-phase and prevent tumour cell division by destroying mitotic spindle and anti-metabolites act in S-phase and prevent replication of tumour cell's DNA, stopping tumour cell proliferation. Steroid hormones act in Mphase by suppressing the mitosis in lymphocytes (Chaber 2006).

\section{Biologic basis of radiotherapy}

In the target tumour tissue (lymph nodes), RT directly damages the DNA of tumour cells and prevents further cell differentiation of tumour cells and shrinks the tumour mass. Indirectly, it generates free radicals from cell water that are capable of damaging DNA, cell membranes, proteins and organelles. Although radiation can interfere with many cellular processes, many experts feel that cell must undergo a double stranded DNA break from radiation in order to be killed (Hahn 2005). RT is effective when it exerts greater cytotoxic effects on tumour cells than on normal tissues and/or the cells of the normal tissues are more capable of repairing the radiation damage than the tumour cells. 


\section{Why it is important to do this review}

In recent years, a concept of minimal curative therapy with greatest efficacy and least toxicity has emerged in the treatment of early stage HL (Connors 2001; Connors 2005). This concept is based on the assumption that avoidance of RT would result in fewer deaths from late-effects and the long-term survival would be at least comparable and possibly better for patients treated with CT alone in early stage HL. To test this assumption, we performed a systematic review with meta-analysis of randomised controlled trials comparing chemotherapy alone with chemotherapy plus radiotherapy in patients with early stage Hodgkin lymphoma with respect to adverse events, response rate, progression-free survival or similar outcomes and overall survival (OS).

\section{O B JE C T IVES}

The objective of the review was to analyse the efficacy of CTalone compared to CMT in patients with early favourable and early unfavourable stage HL (clinical stage (CS) I and CS II), with respect to OS as primary outcome measure and progressionfree survival (PFS) or similar, response rate and adverse events as secondary outcome measures. Outcomes similar to progressionfree survival are called "tumour control" in this review.

\section{METHODS}

\section{Criteria for considering studies for this review}

\section{Types of studies}

We included RCTs comparing CT-alone with combined modality treatment (CMT) consisting of chemotherapy plus radiotherapy in newly diagnosed patients with CS I and CS II HL (early favourable and early unfavourable stages of HL). We excluded RCTs comparing CT-alone with CMT in patients with all stages of HL if more than $20 \%$ of patients had advanced disease. We used the risk factor definitions as described in the individual trials. The terms "early stage" and "limited-stage" were considered equivalent. We excluded quasi randomised trials. Trials including fewer than 10 participants per arm were also excluded according to the protocol. No such trials were found.

\section{Types of participants}

We included both male and female patients of all ages, with newly confirmed diagnosis of early stage HL (CS I and II) without any prior treatment for HL. If there were more than $20 \%$ of the participants with advanced stage, the trial was excluded. Post hoc, we included such trials in sensitivity analyses. If possible we used subgroup data of early stages.

\section{Types of interventions}

We compared CT (single agent or multiple agent, regardless of dose, number of cycles and intervention time) and both CT plus RT (regardless of dose, field used and intervention time) as primary treatment for patients with CS I and CS II HL (early favourable and early unfavourable stages of HL). We excluded trials if the CT regimen was not identical in both study arms. Post hoc, we included such trials in sensitivity analyses of overall survival and tumour control.

\section{Types of outcome measures}

\section{Primary outcomes}

We evaluated overall survival as the primary endpoint. The preferred definition of OS was "time from entry onto the clinical trial until death as a result of any cause" (Cheson 2007).

\section{Secondary outcomes}

Overall response rate (ORR) and complete response (CR) were evaluated as secondary endpoints. The definitions of overall response and complete response were used as given in the publication. If only complete response and partial response were given, the overall response rate was calculated as complete response plus partial response. Further, we also planned to compare progressionfree survival (PFS) between CT-alone and CMT for patients with early stage HL. Because no trials reported PFS according to our definition (time to tumour progression, relapse or death), we accepted other tumour control outcomes and evaluated these. Finally we evaluated all reported adverse events.

\section{Search methods for identification of studies}

We used search methods as suggested in the Cochrane Handbook for Systematic Reviews of Interventions (Higgins 2008) and by the Cochrane Haematological Malignancies Group (CHMG). No language restriction was applied to reduce the language bias, especially English language bias, as studies showing an intervention to be effective are more likely to be published in English (Dickersin 1993; Egger 1997; Juni 2002). We designed a search strategy with the assistance of the Trials Search Co-ordinator of CHMG for health-related bibliographic databases. 


\section{Electronic searches}

We searched Cochrane Central Register of Controlled Trials (CENTRAL) (see Appendix 1) and MEDLINE (see Appendix 2) for the years January 1977 until November 2010. We searched EMBASE (see Appendix 3) for the years January 1977 until February 2009.

\section{Searching other resources}

We hand-searched the proceedings of the American Society of Hematology for the years 1977 to 2009, except for the year 1979. We searched abstracts of the American Society of Clinical Oncology (ASCO) from 1977 to 2010, except for the year 1990. Proceedings of the International Symposium on Hodgkin Lymphoma (IHSL) were searched from 2004 on. Additionally, two authors performed a cross reference search (screening) of references in primary studies and review articles and checked databases of ongoing clinical trials.

\section{Data collection and analysis}

Two authors independently extracted data from full text publications or from abstract publication and assessed the quality of trials.

\section{Selection of studies}

Two authors independently screened the titles and abstracts of potentially relevant RCTs comparing CT-alone with CMT (with identical CT regimen in both arms) in newly diagnosed patients with CS I and CS II (favourable and early unfavourable stages of HL) according to predefined criteria. Both authors identified eligible trials using a "study eligibility form" which included the following eligibility criteria:

1. Is the study described as randomised?

2. Did $\geq 80 \%$ of the included participants have early stage HL?

3. Were comparison arms treated with CT-alone in one arm and CMT in other arm or arms?

4. Was the same CT regimen used in the comparison arms?

5. Did the study document OS, tumour control (progressionfree survival or similar) or response rate as outcome measure? Studies that met the above mentioned inclusion criteria from screening titles and abstracts were retrieved as full text publications for detailed evaluation. We referred any disagreement between authors to a third author and a decision was made by consensus.

\section{Data extraction and management}

We obtained the full text versions of the publications and abstracts of selected trials for data extraction.

\section{Assessment of risk of bias in included studies}

Two authors independently assessed the risk of bias (quality) in included trials. Please note that this assessment differed from the one proposed in the protocol in order to comply with the new Cochrane Handbook for Systematic Reviews of Interventions. We assessed the following domains:

1. Was the allocation sequence adequately generated?

2. Was allocation adequately concealed?

3. Was knowledge of allocated intervention adequately prevented during trial from outcome assessors?

4. Were incomplete outcome data adequately addressed?

5. Are reports of the trial free of suggestion of selective outcome reporting?

6. Was the trial apparently free of other problems that could put it at risk of bias (e.g. similarity of patients' characteristics at baseline)

We referred any disagreement between reviewers to a third reviewer and a decision was made by consensus. Trials were not assessed blind, since the review authors knew the study author's name, institution, source of publication and results of publication.

\section{Measures of treatment effect}

\section{Time-to-event data}

Treatment effect measures of individual trials were estimated as hazard ratios (HRs) for OS and tumour control from survival analysis, using methods described by Parmar 1998 and Tierney 2007. As no hazard ratios were reported, the HRs for OS and tumour control were estimated indirectly, using logrank statistics through reported $\mathrm{P}$ values and number of events in comparison arms. If $\mathrm{P}$ values were not reported, we estimated HRs for OS and tumour control using survival curves data. Finally, log HRs with standard errors (SEs) were calculated and entered in RevMan 5 for analysis.

In sensitivity analyses, we included additional trials. For some of these trials there were insufficient data (Nachman 2002; Picardi 2007) for tumour control for the calculation of HRs. These HR estimates were improper as they were calculated from the RR and without any time-to-event information. The analysis was repeated without the improper estimates.

\section{Dichotomous data}

The treatment effect measures of individual trials for ORR and $\mathrm{CR}$ were calculated as relative risks (RRs).

\section{Dealing with missing data}

Missing outcome data 
Each trial was assessed for missing patients after randomisation due to drop outs, participants lost to follow-up or protocol violations and number of patients not included in the primary (OS) and secondary outcomes (tumour control and response rates) analyses were calculated. Trials excluding more than $10 \%$ of the randomised patients from outcomes analyses were included in the review and a sensitivity analysis was performed to check the robustness of the results.

\section{Missing information}

To obtain missing information, we contacted study authors and checked previous trial reports of the same investigators.

\section{Assessment of heterogeneity}

A P value of the homogeneity test $\left(\mathrm{Chi}^{2}\right.$ test) was used to identify the statistical heterogeneity between trials with the significance level being set at $\mathrm{P}<0.1$. The extent of inconsistency (heterogeneity) across the trials was quantified by performing $\mathrm{I}^{2}$ statistics in the meta-analyses (Higgins 2002; Higgins 2003). A value of $I^{2} \geq$ $50 \%$ was considered as substantial heterogeneity across the trials in the meta-analysis.

\section{Assessment of reporting biases}

We drew a funnel plot to detect the reporting (publication) and related biases in the meta-analyses that contained at least four included trials. Due to the small number of trials, no linear regression test (Egger 1997a) was performed.

\section{Data synthesis}

We pooled estimated HRs for OS of individual trials using the "Generic Inverse Variance method" using a random-effects model. We pooled estimated relative risks (RRs) for complete response and overall response rate using Der Simonian-Laird method. We calculated the number needed to treat to benefit with 95\% confidence intervals for five-year overall survival or five-year tumour control for hazard ratios as described by Altman 1999.

\section{Subgroup analysis and investigation of heterogeneity}

We planned the following subgroup analyses to investigate the potential causes of heterogeneity with different treatment effects in different groups:

1. Proportion of patients with early favourable stage HL versus early unfavourable stage HL.

2. CS I HL versus CS II HL.

3. Bulky versus non-bulky disease e.g. i) with mediastinal mass versus without mediastinal mass ii) with $>3$ involved nodal areas versus $<3$ involved nodal areas.

4. Different age groups e.g. $<18$ years versus 18 to 50 years versus $>50$ years.
5. Male versus female patients.

6. Different sequence of interventions e.g. CT $+\mathrm{RT}$ versus RT + CT versus CT-RT-CT.

7. Different RT treatment regimens e.g. IF-RT versus extended field radiotherapy (EF-RT).

8. Different CT regimens e.g. ABVD versus CVPP (cyclophosphamide, vinblastine, procarbazine, prednisone) versus EBVP (epirubicin, bleomycin, vinblastine, prednisone).

9. Median length of follow-up (six years or less, more than 6 years)

10. 4-year survival in the CT-alone group $(>90 \%, 80 \%$ to $90 \%)$

Of these planed subgroups, numbers 2, 4, and 5 could not be performed due to lack of data. Post hoc, we performed subgroup analyses including trials that did not fulfil the inclusion criteria of the protocol, but were considered to give information relevant to the review (for a description of these trials see the Results section). We assessed subgroup differences using the test for subgroup differences in RevMan5.

\section{Sensitivity analysis}

We performed a sensitivity analysis to assess the robustness of the overall result with respect to quality and trial design. Using sensitivity analysis we explored:

1. Measures of study quality (ITT-analysis, $>10 \%$ of patients not evaluated vs. $\leq 10 \%$ not evaluated, allocation concealment).

2. The influence of a single large study on the overall result.

\section{R E S U L T S}

\section{Description of studies}

See: Characteristics of included studies; Characteristics of excluded studies; Characteristics of ongoing studies.

\section{Results of the search}

Our literature search produced 2800 potentially relevant references related to the treatment of patients with early stage HL. Of these, 2749 were excluded at the initial stage of screening because they did not fulfil our predefined inclusion criteria. The remaining 51 publications were retrieved as full text publications or abstract publications for detailed evaluation. Of these 51 trials, we excluded 41 and finally 5 trials (10 publications) with 1245 patients were formally included in the main analyses of this review. The overall number of trials screened, identified, selected, excluded and included was documented with reasons according to QUOROM flow diagram (Moher 1999) (Figure 1). 
Figure I. QUOROM-DIAGRAM: Note that the reasons for exclusion are hierarchical, i.e. reasons higher in the list were considered before those lower down.

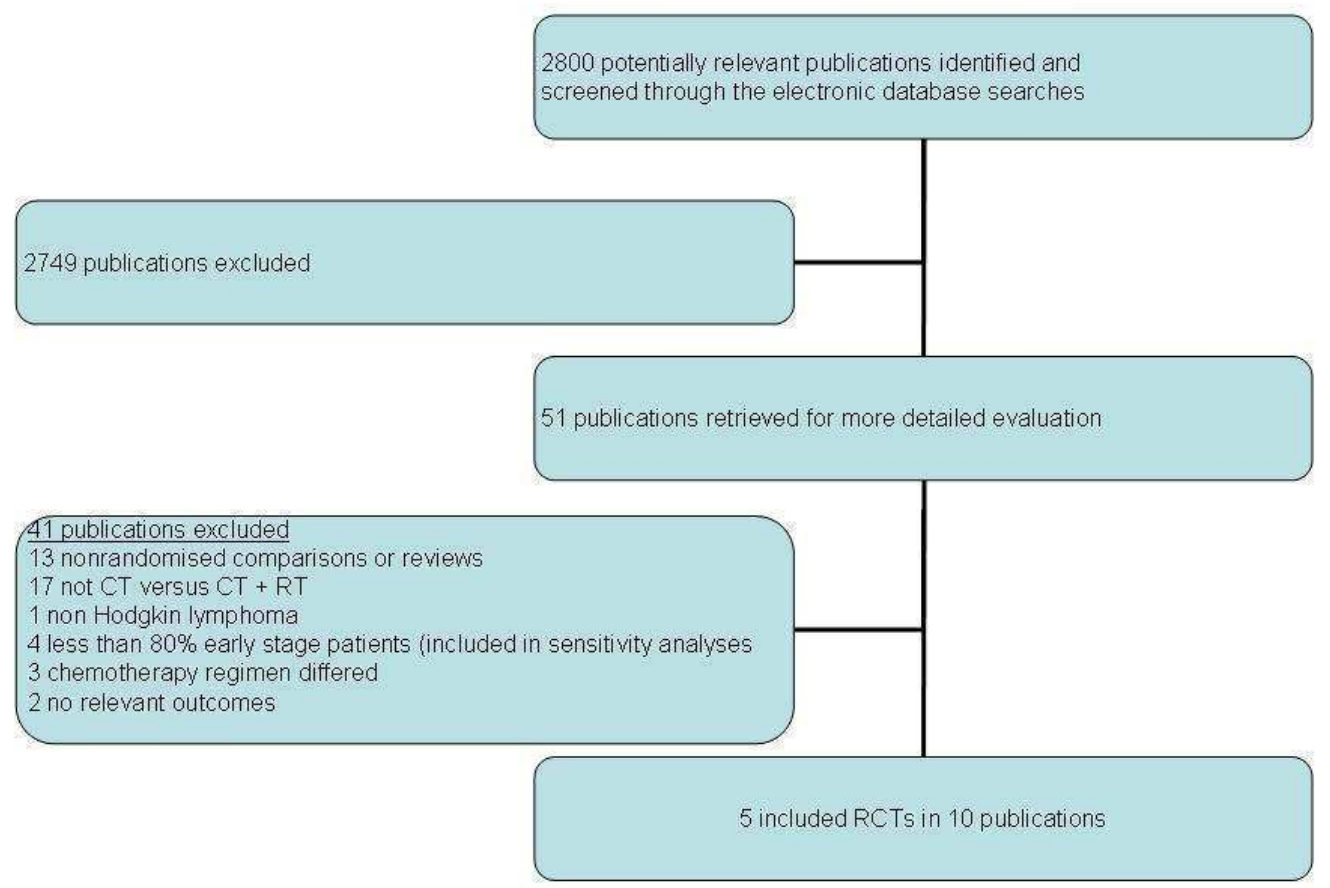

Among the excluded trials (according to our review protocol) six trials were included in sensitivity analyses post hoc, as they yielded relevant information to the underlying clinical question. Five trials included more than $20 \%$ of patients in advanced stages (Kung 2006; Laskar 2004; Nachman 2002; O’Dwyer 1985; Picardi 2007). We identified two trials (Kung 2006; Meyer 2005) in which the patients in the chemotherapy alone group received more cycles of chemotherapy than the patients in the chemotherapy plus radiotherapy group, one of which also included patients of all stages of HL. Characteristics and main results of these trials are described in Table 1.

Table 1. Characteristics of selected excluded trials

\begin{tabular}{|c|c|c|c|c|c|c|c|}
\hline Trial & Patients & $\begin{array}{l}\text { Time of ran- } \\
\text { domisation }\end{array}$ & $\begin{array}{l}\text { Chemother- } \\
\text { apy and radio- } \\
\text { therapy }\end{array}$ & $\begin{array}{l}\text { Subgroup of } \\
\text { early stage pa- } \\
\text { tients? }\end{array}$ & $\begin{array}{l}\text { Length of ob- } \\
\text { servation }\end{array}$ & $\begin{array}{l}\text { Overall } \\
\text { survival }\end{array}$ & $\begin{array}{l}\text { Progres- } \\
\text { sion-free sur- } \\
\text { vival (or event- } \\
\text { free survival) }\end{array}$ \\
\hline
\end{tabular}


Table 1. Characteristics of selected excluded trials (Continued)

\begin{tabular}{|c|c|c|c|c|c|c|c|}
\hline Laskar 2004 & $\begin{array}{l}\text { All stages, age } \\
<70 \text { years }\end{array}$ & in $\mathrm{CR}$ & $\begin{array}{l}6 \text { X ABVD } \\
+ \text { (mainly) IF- } \\
\text { RT vs. } 6 \mathrm{X} \\
\text { ABVD }\end{array}$ & $\begin{array}{l}\text { yes; stage I-II: } \\
\text { CMT: } 55 \\
\text { CT: } 44\end{array}$ & 8 years & $\begin{array}{l}\text { CMT: } 100 \% \\
\text { CT: } 98 \%\end{array}$ & $\begin{array}{l}\text { CMT: } 97 \% \\
\text { CT: } 94 \%\end{array}$ \\
\hline $\begin{array}{l}\text { Nachman } \\
2002\end{array}$ & $\begin{array}{l}\text { Children with } \\
\text { any stage of } \\
\text { HL }\end{array}$ & in $\mathrm{CR}$ & $\begin{array}{l}\text { Early stage: } 4 \mathrm{X} \\
\text { COPP/ABV } \\
+ \\
\text { low dose IF- } \\
\text { RT } \\
\text { vs. } 4 \mathrm{X} \text { COPP/ } \\
\text { ABV }\end{array}$ & $\begin{array}{l}\text { yes; early stage } \\
\text { (I-II) patients: } \\
\text { CMT: } 189 \\
\text { CT: } 173\end{array}$ & 3 years & $\begin{array}{l}100 \% \text { in both } \\
\text { groups }\end{array}$ & $\begin{array}{l}\text { CMT: } 97 \%+/ \\
-1.7(\mathrm{SE}) \\
\text { CT: } 91 \%+/- \\
2.8(\mathrm{SE})\end{array}$ \\
\hline $\begin{array}{l}\text { O’Dwyer } \\
1985 ;\end{array}$ & $\begin{array}{l}\text { stages IB to } \\
\text { IIIA }\end{array}$ & Before CT & $\begin{array}{l}\text { MOPP +RT } \\
\text { vs. MOPP }\end{array}$ & $\begin{array}{l}\text { no; } \\
\text { CMT : } 16 \\
\text { CT: } 17\end{array}$ & 6 years & $\begin{array}{l}\text { CMT: } \\
\text { deaths } \\
\text { CT: } 2 \text { deaths }\end{array}$ & $\begin{array}{l}\text { CMT: } 3 \text { pro- } \\
\text { gression or re- } \\
\text { lapse } \\
\text { CT: } 4 \text { progres- } \\
\text { sion or relapse }\end{array}$ \\
\hline Picardi 2007 & $\begin{array}{l}\text { bulky } \\
\text { HL with resid- } \\
\text { ual masses in } \\
\text { CT that were } \\
\text { PET negative } \\
\text { at restaging af- } \\
\text { ter CT }\end{array}$ & In CR & $\begin{array}{l}6 \text { X VEBEP + } \\
\text { RT vs. } \\
6 \text { X VEBEP }\end{array}$ & $\begin{array}{l}\text { yes; } \\
\text { CMT: } 53 \\
\text { CT: } 52\end{array}$ & 5 years & $\begin{array}{l}100 \% \text { in both } \\
\text { groups }\end{array}$ & $\begin{array}{l}\text { \# of patients } \\
\text { who relapsed: } \\
\text { CMT: } 0 / 53 \\
\text { CT: } 6 / 52\end{array}$ \\
\hline \multicolumn{8}{|c|}{ Trials in which the number of cycles varied between the chemotherapy alone and the chemotherapy plus radiotherapy group } \\
\hline Kung 2006 & $\begin{array}{l}\text { Children, PS } \\
\text { I-IIIA }\end{array}$ & In $\mathrm{CR}$ or $\mathrm{PR}$ & $\begin{array}{l}4 \mathrm{X} \mathrm{MOPP/} \\
\mathrm{ABVD}+\mathrm{IF}- \\
\mathrm{RT} \text { vs. } \\
6 \mathrm{X} \mathrm{MOPP/} \\
\text { ABVD }\end{array}$ & no (31\% IIIA) & 8 years & $\begin{array}{l}\text { CMT: } 96.8 \% \\
+/-2.7 \% \\
\text { CT: } 93.6 \%+/ \\
-3.9 \% \\
\text { P }=0.79\end{array}$ & $\begin{array}{l}\text { CMT: } 91.1 \% \\
+/-4.5 \% \\
\text { CT: } 82.6 \%+/ \\
-5.9 \% \\
\text { P }=0.15\end{array}$ \\
\hline Meyer 2005 & $\begin{array}{l}\text { Early stage (I- } \\
\text { IIA); absence } \\
\text { of bulky dis- } \\
\text { ease }\end{array}$ & Before trial & $\begin{array}{l}2 \times \mathrm{XBVD}+ \\
\text { subtotal nodal } \\
\text { irradiation vs. } \\
\text { 4-6 X ABVD }\end{array}$ & $\begin{array}{l}\text { only early } \\
\text { stage }\end{array}$ & 5 years & $\begin{array}{l}\mathrm{HR}=1.82 \\
{[0.58 \text { to } 5.68]}\end{array}$ & $\begin{array}{l}\mathrm{HR}=0.33 \\
{[0.14 \text { to } 0.80]}\end{array}$ \\
\hline
\end{tabular}

\section{Included studies}

The characteristics of included studies are also summarized in Table 2 . 
Table 2. Characteristics of included trials

\begin{tabular}{|c|c|c|c|c|c|c|}
\hline Trial & $\begin{array}{l}\text { Inclusion crite- } \\
\text { ria }\end{array}$ & $\begin{array}{l}\text { Number of pa- } \\
\text { tients analysed }\end{array}$ & Treatment & $\begin{array}{l}\text { Median fol- } \\
\text { low-up in years } \\
\text { (range) }\end{array}$ & $I T T$-analysis & $\begin{array}{l}\text { Not evaluated or } \\
\text { lost to follow-up } \\
\text { (\%) }\end{array}$ \\
\hline \multirow[t]{2}{*}{ Mexico B2H031 } & \multirow{2}{*}{$\begin{array}{l}\text { CS I to II Supra- } \\
\text { di- } \\
\text { aphragmatic dis- } \\
\text { ease and Bulky } \\
\text { disease }\end{array}$} & 99 & 6xABVD & \multirow[t]{2}{*}{$\begin{array}{lll}11.4 & (6.3 \quad \text { to } \\
16.5) & & \end{array}$} & \multirow[t]{2}{*}{ No } & \multirow[t]{2}{*}{6} \\
\hline & & 102 & $\begin{array}{l}\text { 6xABVD+ IF- } \\
\text { RT }\end{array}$ & & & \\
\hline \multirow{2}{*}{$\begin{array}{l}\text { CALGB } 7751 \\
\text { Interim results }\end{array}$} & \multirow{2}{*}{$\begin{array}{l}\text { "poor prognosis" } \\
\text { PS I or II }\end{array}$} & 18 & 6xCVPP. & \multirow[t]{2}{*}{2 (0 to ?) } & \multirow[t]{2}{*}{ No } & \multirow[t]{2}{*}{32} \\
\hline & & 19 & $\begin{array}{l}\text { 6xCVPP+ IF- } \\
\text { RT }\end{array}$ & & & \\
\hline \multirow[t]{2}{*}{$\begin{array}{l}\text { EORTC-GELA } \\
\text { H9-F } \\
\text { Interim results }\end{array}$} & \multirow{2}{*}{$\begin{array}{l}\text { CS I - II Supra- } \\
\text { diaphrag- } \\
\text { matic disease All } \\
\text { of the favourable } \\
\text { features (age < } \\
50 \text { years, ESR } \\
<50 \mathrm{~mm} / \mathrm{h} \text { or } \\
\text { B symptoms and } \\
\text { ESR }<30 \mathrm{~mm} / \mathrm{h} \text {, } \\
\text { mediastinal- } \\
\text { thoracic ratio < } \\
0.35) \text { CR after } 6 \\
\text { cycles EBVP }\end{array}$} & 130 & $6 x E B V P$ & \multirow[t]{2}{*}{$\begin{array}{l}4.3 \text { years }(1.2 \text { to } \\
6.8)\end{array}$} & \multirow[t]{2}{*}{ Yes } & \multirow[t]{2}{*}{0} \\
\hline & & 448 & $\begin{array}{l}\text { 6x/EBVP }+36 \\
\text { Gy IF-RT or } 20 \\
\text { Gy IF-RT }\end{array}$ & & & \\
\hline \multirow[t]{2}{*}{ GATLA 9-H-77 } & \multirow{2}{*}{$\begin{array}{l}\text { CS I and II, } 173 \\
\text { patients } \\
\text { with favourable } \\
\text { and } 104 \text { patients } \\
\text { with un- } \\
\text { favourable char- } \\
\text { acteristics (age > } \\
45 \text {, sites > 2, } \\
\text { bulky tumour) }\end{array}$} & 142 & 6xCVPP & \multirow[t]{2}{*}{$\begin{array}{l}4 \text { years (not re- } \\
\text { ported) }\end{array}$} & \multirow[t]{2}{*}{ No } & \multirow[t]{2}{*}{6} \\
\hline & & 135 & $\begin{array}{l}6 \mathrm{xCVPP}+\mathrm{IF}- \\
\mathrm{RT}\end{array}$ & & & \\
\hline $\begin{array}{l}\text { MSKCC trial } \\
\# 90-44\end{array}$ & $\begin{array}{l}\text { CS } \\
\text { I to IIIA with- } \\
\text { out bulky dis- } \\
\text { ease, } 13 \% \\
\text { with CS IIIA - } \\
30 \text { to } 50 \% \text { un- } \\
\text { favourable } \\
\text { disease. }\end{array}$ & 76 & 6xABVD & $5.6(0.1$ to 10.4$)$ & $\begin{array}{l}\text { Yes } \\
(\mathrm{OS})\end{array}$ & $\begin{array}{l}0 \text { (OS) } \\
9 \quad(\mathrm{CR}, \text { tumour } \\
\text { control) }\end{array}$ \\
\hline
\end{tabular}


Table 2. Characteristics of included trials (Continued)

76 6xABVD + IF-

Five trials (CALGB 7751; EORTC-GELA H9-F; GATLA 9-H77; Mexico B2H031; MSKCC trial \#90-44) were included in the review. The earliest trial recruited in the 1970s and the latest in 1998 to 2004. The data were extracted from full text publications for four trials and for one trial (EORTC-GELA H9-F) data were extracted from the abstract.

\section{Design}

Of the five included trials, three were two-armed randomised controlled trials and two were three-armed randomised controlled trials. Mexico B2H031 randomised to radiotherapy alone, CMT or $\mathrm{CT}$-alone. The radiotherapy arm was not included in this systematic review. EORTC-GELA H9-F randomised to chemotherapy alone, CMT with 36 Gy or CMT with $20 \mathrm{~Gy}$. The two radiotherapy dosages were evaluated together in this review. There were three multi-centre trials and for two of them (CALGB 7751; GATLA 9-H-77) it was not clear whether they were single centre or multicentre.

\section{Sample sizes}

The smallest trial included 55 (37 analysed) patients (CALGB 7751) and the largest trial 578 patients (EORTC-GELA H9-F).

\section{Location}

The included trials came from a range of research groups from different countries. The trials were conducted in the following countries: one trial in USA (CALGB 7751); one trial in USA and Canada (MSKCC trial \#90-44), one trial in different institutions of European countries (EORTC-GELA H9-F); one trial in Mexico (Mexico B2H031); one trial in Argentina (GATLA 9-H-77).

\section{Participants}

A total of 1245 male and female patients of all ages, with a newly confirmed diagnosis of clinical stage (CS) I and II or pathologic stage (PS) I and II HL and without previous treatment were included. For most patients, histopathologic diagnosis was made

according to Rye modification of Lukes and Butler classification (Lukes 1966).

\section{Interventions}

Patients from included trials were treated with six cycles of CTalone or six cycles of same CT plus radiotherapy (CMT). For included trials, the following CT regimens were used: adriamycin, bleomycin, vinblastine, and dacarbazine (ABVD) in two trials (Mexico B2H031; MSKCC trial \#90-44); CVPP (cyclophosphamide, vinblastine, procarbazine, prednisone) for two trials (CALGB 7751; GATLA 9-H-77); EBVP (epirubicin, bleomycin, vinblastine, prednisone) for one trial (EORTC-GELA H9-F). The size of radiation fields used for the delivery of radiotherapy were as follows: IF-RT in three trials (CALGB 7751; EORTC-GELA H9-F; GATLA 9-H-77); extended-field radiotherapy in two trials (Mexico B2H031; MSKCC trial \#90-44). Two trials (GATLA 9-H-77; Mexico B2H031) administrated three cycles of CT before and after RT (sandwich technique), in the other trials CT was administered prior to RT.

\section{Outcomes}

\section{Primary outcome measure}

All included trials analysed overall survival. The median observation times for overall survival were as follows: 11 years for one trial (Mexico B2H031); seven years for one trial (GATLA 9-H-77); four to five years for two trials (EORTC-GELA H9-F; MSKCC trial \#90-44); 22 months for one trial (CALGB 7751).

\section{Secondary outcome measures}

None of the included trials reported PFS data according to our definition (time to progression or death of any cause). All trials except CALGB 7751 reported some type of progression outcome (see Table 3). Four trials (CALGB 7751; GATLA 9-H-77; Mexico B2H031; MSKCC trial \#90-44) reported response rate. 
Table 3. Definitions of progression outcomes

\begin{tabular}{ll}
\hline Trial & Definition of progression outcome \\
\hline Mexico B2H031 & $\begin{array}{l}\text { Contradictory definitions. In the methods section: "Disease free survival was calculated for CR patients } \\
\text { from the beginning of treatment until clinically or radiologically and biopsy proven relapse." In the results } \\
\text { section the percent disease free were calculated based on the full population. }\end{array}$ \\
\hline EORTC-GELA H9-F & Definition of disease-free survival not reported. (Note all patients are in CR at the time of randomisation.) \\
\hline GATLA 9-H-77 & $\begin{array}{l}\text { Patients who failed to respond were evaluated as relapsed at first month. Patients in CR were evaluated from } \\
\text { date of CR to date of first relapse or death. }\end{array}$ \\
\hline MSKCC trial \#90-44 & Time from enrolment until any progression of disease. \\
\hline
\end{tabular}

\section{Funding}

Academic funding was provided for three trials (CALGB 7751; GATLA 9-H-77; MSKCC trial \#90-44). Source of funding was not described by the remaining two trials.

\section{Conflict of interest}

No trial reported information with respect to conflict of interest.

\section{Excluded studies}

Among the excluded trials (according to our review protocol) a number of trials were included in sensitivity analyses post hoc, as they yielded relevant information to the underlying clinical question. Three trials (566 patients) included patients of all stages and reported some subgroup information for early stage patients (Laskar 2004; Nachman 2002; Picardi 2007). One of these trials examined patients with bulky disease and residual masses after VEBEP chemotherapy who were PET negative (Picardi 2007). One trial included patients in stages I through IIIa which included very few patients (O’Dwyer 1985). Finally we identified two trials in which the patients in the chemotherapy alone group received more cycles of chemotherapy than the patients in the chemotherapy plus radiotherapy group (Kung 2006; Meyer 2005). The chemotherapies used in these trials were ABVD (Laskar 2004; Meyer 2005), COPP/ABV (Nachman 2002), VEBEP (Picardi 2007), MOPP/ ABVD (Kung 2006) and MOPP (O’Dwyer 1985). Their characteristics and main outcomes are presented in Table 1.
For information on excluded trials see Characteristics of excluded studies, where reasons for the exclusion of important excluded trials are listed. For the publication on bleomycin toxicity, where no information for the relevant comparison was found, basic outcomes are also reported.

A total of 41 articles were excluded after detailed evaluation of full text publications. The main reasons for exclusion were:

- 13 non-randomised comparisons or reviews

- 17 not CT versus CMT

- 1 not Hodgkin lymphoma

- 1 only advanced stages

- $4<80 \%$ early stage patients (included in sensitivity analyses)

- 3 chemotherapy regimen differed

- 2 publications of "one trial", where MSKCC patients were randomised to CT vs CMT or different CT plus differing radiotherapy schemes were followed for pulmonary function for approximately one year. The 45 patients with a relevant comparison to this review are presumably included in the MSKCC trial \#90-44. For more details see Characteristics of excluded studies.

Some of these publications are described under Characteristics of excluded studies.

\section{Risk of bias in included studies}

See "risk of bias tables" of included trials and for an overview of the results please see Figure 2. 
Figure 2. Methodological quality summary: review authors' judgements about each methodological quality item for each included study.

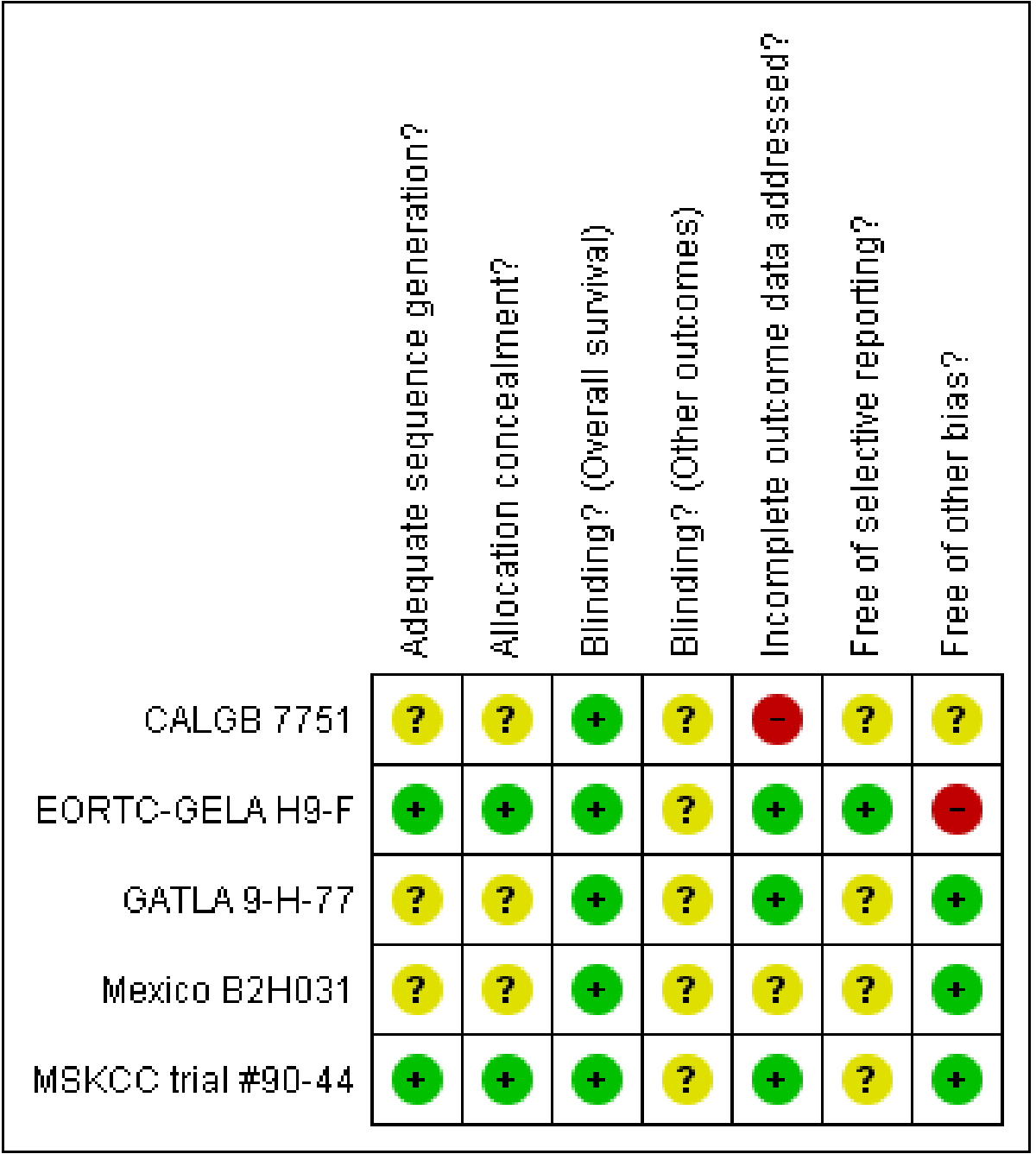

In the EORTC-GELA H9-F trial the chemotherapy alone arm ended early due to stopping rules. Unfortunately it was not possible to receive the data on patients receiving additional radiotherapy only up to the date the chemotherapy alone arm was stopped.

\section{Allocation}

Treatment allocation of patients was performed at a central trials office for one trial (MSKCC trial \#90-44); no information was available for the other trials. No trial reported the sequence generation. Sequence generation and allocation concealment was judged to probably be adequate in large multi-centre trials that have previously adequately randomised and whose allocation was previously adequately concealed (EORTC-GELA H9-F; MSKCC trial \#90-44).

\section{Blinding}

As radiotherapy is difficult to blind, one does not expect the patients to be blinded. However, none of the other trials reported information about blinding of outcome assessors or statisticians. As blinding of the outcome assessors is considered important for this review, all trials were judged "unclear" for the question of blinding.

\section{Incomplete outcome data}

Overall survival 
Most trials described missing outcome data in detail (GATLA 9-H-77) or included all randomised patients in the analysis without reporting any missing data for this outcome (EORTC-GELA H9-F; MSKCC trial \#90-44). In the Mexico B2H031 trial 20/ 327 patients were missing from the analyses without further information (this information was not available only for the two arms included in this review). No trial reported imputation of results for missing data in the context of an intention-to-treat analysis. A high proportion of patients were not analysed in the CALGB 7751 trial but the trial has a low weight in the meta-analysis due to the small number of patients (36 patients evaluated). Due to the small proportion of missing data and the detailed descriptions in most trials, we do not believe that bias was introduced into the meta-analysis by missing data.

For the predefined subgroup analyses we used a strict definition of intention-to-treat analysis (Deeks 2008). Therefore trials that did not include all patients in the analysis and did not describe the method of analysis were not considered to have performed an intention-to-treat analysis (CALGB 7751; GATLA 9-H-77; Mexico B2H031).

\section{Secondary outcomes}

There were more missing data among the secondary outcomes response rate and tumour control.

\section{Selective reporting}

Little information was available about which outcomes were primary outcomes and how these were defined. The choice of progression outcome may be due to selective outcome reporting (see below).

\section{Other potential sources of bias}

None identified.

\section{Effects of interventions}

See: Summary of findings for the main comparison Summary of Findings Table

\section{Primary outcome: Overall survival (OS)}

All five trials of the main analysis with 1245 patients reported OS. The addition of radiotherapy significantly improved OS (HR
$=0.40 ; 95 \%$ CI 0.27 to 0.61 ) with no evidence for heterogeneity between trials $\left(I^{2}=0 \%\right)$; Figure 3 . In three trials the hazard ratios had to be based on the survival curves or reported dates of deaths (CALGB 7751; EORTC-GELA H9-F; Mexico B2H031). In all cases, constant censoring was assumed as described by Tierney 2007 . However, this assumption is problematic for the EORTC-GELA H9-F trial because the no-radiation arm was closed early. Estimating a difference in censoring to account for the premature closure of the chemotherapy alone arm had only a minor effect on the hazard ratio calculated (Table 4 shows HR of Mexico B2H031 and EORTC-GELA H9-F with different censoring assumptions). Other uncertainties in the hazard ratio calculation arose from $\mathrm{P}$ values with only one significant digit. The results of the meta-analysis were dominated by the Mexico B2H031 trial, which had a weight of $50.4 \%$ (Figure 3). When excluding the Mexico B2H031 trial from the meta-analysis in a sensitivity analysis, the summary hazard ratio remained statistically significant favouring CMT $\left(0.57 ; 95 \%\right.$ CI 0.33 to $0.98, \mathrm{I}^{2}$ $=0 \%$ ) (Figure 4). The substantial weight of the Mexico B2H031 trial was due to the higher number of observed deaths occurring with longer follow-up. Here, patients had been followed up for a median of 12 years as compared to two to seven years in the other trials. The four-year survival rate of $83 \%$ in the Mexico B2H031 trial was comparable to those of the other trials ranging from $85 \%$ (MSKCC trial \#90-44) to 87\% (GATLA 9-H-77). No information on the four-year survival was available from CALGB 7751. The EORTC-GELA H9-F trial included early favourable patients in CR only and as a result had a higher five-year survival rate of $97 \%$. None of the subgroup analyses showed statistically significant differences between the subgroups examined (type of chemotherapy $\mathrm{P}=0.14$, early favourable or unfavourable disease $\mathrm{P}=0.31$, bulky or no bulky disease $\mathrm{P}=0.98$, type $(\mathrm{P}=0.20)$ and timing $(P=0.76)$ of radiation therapy. See Figure 5 , Figure 6, Figure 7, Figure 8, Figure 9. Subgroup analyses by age or sex were not possible due to the limited amount of data available. Subgroup differences in performed sensitivity and subgroup analyses were not statistically significant. The $\mathrm{P}$ values for subgroup differences were $\mathrm{P}=0.12$ (length of follow-up Figure 10), $\mathrm{P}=0.82$ (fouryear overall survival in the chemotherapy group Figure 11), $\mathrm{P}=$ 0.56 (allocation concealment Figure 12), P = 0.56 (ITT-analysis Figure 13). Due to the small number of trials included in the metaanalysis true differences between subgroups may be missed in the subgroup and sensitivity analyses. 
Figure 3. Forest plot of comparison: I Overall Survival, outcome: I.I All trials.

\begin{tabular}{|c|c|c|c|c|}
\hline \multirow{2}{*}{$\begin{array}{l}\text { Study or Subgroup } \\
\text { CALGB } 7751\end{array}$} & \multicolumn{2}{|c|}{$\begin{array}{cc}\text { Hazard Ratio } \\
\text { Weight } & \text { N, Random, 95\% } \mathrm{Cl} \\
\end{array}$} & \multicolumn{2}{|c|}{$\begin{array}{c}\text { Hazard Ratio } \\
\text { N, Random, 95\% } \mathrm{Cl}\end{array}$} \\
\hline & $5.4 \%$ & $0.63[0.11,3.65]$ & & \\
\hline EORTC-GELA H9-F & $4.9 \%$ & $0.27[0.04,1.74]$ & & \\
\hline GATLA 9-H-77 & $31.0 \%$ & $0.71[0.35,1.44]$ & & - \\
\hline Mexico B2H031 & $48.9 \%$ & $0.29[0.17,0.51]$ & & \\
\hline MSKCC trial \#90-44 & $9.7 \%$ & $0.31[0.08,1.15]$ & & \\
\hline Total $(95 \% \mathrm{Cl})$ & $100.0 \%$ & $0.40[0.27,0.61]$ & & \\
\hline \multicolumn{3}{|c|}{$\begin{array}{l}\text { Heterogeneity: } \operatorname{Tau}^{2}=0.01 ; \mathrm{Chi}^{2}=4.14, \mathrm{df}=4(\mathrm{P}=0.39) ; \mathrm{I}^{2}=3 \% \\
\text { Test for overall effect: } Z=4.33(\mathrm{P}=0.0001)\end{array}$} & $\begin{array}{lc}1 & 1 \\
0.05 & 0.2 \\
\text { Favours CMT }\end{array}$ & $\begin{array}{cc}\frac{1}{5} & 20 \\
\text { Favours CT-alone }\end{array}$ \\
\hline
\end{tabular}

Figure 4. Forest plot of comparison: I Overall Survival, outcome: I. I I Excluding the trial with highest weight (Mexico B2H03I).

\begin{tabular}{|c|c|c|c|c|c|c|c|c|}
\hline Study or Subgroup & log[Hazard Ratio] & SE & $\begin{array}{l}\text { CMT } \\
\text { Total }\end{array}$ & $\begin{array}{c}\text { CT-alone } \\
\text { Total }\end{array}$ & Weight & $\begin{array}{c}\text { Hazard Ratio } \\
\text { N, Random, 95\% } \mathrm{Cl}\end{array}$ & $\begin{array}{r}\text { Hazard } \\
\text { N, Randon }\end{array}$ & $\begin{array}{l}\text { d Ratio } \\
\mathrm{m}, 95 \% \mathrm{Cl}\end{array}$ \\
\hline CALGB 7751 & -0.31 & 0.68 & 19 & 18 & $16.6 \%$ & $0.73[0.19,2.78]$ & $\longrightarrow$ & \\
\hline EORTC-GELA H9-F & -1.2925 & 0.9426 & 448 & 130 & $8.6 \%$ & $0.27[0.04,1.74]$ & & \\
\hline GATLA $9-\mathrm{H}-77$ & -0.3484 & 0.3651 & 135 & 142 & $57.5 \%$ & $0.71[0.35,1.44]$ & 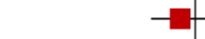 & \\
\hline MSKCC trial \#90-44 & -1.1671 & 0.6667 & 76 & 76 & $17.3 \%$ & $0.31[0.08,1.15]$ & & \\
\hline Total $(95 \% \mathrm{Cl})$ & & & 678 & 366 & $100.0 \%$ & $0.57[0.33,0.98]$ & & \\
\hline \multicolumn{7}{|c|}{$\begin{array}{l}\text { Heterogeneity: } \mathrm{Tau}^{2}=0.00 ; \mathrm{Chi}^{2}=1.90, \mathrm{df}=3(\mathrm{P}=0.59) ; \mathrm{I}^{2}=0 \% \\
\text { Test for overall effect: } Z=2.04(\mathrm{P}=0.04)\end{array}$} & $\begin{array}{l}0.01 \quad 0.1 \\
\text { Favours CMT }\end{array}$ & \begin{tabular}{|c|}
10 \\
Favours CT-alone
\end{tabular} \\
\hline
\end{tabular}


Figure 5. Forest plot of comparison: I Overall Survival, outcome: I.2 Proportion of patients early favourable.

\begin{tabular}{|c|c|c|c|c|c|c|}
\hline Study or Subgroup & log[Hazard Ratio] & SE & Weight & $\begin{array}{l}\text { Hazard Ratio } \\
\text { N, Random, 95\% } \mathrm{Cl}\end{array}$ & $\begin{array}{r}\text { Hazard } \\
\text { N, Randor }\end{array}$ & $\begin{array}{l}\text { d Ratio } \\
\text { om, 95\% } \mathrm{Cl}\end{array}$ \\
\hline \multicolumn{7}{|c|}{ 1.3.1 All patients early favourable } \\
\hline $\begin{array}{l}\text { EORTC-GELA H9-F } \\
\text { Subtotal (95\% Cl) }\end{array}$ & -1.2925 & 0.9426 & $\begin{array}{l}4.9 \% \\
4.9 \%\end{array}$ & $\begin{array}{r}0.27[0.04,1.74] \\
0.27[0.04,1.74]\end{array}$ & & 一 \\
\hline \multicolumn{7}{|c|}{$\begin{array}{l}\text { Heterogeneity: Not applicable } \\
\text { Test for overall effect: } Z=1.37(P=0.17)\end{array}$} \\
\hline \multicolumn{7}{|c|}{$1.3 .2 \sim 1 / 3$ to $1 / 2$ patients early unfawourable } \\
\hline GATLA 9-H-77 & -0.3484 & 0.3651 & $31.0 \%$ & $0.71[0.35,1.44]$ & - & \\
\hline $\begin{array}{l}\text { MSKCC trial \#90-44 } \\
\text { Subtotal (95\% Cl) }\end{array}$ & -1.1671 & 0.6667 & $\begin{array}{r}9.7 \% \\
40.8 \%\end{array}$ & $\begin{array}{r}0.31[0.08,1.15] \\
\mathbf{0 . 5 7}[0.28, \mathbf{1 . 1 5}]\end{array}$ & & \\
\hline \multicolumn{7}{|c|}{$\begin{array}{l}\text { Heterogeneity: } \text { Tau }^{2}=0.05 ; \mathrm{Chi}^{2}=1.16, \mathrm{df}=1(\mathrm{P}=0.28) ; \mathrm{I}^{2}=14 \% \\
\text { Test for overall effect: } Z=1.57(P=0.12)\end{array}$} \\
\hline \multicolumn{7}{|c|}{ 1.3.3 All patients early unfawourable } \\
\hline CALGB 7751 & -0.47 & 0.9 & $5.4 \%$ & $0.63[0.11,3.65]$ & & \\
\hline $\begin{array}{l}\text { Mexico B2 } \mathrm{HO} 031 \\
\text { Subtotal }(95 \% \mathrm{Cl})\end{array}$ & -1.225 & 0.2852 & $\begin{array}{l}48.9 \% \\
54.3 \%\end{array}$ & $\begin{array}{r}0.29[0.17,0.51] \\
\mathbf{0 . 3 1}[\mathbf{0 . 1 8}, \mathbf{0 . 5 4}]\end{array}$ & & \\
\hline \multicolumn{7}{|c|}{$\begin{array}{l}\text { Heterogeneity: } \operatorname{Tau}^{2}=0.00 ; \mathrm{Ch}^{2}=0.64, \mathrm{df}=1(\mathrm{P}=0.42) ; \mathrm{I}^{2}=0 \% \\
\text { Test for overall effect: } Z=4.25(\mathrm{P}<0.0001)\end{array}$} \\
\hline Total $(95 \% \mathrm{Cl})$ & & & $100.0 \%$ & $0.40[0.27,0.61]$ & & \\
\hline \multicolumn{5}{|c|}{$\begin{array}{l}\text { Heterogeneity: } \text { Tau }^{2}=0.01 ; \mathrm{Chi}^{2}=4.14, \mathrm{df}=4(P=0.39) ; I^{2}=3 \% \\
\text { Test for overall effect: } Z=4.33(P<0.0001)\end{array}$} & $\begin{array}{lc} \\
0.01 & 0.1 \\
\text { Favours }\end{array}$ & $\begin{array}{r}10 \\
10 \\
\text { Favours CT-alone }\end{array}$ \\
\hline
\end{tabular}

Figure 6. Forest plot of comparison: I Overall Survival, outcome: I.3 Bulky vs non-bulky.

\begin{tabular}{|c|c|c|c|c|c|c|}
\hline \multirow{2}{*}{$\frac{\text { Stuchy or Subgroup }}{\text { 1.4.1 Bulky disease }}$} & log[Hazard Ratio] & \multirow[t]{2}{*}{ SE } & \multirow[t]{2}{*}{ Weight } & \multirow[t]{2}{*}{$\begin{array}{l}\text { Hazard Ratio } \\
\text { N, Random, 95\% Cl }\end{array}$} & \multicolumn{2}{|c|}{$\begin{array}{l}\text { Hazard Ratio } \\
\text { N, Random, 95\% Cl }\end{array}$} \\
\hline & & & & & & \\
\hline $\begin{array}{l}\text { Mexico B2H031 } \\
\text { Subtotal (95\% Cl) }\end{array}$ & -1.225 & 0.2852 & $\begin{array}{l}78.5 \% \\
78.5 \%\end{array}$ & $\begin{array}{r}0.29[0.17,0.51] \\
\mathbf{0 . 2 9}[0.17,0.51]\end{array}$ & & \\
\hline \multicolumn{7}{|c|}{$\begin{array}{l}\text { Heterogeneity: Not applicable } \\
\text { Test for overall effect: } Z=4.30(P<0.0001)\end{array}$} \\
\hline \multicolumn{7}{|c|}{ 1.4.2 Non-bulky disease } \\
\hline EORTC-GELA H9-F & -1.2925 & 0.9426 & $7.2 \%$ & $0.27[0.04,1.74]$ & & \\
\hline $\begin{array}{l}\text { MSKCC trial \#90-44 } \\
\text { Subtotal (95\% Cl) }\end{array}$ & -1.1671 & 0.6667 & $\begin{array}{l}14.4 \% \\
21.5 \%\end{array}$ & $\begin{array}{r}0.31[0.08,1.15] \\
\mathbf{0 . 3 0}[\mathbf{0 . 1 0 , 0 . 8 7 ]}\end{array}$ & & \\
\hline \multicolumn{7}{|c|}{$\begin{array}{l}\text { Heterogeneity: } \operatorname{Tau}^{2}=0.00 ; \mathrm{Chi}^{2}=0.01, \mathrm{df}=1(P=0.91) ; \mathrm{I}^{2}=0 \% \\
\text { Test for overall effect: } Z=2.22(P=0.03)\end{array}$} \\
\hline Total (95\% Cl) & & & $100.0 \%$ & $0.29[0.18,0.48]$ & & \\
\hline \multicolumn{5}{|c|}{$\begin{array}{l}\text { Heterogeneity: } \text { Tau }^{2}=0.00 ; \mathrm{Chi}^{2}=0.01, \mathrm{df}=2(P=0.99) ;\left.\right|^{2}=0 \% \\
\text { Test for overall effect: } Z=4.84(P=0.00001)\end{array}$} & \begin{tabular}{|cc|c|c|}
0.01 & 0.1 & 1 \\
Favours CMT
\end{tabular} & Favours CT-alone \\
\hline
\end{tabular}


Figure 7. Forest plot of comparison: I Overall Survival, outcome: I.4 Timing of radiotherapy.

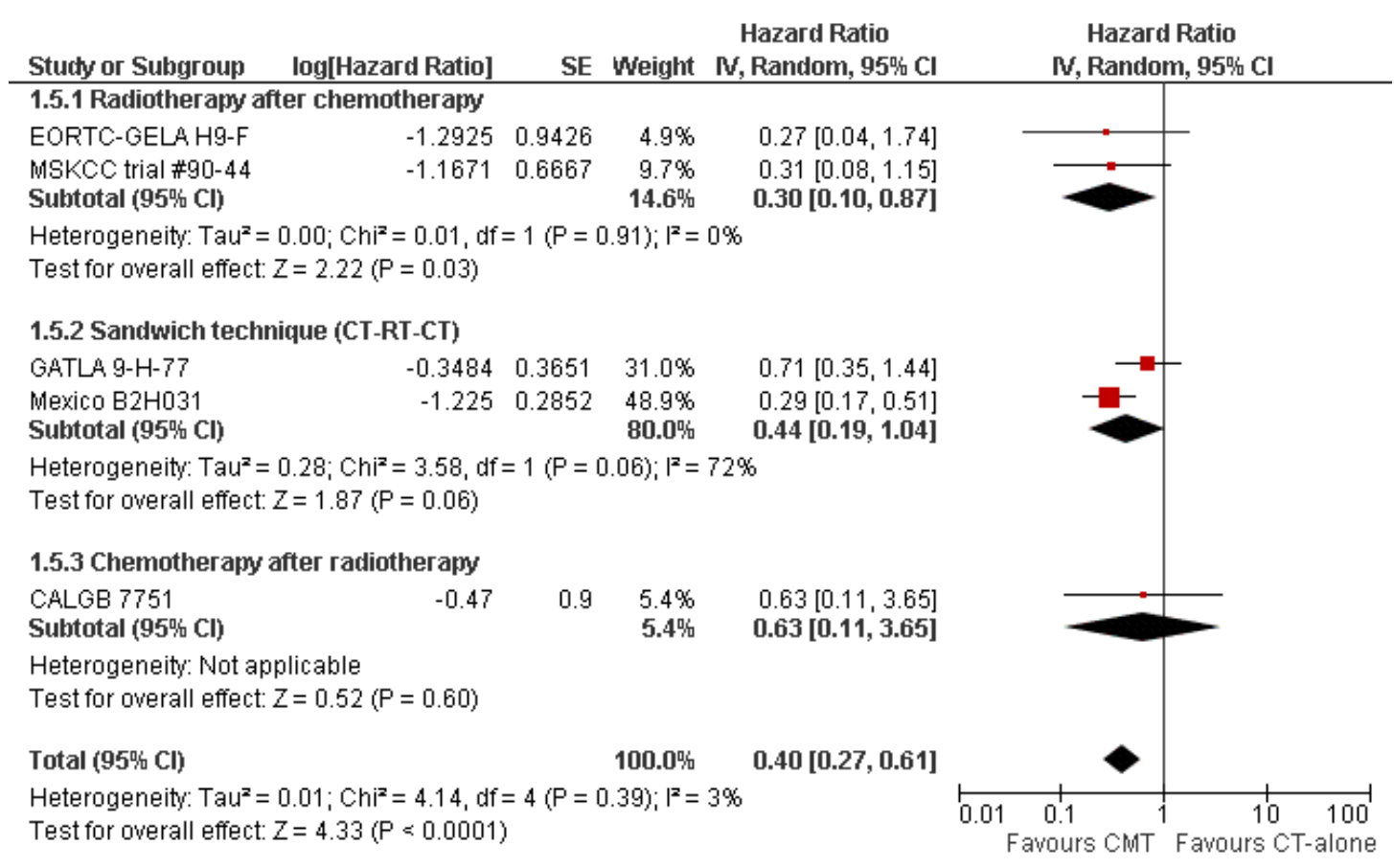

Figure 8. Forest plot of comparison: I Overall Survival, outcome: I.5 Type of radiotherapy.

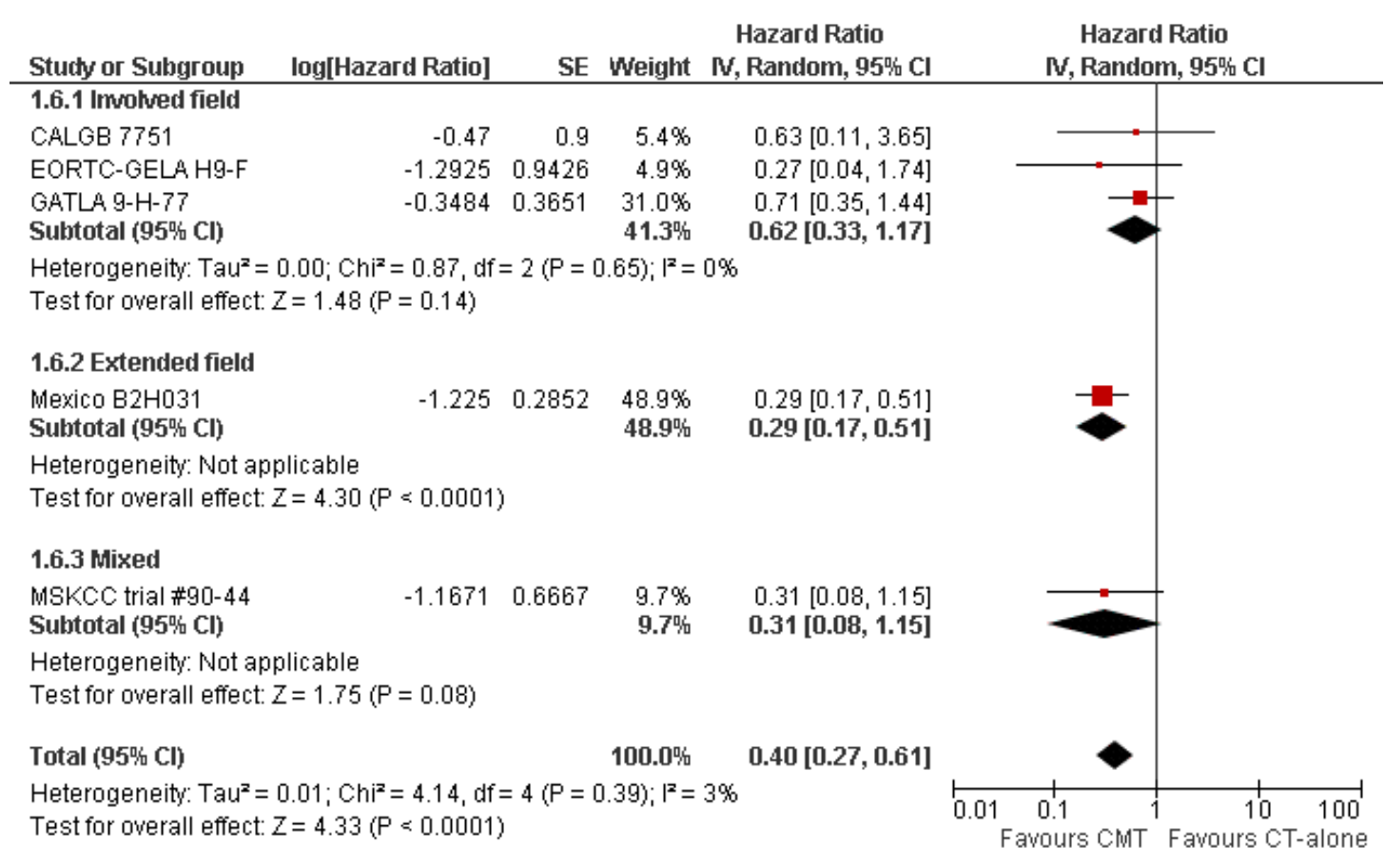


Figure 9. Forest plot of comparison: I Overall Survival, outcome: I.6 Type of chemotherapy.

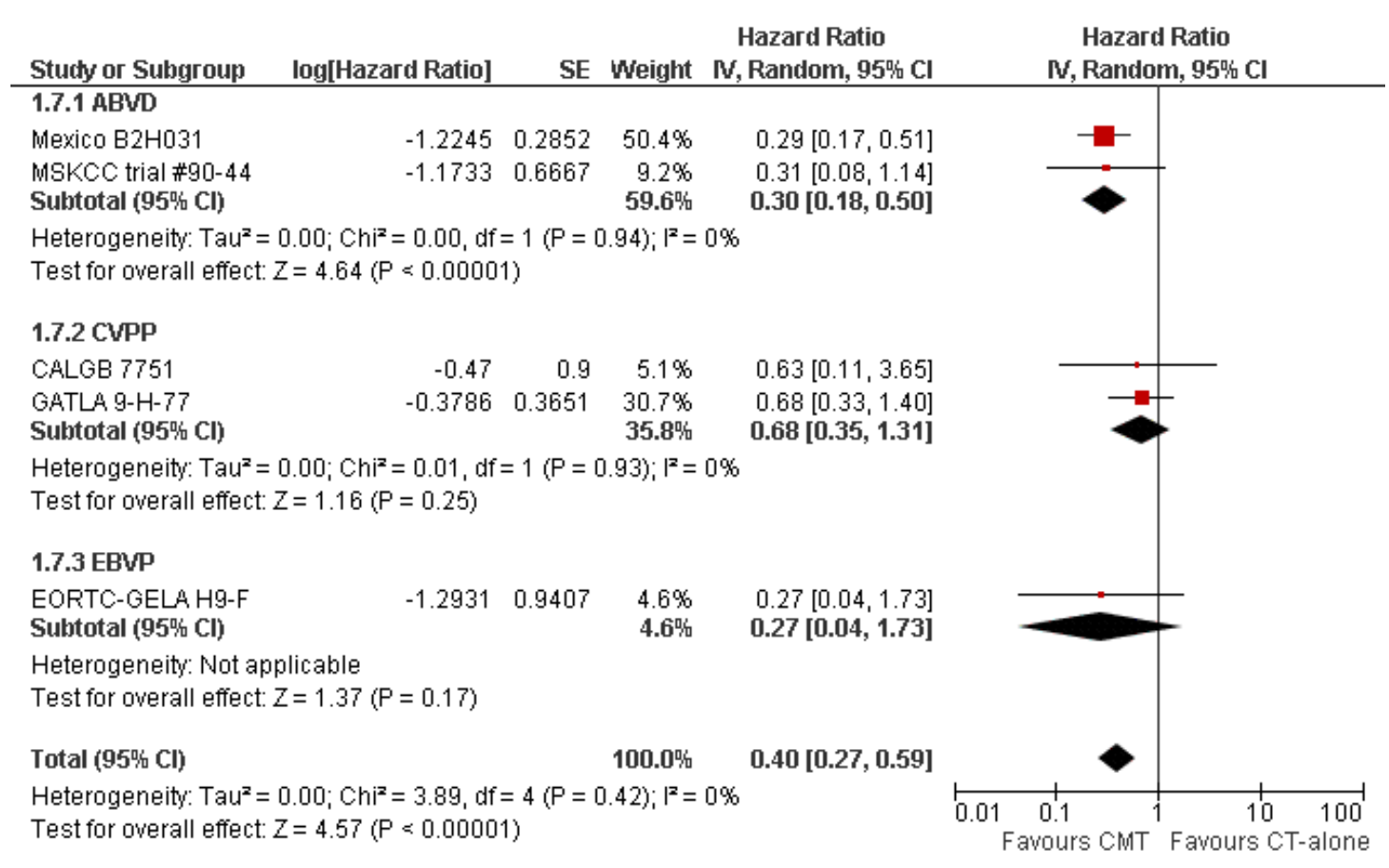

Figure 10. Forest plot of comparison: I Overall Survival, outcome: I.7 Length of follow-up.

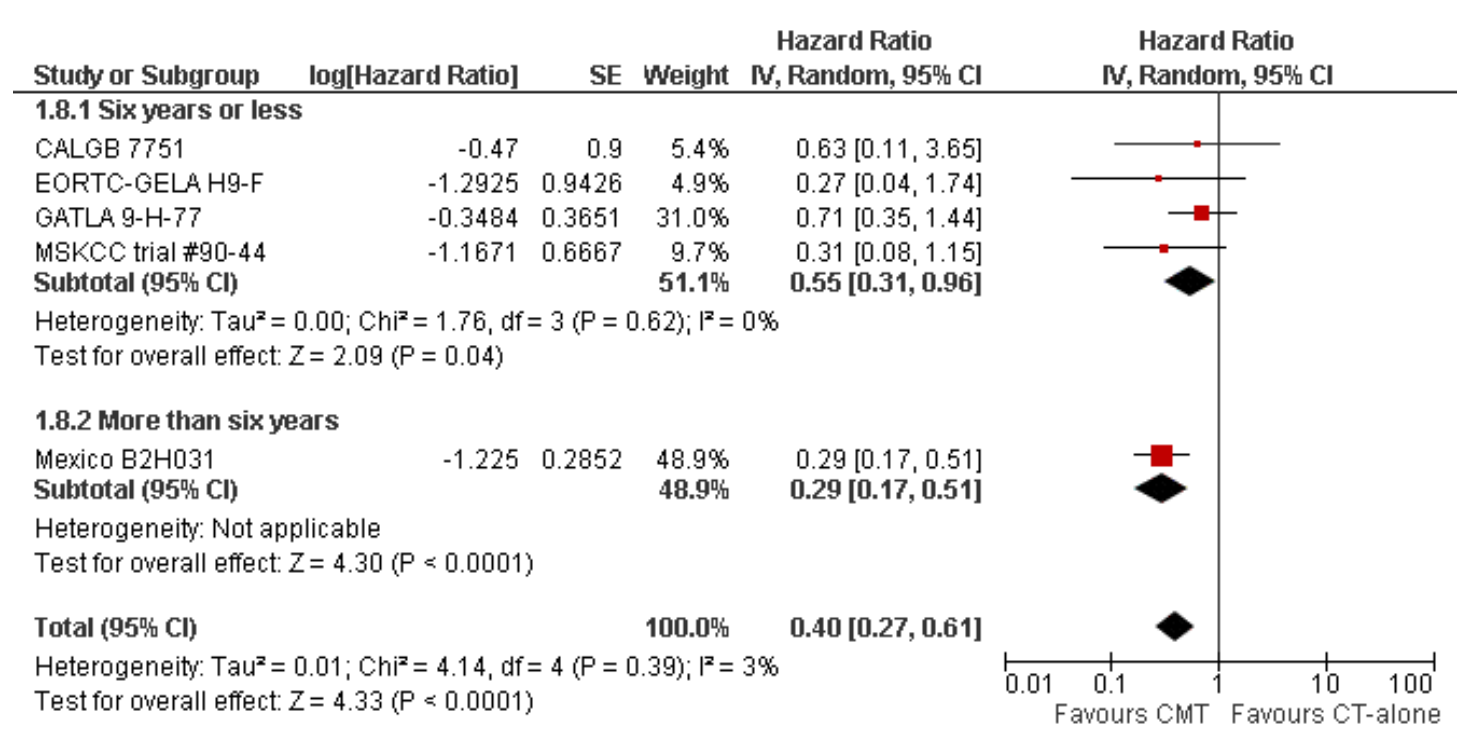


Figure I I. Forest plot of comparison: I Overall Survival, outcome: I.8 4 year survival in the CT group.

\begin{tabular}{|c|c|c|c|c|c|c|}
\hline Study or Subgroup & log[Hazard Ratio] & SE & Weight & $\begin{array}{l}\text { Hazard Ratio } \\
\text { N, Random, 95\% } \mathrm{Cl}\end{array}$ & $\begin{array}{l}\text { Hazard } \\
\text { N, RandoI }\end{array}$ & 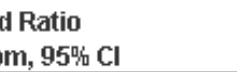 \\
\hline \multicolumn{7}{|l|}{$1.9 .1>90 \%$} \\
\hline $\begin{array}{l}\text { EORTC-GELA H9-F } \\
\text { Subtotal (95\% } \mathrm{Cl})\end{array}$ & -1.2925 & 0.9426 & $\begin{array}{l}4.9 \% \\
4.9 \%\end{array}$ & $\begin{array}{r}0.27[0.04,1.74] \\
\mathbf{0 . 2 7}[0.04,1.74]\end{array}$ & & - \\
\hline \multicolumn{7}{|c|}{$\begin{array}{l}\text { Heterogeneity: Not applicable } \\
\text { Test for overall effect: } Z=1.37(P=0.17)\end{array}$} \\
\hline \multicolumn{7}{|l|}{$1.9 .280 \%-90 \%$} \\
\hline GATLA 9-H-77 & -0.3484 & 0.3651 & $31.0 \%$ & $0.71[0.35,1.44]$ & & \\
\hline Mexico B2H031 & -1.225 & 0.2852 & $48.9 \%$ & $0.29[0.17,0.51]$ & & \\
\hline $\begin{array}{l}\text { MSKCC trial \#90-44 } \\
\text { Subtotal (95\% } \mathrm{Cl})\end{array}$ & -1.1671 & 0.6667 & $\begin{array}{r}9.7 \% \\
89.7 \%\end{array}$ & $\begin{array}{l}0.31[0.08,1.15] \\
\mathbf{0 . 4 1}[0.22,0.77]\end{array}$ & & \\
\hline \multicolumn{7}{|c|}{$\begin{array}{l}\text { Heterogeneity: } \operatorname{Tau}^{2}=0.14 ; \mathrm{Chi}^{2}=3.73, \mathrm{df}=2(\mathrm{P}=0.15) ; \mathrm{I}^{2}=46 \% \\
\text { Test for overall effect: } Z=2.80(\mathrm{P}=0.005)\end{array}$} \\
\hline \multicolumn{7}{|l|}{ 1.9.3 unknown } \\
\hline $\begin{array}{l}\text { CALGB } 7751 \\
\text { Subtotal }(95 \% \text { Cl) }\end{array}$ & -0.47 & 0.9 & $\begin{array}{l}5.4 \% \\
5.4 \%\end{array}$ & $\begin{array}{r}0.63[0.11,3.65] \\
\mathbf{0 . 6 3}[0.11,3.65]\end{array}$ & & \\
\hline \multicolumn{7}{|c|}{$\begin{array}{l}\text { Heterogeneity: Not applicable } \\
\text { Test for overall effect: } Z=0.52(P=0.60)\end{array}$} \\
\hline Total $(95 \% \mathrm{Cl})$ & & & $100.0 \%$ & $0.40[0.27,0.61]$ & & \\
\hline $\begin{array}{l}\text { Heterogeneity: } \operatorname{Tau}^{2}= \\
\text { Test for overall effect: }\end{array}$ & $\begin{array}{l}0.01 ; \mathrm{Chi}^{2}=4.14, \mathrm{df}= \\
Z=4.33(P<0.0001)\end{array}$ & $=4(P=0$ & (1.39); $\left.\right|^{2}=$ & & \begin{tabular}{lc|l}
0.01 & 0.1 & 1 \\
Favours CMT
\end{tabular} & \begin{tabular}{|c|}
10 \\
100 \\
Favours CT-alone
\end{tabular} \\
\hline
\end{tabular}


Figure I2. Forest plot of comparison: I Overall Survival, outcome: I.9 Allocation concealment.

\begin{tabular}{|c|c|c|c|c|c|c|}
\hline Stucty or Subgroup & log[Hazard Ratio] & SE & Weight & $\begin{array}{l}\text { Hazard Ratio } \\
\text { N, Random, 95\% Cl }\end{array}$ & $\begin{array}{r}\text { Hazard } \\
\mathbb{N}, \text { Randor }\end{array}$ & d Ratio \\
\hline \multicolumn{7}{|c|}{ 1.10.1 Allocation concealment unclear } \\
\hline CALGB 7751 & -0.47 & 0.9 & $5.4 \%$ & $0.63[0.11,3.65]$ & & \\
\hline GATLA 9-H-77 & -0.3484 & 0.3651 & $31.0 \%$ & $0.71[0.35,1.44]$ & & \\
\hline $\begin{array}{l}\text { Mexico B2H031 } \\
\text { Subtotal (95\% Cl) }\end{array}$ & -1.225 & 0.2852 & $\begin{array}{l}48.9 \% \\
85.4 \%\end{array}$ & $\begin{array}{r}0.29[0.17,0.51] \\
\mathbf{0 . 4 6}[\mathbf{0 . 2 3}, \mathbf{0 . 8 9}]\end{array}$ & & \\
\hline \multicolumn{7}{|c|}{$\begin{array}{l}\text { Heterogeneity: } \text { Tau }^{2}=0.16 ; \mathrm{Chi}^{2}=3.79, \mathrm{df}=2(\mathrm{P}=0.15) ; \mathrm{I}^{2}=47 \% \\
\text { Test for overall effect: } Z=2.30(P=0.02)\end{array}$} \\
\hline \multicolumn{7}{|c|}{ 1.10.2 Allocation concealment adequate } \\
\hline EORTC-GELA H9-F & -1.2925 & 0.9426 & $4.9 \%$ & $0.27[0.04,1.74]$ & & \\
\hline $\begin{array}{l}\text { MSKCC trial \#90-44 } \\
\text { Subtotal }(95 \% \text { Cl) }\end{array}$ & -1.1671 & 0.6667 & $\begin{array}{r}9.7 \% \\
14.6 \%\end{array}$ & $\begin{array}{r}0.31[0.08,1.15] \\
\mathbf{0 . 3 0}[\mathbf{0 . 1 0}, \mathbf{0 . 8 7}]\end{array}$ & & \\
\hline \multicolumn{7}{|c|}{$\begin{array}{l}\text { Heterogeneity: } \mathrm{Tau}^{2}=0.00 ; \mathrm{Chi}^{2}=0.01, \mathrm{df}=1(\mathrm{P}=0.91) ; \mathrm{I}^{2}=0 \% \\
\text { Test for overall effect: } Z=2.22(\mathrm{P}=0.03)\end{array}$} \\
\hline Total $(95 \% \mathrm{Cl})$ & & & $100.0 \%$ & $0.40[0.27,0.61]$ & & \\
\hline \multicolumn{5}{|c|}{$\begin{array}{l}\text { Heterogeneity: } \operatorname{Tau}^{2}=0.01 ; \mathrm{Chi}^{2}=4.14, \mathrm{df}=4(\mathrm{P}=0.39) ; \mathrm{I}^{2}=3 \% \\
\text { Test for overall effect: } Z=4.33(\mathrm{P}<0.0001)\end{array}$} & $\begin{array}{|cc|}0.01 & 0.1 \\
& 1 \\
\text { Favours CMT }\end{array}$ & $\begin{array}{cc}10 & 100 \\
\text { Favours CT-alone }\end{array}$ \\
\hline
\end{tabular}

Figure 13. Forest plot of comparison: I Overall Survival, outcome: I.I 0 ITT-analysis.

\begin{tabular}{|c|c|c|c|c|c|c|}
\hline \multirow{2}{*}{$\frac{\text { Stucty or Subgroup }}{1.11 .1 \text { ITT-analysis }}$} & \multirow[t]{2}{*}{$\log [$ Hazard Ratio] } & \multirow[t]{2}{*}{ SE } & Weight & \multirow[t]{2}{*}{$\begin{array}{l}\text { Hazard Ratio } \\
\text { N, Random, } 95 \% \mathrm{Cl}\end{array}$} & \multicolumn{2}{|c|}{$\begin{array}{c}\text { Hazard Ratio } \\
\text { N, Random, 95\% } \mathrm{Cl}\end{array}$} \\
\hline & & & & & & \\
\hline EORTC-GELA H9-F & -1.2925 & 0.9426 & $4.9 \%$ & $0.27[0.04,1.74]$ & & \\
\hline MSKCC trial \#90-44 & -1.1671 & 0.6667 & $9.7 \%$ & $0.31[0.08,1.15]$ & & \\
\hline Subtotal (95唝 Cl) & & & $14.6 \%$ & $0.30[0.10,0.87]$ & & \\
\hline \multicolumn{7}{|c|}{$\begin{array}{l}\text { Heterogeneity: } \text { Tau }^{2}=0.00 ; \mathrm{Chi}^{2}=0.01, \mathrm{df}=1(\mathrm{P}=0.91) ; \mathrm{I}^{2}=0 \% \\
\text { Test for overall effect: } Z=2.22(\mathrm{P}=0.03)\end{array}$} \\
\hline \multicolumn{7}{|l|}{ 1.11.2 No ITT-analysis } \\
\hline CALGB 7751 & -0.47 & 0.9 & $5.4 \%$ & $0.63[0.11,3.65]$ & & \\
\hline GATLA 9-H-77 & -0.3484 & 0.3651 & $31.0 \%$ & $0.71[0.35,1.44]$ & -4 & - \\
\hline $\begin{array}{l}\text { Mexico B2H031 } \\
\text { Subtotal (95\% } \mathrm{Cl})\end{array}$ & -1.225 & 0.2852 & $\begin{array}{l}48.9 \% \\
85.4 \%\end{array}$ & $\begin{array}{r}0.29[0.17,0.51] \\
\mathbf{0 . 4 6}[\mathbf{0 . 2 3}, \mathbf{0 . 8 9}]\end{array}$ & & \\
\hline \multicolumn{7}{|c|}{$\begin{array}{l}\text { Heterogeneity: } \text { Tau}^{2}=0.16 ; \mathrm{Chi}^{2}=3.79, \mathrm{df}=2(\mathrm{P}=0.15) ; \mathrm{I}^{2}=47 \% \\
\text { Test for overall effect: } Z=2.30(\mathrm{P}=0.02)\end{array}$} \\
\hline Total (95\% Cl) & & & $100.0 \%$ & $0.40[0.27,0.61]$ & & \\
\hline $\begin{array}{l}\text { Heterogeneity: } \operatorname{Tau}^{2}= \\
\text { Test for overall effect }\end{array}$ & $\begin{array}{l}0.01 ; \mathrm{Chi}^{2}=4.14, \mathrm{df}= \\
\mathrm{Z}=4.33(\mathrm{P}<0.0001)\end{array}$ & $=4(\mathrm{P}=0$ & $.39) ; 1^{2}=$ & & \begin{tabular}{lc|c|c|}
0.01 & 0.1 & 1 \\
Favours CMT
\end{tabular} & \begin{tabular}{|c|c|}
10 & 100 \\
Favours CT-alone
\end{tabular} \\
\hline
\end{tabular}


Table 4. Effects of censoring on estimates for overall survival hazard ratios calculated from survival curves

\begin{tabular}{|c|c|c|}
\hline Censoring assumption & Mexico B2H031 & EORTC-GELA H9-F \\
\hline Parmar Method without corrections & $0.29[0.17$ to 0.51$]$ & $0.27[0.04$ to 1.74$]$ \\
\hline Parmar method with censoring $* 2$ & $0.27[0.16$ to 0.48$]$ & $0.16[0.02$ to 1.49$]$ \\
\hline Parmar method with censoring * 4 & $0.27[0.15$ to 0.48$]$ & $0.09[0.01$ to 1.67$]$ \\
\hline $\begin{array}{l}\text { Parmar method with censoring } * 4 \text {, assum- } \\
\text { ing censoring began at the beginning of the } \\
\text { trial }\end{array}$ & $0.27[0.15$ to 0.50$]$ & $0.09[0.00$ to 2.21$]$ \\
\hline \multicolumn{3}{|c|}{$\begin{array}{l}\text { Please note that using these methods censoring was assumed to be non-informative and constant. For the Mexico trial, even higher } \\
\text { censoring or assuming twice the amount of censoring in one of the groups had little effects on the HR. For the EORTC-GELA trial, } \\
\text { differences in censoring are expected between the two arms of the trial due to the early stopping of the arm without radiotherapy. } \\
\text { Estimating differences in median follow-up and incorporating them into the Parmar Method yielded a HR of } 0.26 \text { [0.05 to 1.72]. }\end{array}$} \\
\hline
\end{tabular}

The sensitivity analysis that included trials not fulfilling the inclusion criteria of our review protocol and including improper estimates yields a HR of 0.60 ( $95 \%$ CI 0.35 to 1.03$)$ with high heterogeneity $\left(\mathrm{I}^{2}=46 \%\right)$ Figure 14 . When excluding the trials where the number of cycles varies, the HR was 0.46 (0.27 to 0.78$)$. The two trials that examined chemotherapy plus radiotherapy versus more chemotherapy reported conflicting results among the two trials (Kung 2006; Meyer 2005). The trial by Meyer 2005 comparing two to four cycles of ABVD plus subtotal nodal irradiation with six cycles of ABVD had an (estimated) HR of 1.73 (95\% CI 0.62 to 4.86), while the Kung 2006 trial comparing four MOPP/ ABVD + IF-RT versus six MOPP/ABVD had an estimated HR of 0.86 (95\% CI 0.29 to 2.54 ). 
Figure 14. Overall Survival: Sensitivity analysis including additional trials. No early stage patients died in the Nachman 2002 and the Picardi 2007 trial.

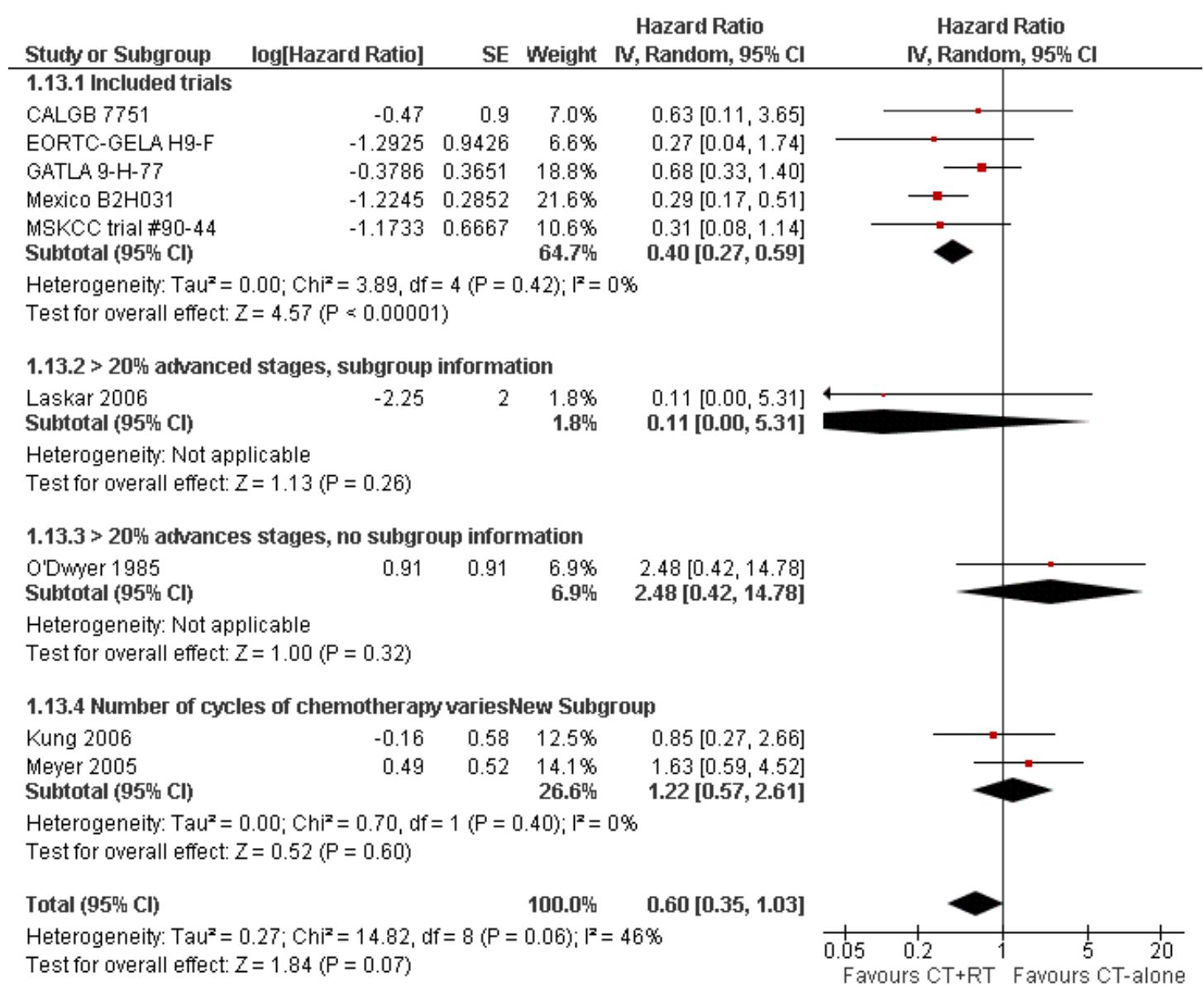

Numbers needed to treat to benefit have been calculated and are presented in Table 5 based on the main analysis.

Table 5. Number Needed To Treat To Benefit

\begin{tabular}{l|l|l}
\hline Outcome & Assumed five-year survival in the control group & NNT (95\% confidence interval) \\
\hline Tumour control & $70 \%$ & $6(5$ to 11$)$ \\
\hline Overall survival & $85 \%$ & 11 (9 to 18$)$ \\
\hline Overall survival & $97 \%$ & $55(46$ to 86$)$ \\
\hline
\end{tabular}

\section{Secondary outcomes}

\section{Tumour control}

No trial reported progression-free survival (PFS) according to the definition in the protocol (time to progression or death from any 
cause). However, four trials in the main analysis reported some progression endpoint, such as event-free survival, time to treatment failure and time to progression and were evaluated as tumour control. Exact definitions are given in Table 3. Tumour control was statistically significant in three of the four trials in which it was reported. The combination of chemotherapy and radiotherapy improved tumour control with a hazard ratio (HR) of 0.41 ( $95 \%$ confidence interval (CI) 0.25 to 0.66 , random-effects model; Figure 15). There was clear statistical heterogeneity between trials $\left(\mathrm{I}^{2}=68 \%\right)$ which may in part be due to the different definitions used. For example, some trials examined progression or freedom from treatment failure in all patients, while others examined disease free survival which is restricted to patients who reached CR. A subgroup analysis by the type of tumour control definition was statistically significant $(P=0.01$; see Figure 16$)$. The subgroup by proportion of patients with early favourable dis- ease was statistically significant $(\mathrm{P}=0.01)$. However this result is not plausible, as the group with a mixed patient population showed less effect of the addition of radiotherapy and the two groups with only early favourable and only early unfavourable patients were similar (Figure 17). The other subgroup and sensitivity analyses showed neither statistically significant difference between subgroups nor resulted in a relevant reduction of statistical heterogeneity. Factors analysed included type of chemotherapy $(\mathrm{P}=$ $0.10)$, early favourable versus unfavourable stages $(P=0.01)$, type $(\mathrm{P}=0.09)$ and timing $(\mathrm{P}=0.57)$ of radiation, and the use of quality measures (Figure 18, Figure 19, Figure 20, Figure 21, Figure 22, Figure 23). The data extraction was hampered by discrepancies in $P$ values MSKCC trial \#90-44 or number of patients included in the analysis MSKCC trial \#90-44, as well as by extraction of hazard ratios from survival curves MSKCC trial \#90-44; EORTC-GELA H9-F.

Figure 15. Forest plot of comparison: 2 Progression-Free Survival, outcome: 2.I All trials.

\begin{tabular}{|c|c|c|c|c|}
\hline \multirow{2}{*}{$\begin{array}{l}\text { Study or Subgroup } \\
\text { EORTC-GELA H9-F }\end{array}$} & \multicolumn{2}{|r|}{$\begin{array}{c}\text { Hazard Ratio } \\
\text { N, Random, 95\% } \mathrm{Cl}\end{array}$} & \multicolumn{2}{|c|}{$\begin{array}{c}\text { Hazard Ratio } \\
\text { N, Random, 95\% } \mathrm{Cl}\end{array}$} \\
\hline & $27.6 \%$ & $0.27[0.17,0.43]$ & $\longrightarrow$ & \\
\hline GATLA 9-H-77 & $28.2 \%$ & $0.55[0.35,0.86]$ & & \\
\hline Mexico B2H031 & $26.4 \%$ & $0.29[0.17,0.48]$ & & \\
\hline MSKCC trial \#90-44 & $17.8 \%$ & $0.85[0.37,1.94]$ & & \\
\hline Total $(95 \% \mathrm{Cl})$ & $100.0 \%$ & $0.41[0.25,0.66]$ & & \\
\hline \multicolumn{3}{|c|}{$\begin{array}{l}\text { Heterogeneity: } \operatorname{Tau}^{2}=0.16 ; \mathrm{Chi}^{2}=9.47, \mathrm{df}=3(\mathrm{P}=0.02) ; \mathrm{I}^{2}=68 \% \\
\text { Test for overall effect: } Z=3.64(\mathrm{P}=0.0003)\end{array}$} & $\begin{array}{lcc} & 1 & 1 \\
0.1 & 0.2 & 0.5 \\
& \text { Favours CMT }\end{array}$ & \begin{tabular}{|ccc}
2 & 5 & 10 \\
Favours & CT-alone
\end{tabular} \\
\hline
\end{tabular}


Figure 16. Forest plot of comparison: 2 Progression-Free Survival, outcome: 2.4 Definition of progression.

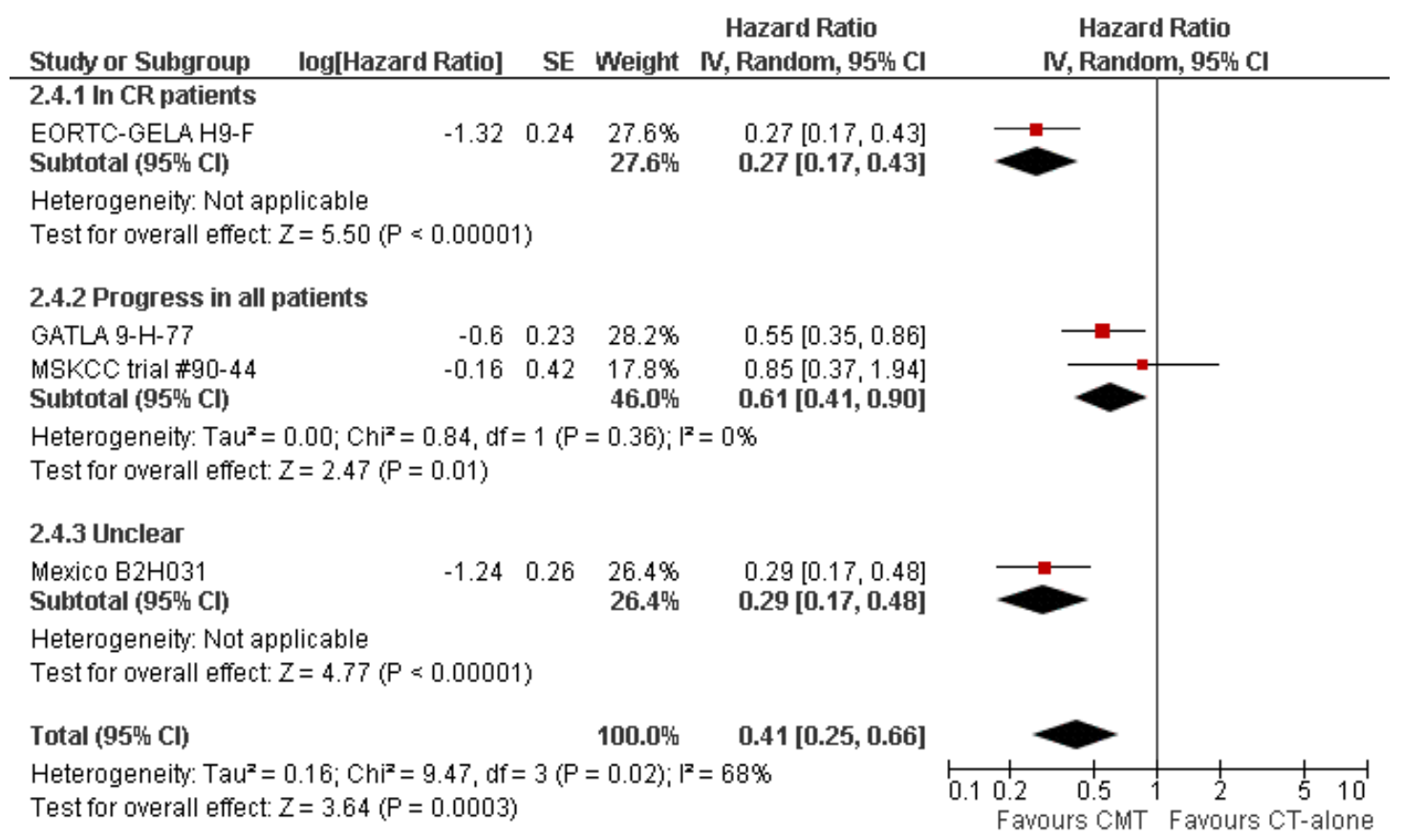

Figure 17. Forest plot of comparison: 2 Progression-Free Survival, outcome: 2.3 Proportion of patients early favourable.

\begin{tabular}{|c|c|c|c|c|c|c|c|c|}
\hline & log[Hazard Ratio] & SE & $\begin{array}{l}\text { CMT } \\
\text { Total }\end{array}$ & $\begin{array}{c}\text { CT-alone } \\
\text { Total }\end{array}$ & Weight & $\begin{array}{c}\text { Hazard Ratio } \\
\text { N, Random, 95\% } \mathrm{Cl}\end{array}$ & $\begin{array}{r}\text { Hazaro } \\
\text { N, Rando }\end{array}$ & $\begin{array}{l}\text { d Ratio } \\
\text { om, 95\% } \mathrm{Cl}\end{array}$ \\
\hline \multicolumn{8}{|c|}{ 2.3.1 All patients early favourable } & \\
\hline $\begin{array}{l}\text { EORTC-GELA H9-F } \\
\text { Subtotal (95\% Cl) }\end{array}$ & -1.32 & 0.24 & $\begin{array}{l}0 \\
\mathbf{0}\end{array}$ & $\begin{array}{l}0 \\
\mathbf{0}\end{array}$ & $\begin{array}{l}27.6 \% \\
27.6 \%\end{array}$ & $\begin{array}{r}0.27[0.17,0.43] \\
\mathbf{0 . 2 7}[\mathbf{0 . 1 7}, \mathbf{0 . 4 3}]\end{array}$ & & \\
\hline \multicolumn{8}{|c|}{ Heterogeneity: Not applicable } & Test for overall effect: $Z=5.50(P<0.00001)$ \\
\hline \multicolumn{9}{|c|}{ 2.3.2 mixed patient population ( $1 / 3$ to $1 / 2)$} \\
\hline GATLA 9-H-77 & -0.6 & 0.23 & 135 & 142 & $28.2 \%$ & $0.55[0.35,0.86]$ & & \\
\hline $\begin{array}{l}\text { MSKCC trial \#90-44 } \\
\text { Subtotal }(95 \% \text { Cl) }\end{array}$ & -0.16 & 0.42 & $\begin{array}{r}76 \\
211\end{array}$ & $\begin{array}{r}76 \\
218\end{array}$ & $\begin{array}{l}17.8 \% \\
46.0 \%\end{array}$ & $\begin{array}{r}0.85[0.37,1.94] \\
\mathbf{0 . 6 1}[\mathbf{0 . 4 1 , 0 . 9 0 ]}\end{array}$ & & \\
\hline \multicolumn{9}{|c|}{$\begin{array}{l}\text { Heterogeneity: } \text { Tau }^{2}=0.00 ; \mathrm{Chi}^{2}=0.84, \mathrm{df}=1(P=0.36) ; \mathrm{I}^{2}=0 \% \\
\text { Test for overall effect: } Z=2.47(P=0.01)\end{array}$} \\
\hline \multicolumn{9}{|c|}{ 2.3.3 All patients early unfavourable } \\
\hline $\begin{array}{l}\text { Mexico B2H031 } \\
\text { Subtotal }\left(95^{\circ} \% \mathrm{Cl}\right)\end{array}$ & -1.24 & 0.26 & $\begin{array}{l}0 \\
\mathbf{0}\end{array}$ & $\begin{array}{l}0 \\
\mathbf{0}\end{array}$ & $\begin{array}{l}26.4 \% \\
26.4 \%\end{array}$ & $\begin{array}{r}0.29[0.17,0.48] \\
\mathbf{0 . 2 9}[0.17, \mathbf{0 . 4 8}]\end{array}$ & & \\
\hline \multicolumn{9}{|c|}{ Heterogeneity: Not applicable } \\
\hline Total $(95 \% \mathrm{Cl})$ & & & 211 & 218 & $100.0 \%$ & $0.41[0.25,0.66]$ & & \\
\hline \multicolumn{7}{|c|}{$\begin{array}{l}\text { Heterogeneity: } \text { Tau }^{2}=0.16 ; \mathrm{Chi}^{2}=9.47, \mathrm{df}=3(P=0.02) ;\left.\right|^{2}=68 \% \\
\text { Test for overall effect: } Z=3.64(P=0.0003)\end{array}$} & $\begin{array}{lcc}1 & 1 \\
0.1 & 0.2 & 0.5 \\
\text { Favours CMT }\end{array}$ & 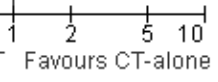 \\
\hline
\end{tabular}


Figure 18. Forest plot of comparison: 2 Progression-Free Survival, outcome: 2.2 Type of chemotherapy.

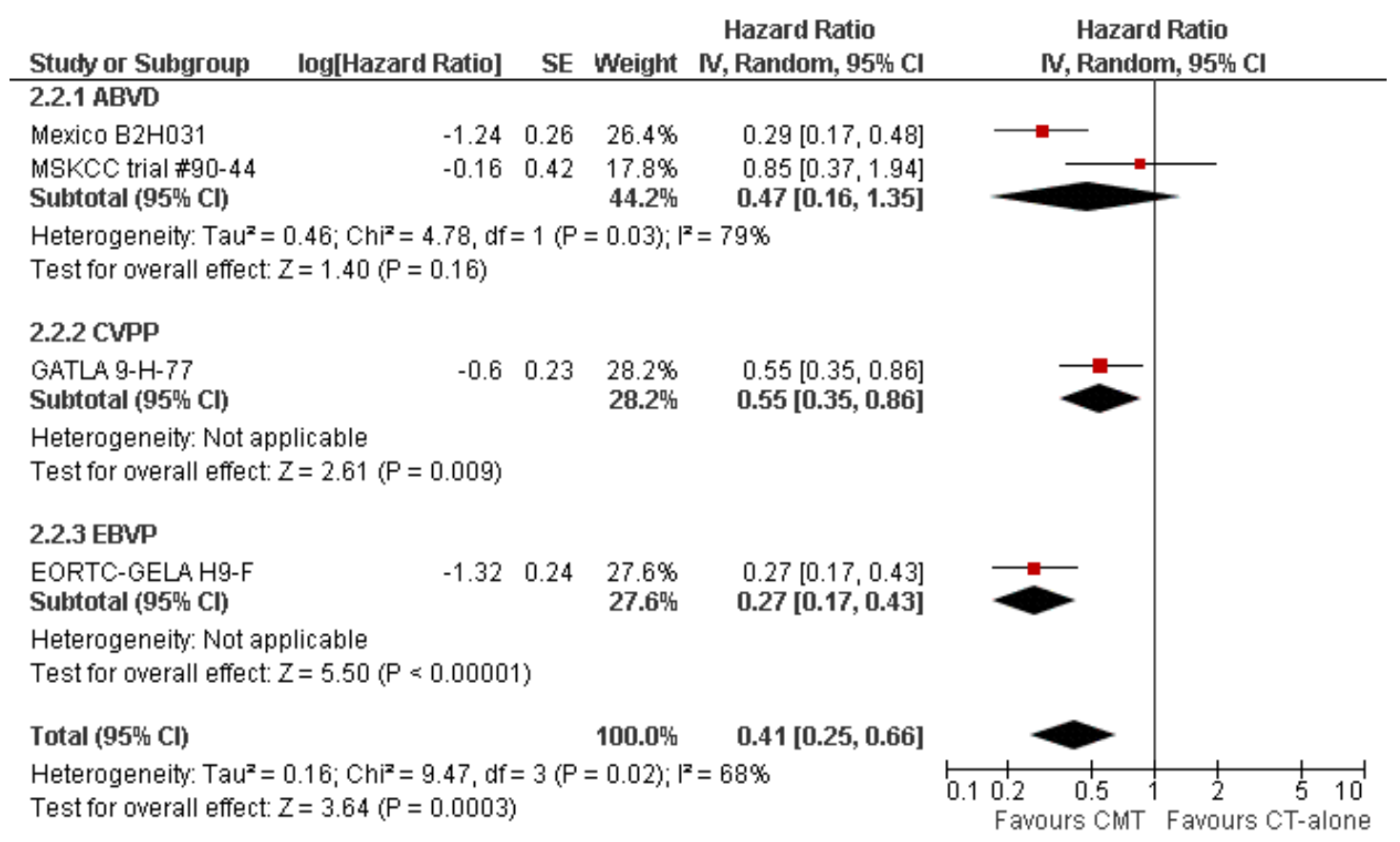

Figure 19. Forest plot of comparison: 2 Progression-Free Survival, outcome: 2.5 Timing of radiotherapy.

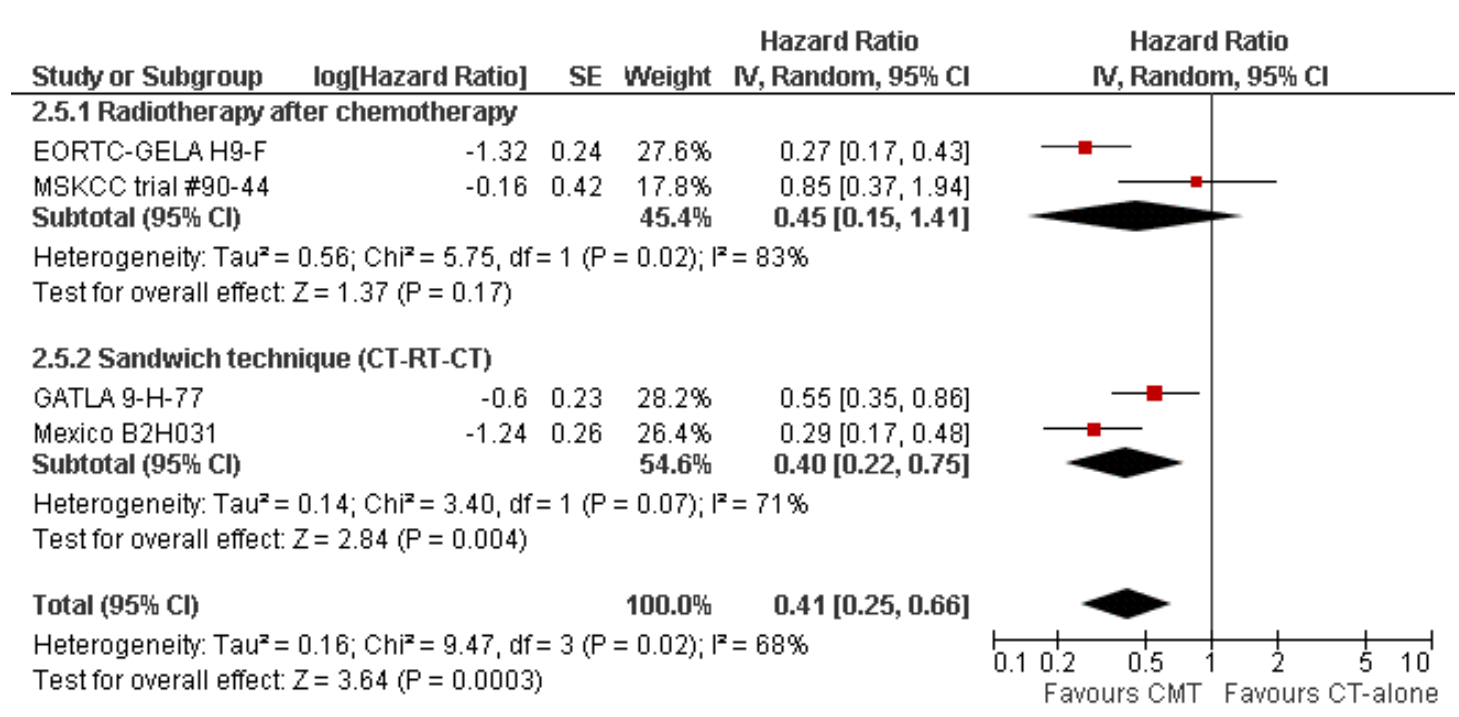


Figure 20. Forest plot of comparison: 2 Progression-Free Survival, outcome: 2.6 Type of radiotherapy.

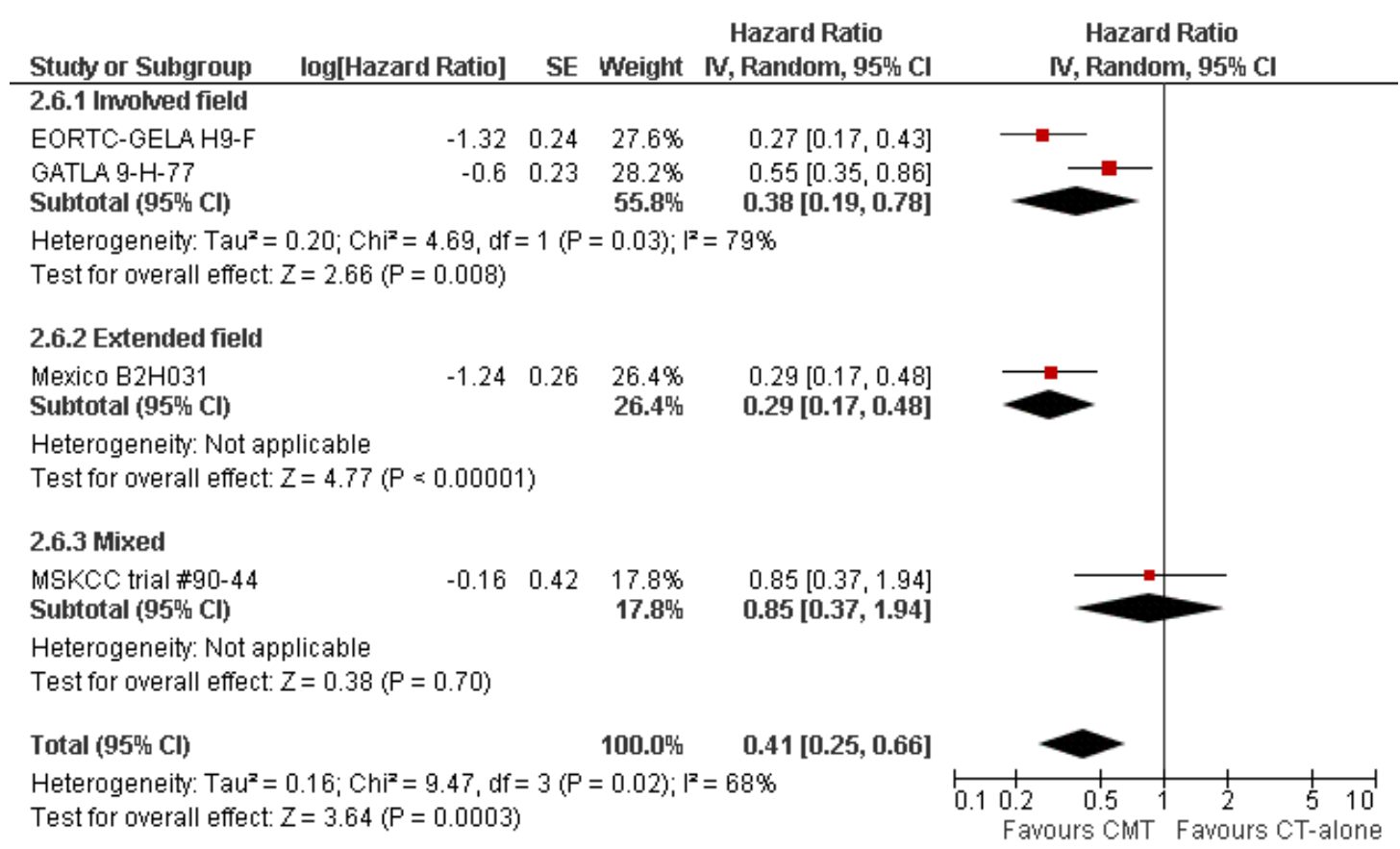

Figure 21. Forest plot of comparison: 2 Progression-Free Survival, outcome: 2.7 Length of follow-up.

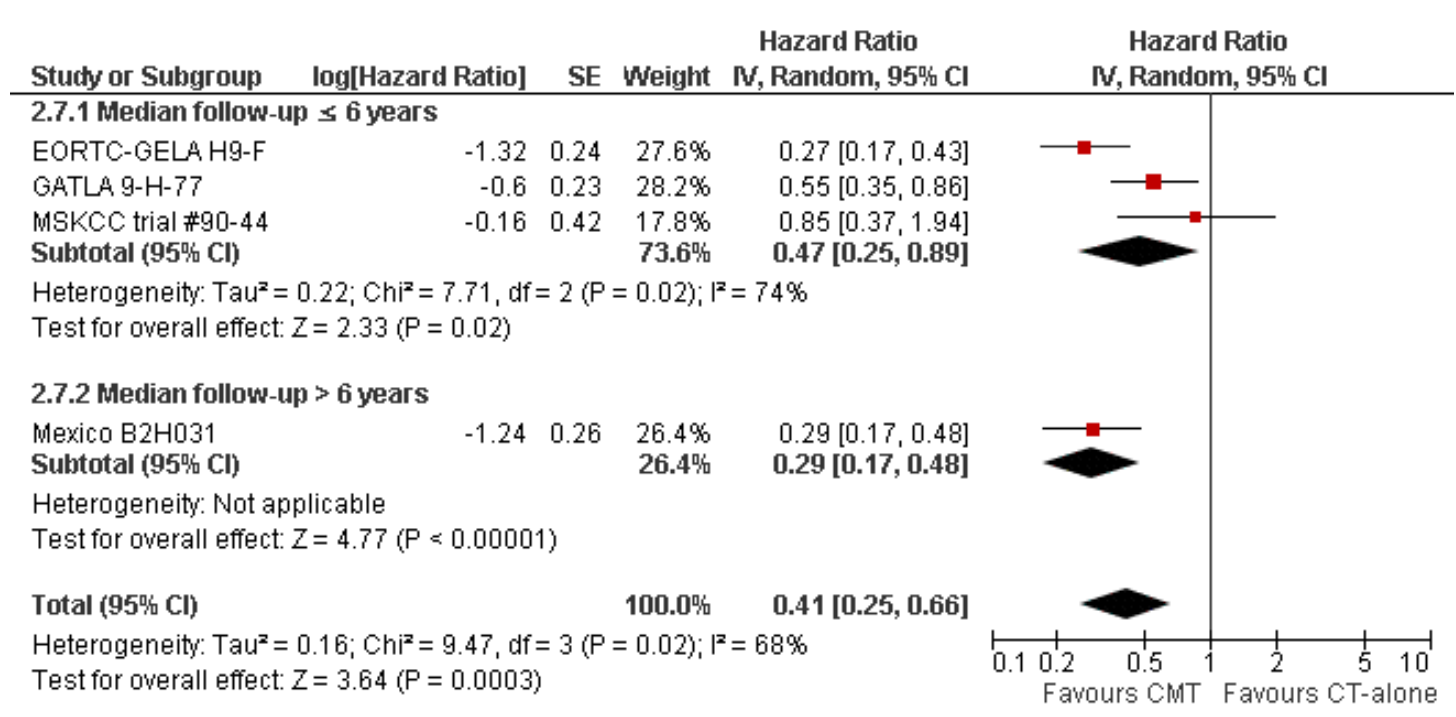


Figure 22. Forest plot of comparison: 2 Progression-Free Survival, outcome: 2.8 Allocation concealment.

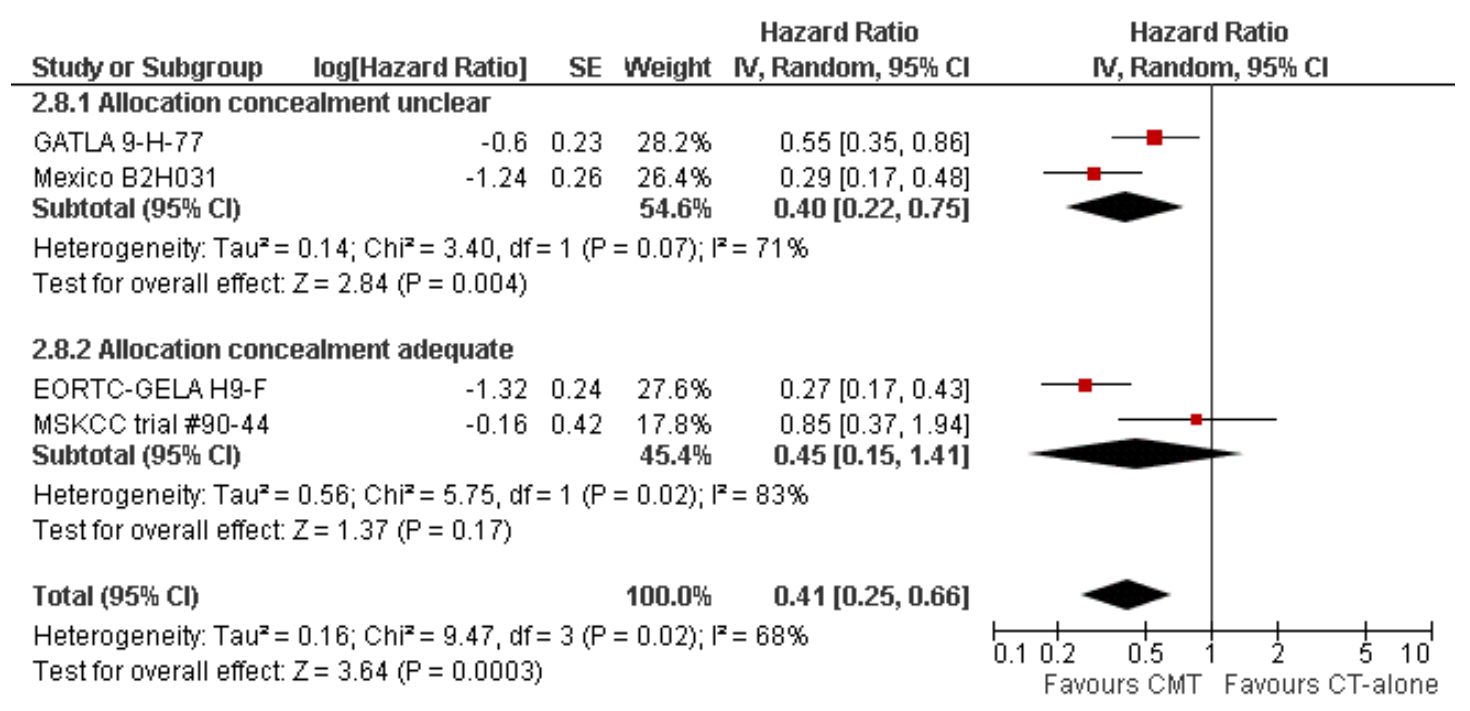

Figure 23. Forest plot of comparison: 2 Progression-Free Survival, outcome: 2.9 ITT-Analysis.

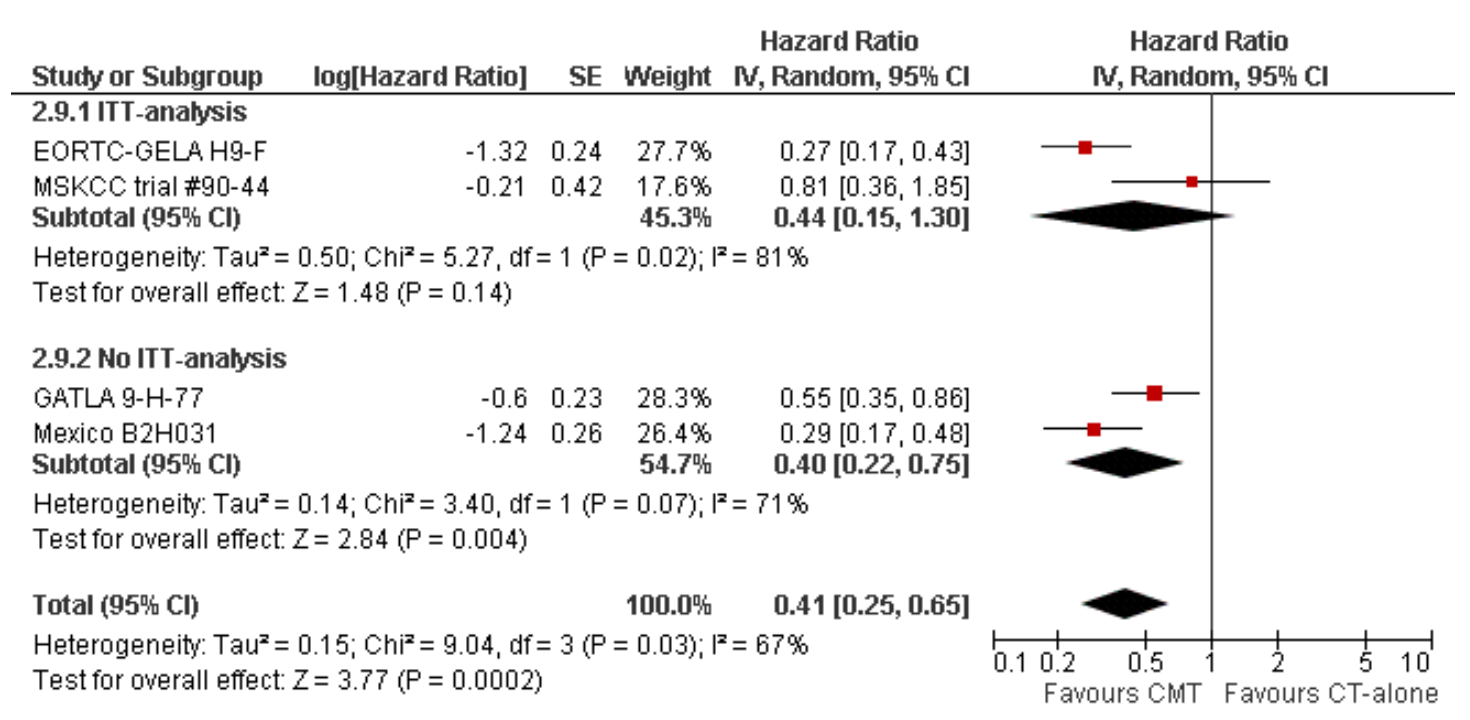

All of the trials that did not meet the strict inclusion criteria of our review protocol favoured combined modality treatment with respect to tumour control (Kung 2006; Laskar 2004; Meyer 2005; Nachman 2002; O’Dwyer 1984; Picardi 2007). When including these trials into a meta-analysis for tumour control the HR was $0.38,95 \%$ CI 0.28 to 0.57 (Figure 24). When restricting the anal- ysis to the trials that kept the number of cycles the same between the group that received radiotherapy and those that did not (without Kung 2006 and Meyer 2005), the HR was 0.39 (95\% CI 0.27 to 0.55$)$. Repeating these analyses without the improper estimates (without Nachman 2002 and Picardi 2007) yields a HR of 0.40 
(0.28 to 0.56$)$ for the full group and $0.42(0.28$ to 0.63$)$ for the analysis without Kung 2006 and Meyer 2005.

Figure 24. Tumour Control: Sensitivity analysis including additional trials. Note: improper estimates based on the number of events and the number in each group were used for the estimates of the Nachman trial and the Picardi trial. The hazard ratio without the improper estimates is $0.40(0.28$ to 0.56$)$

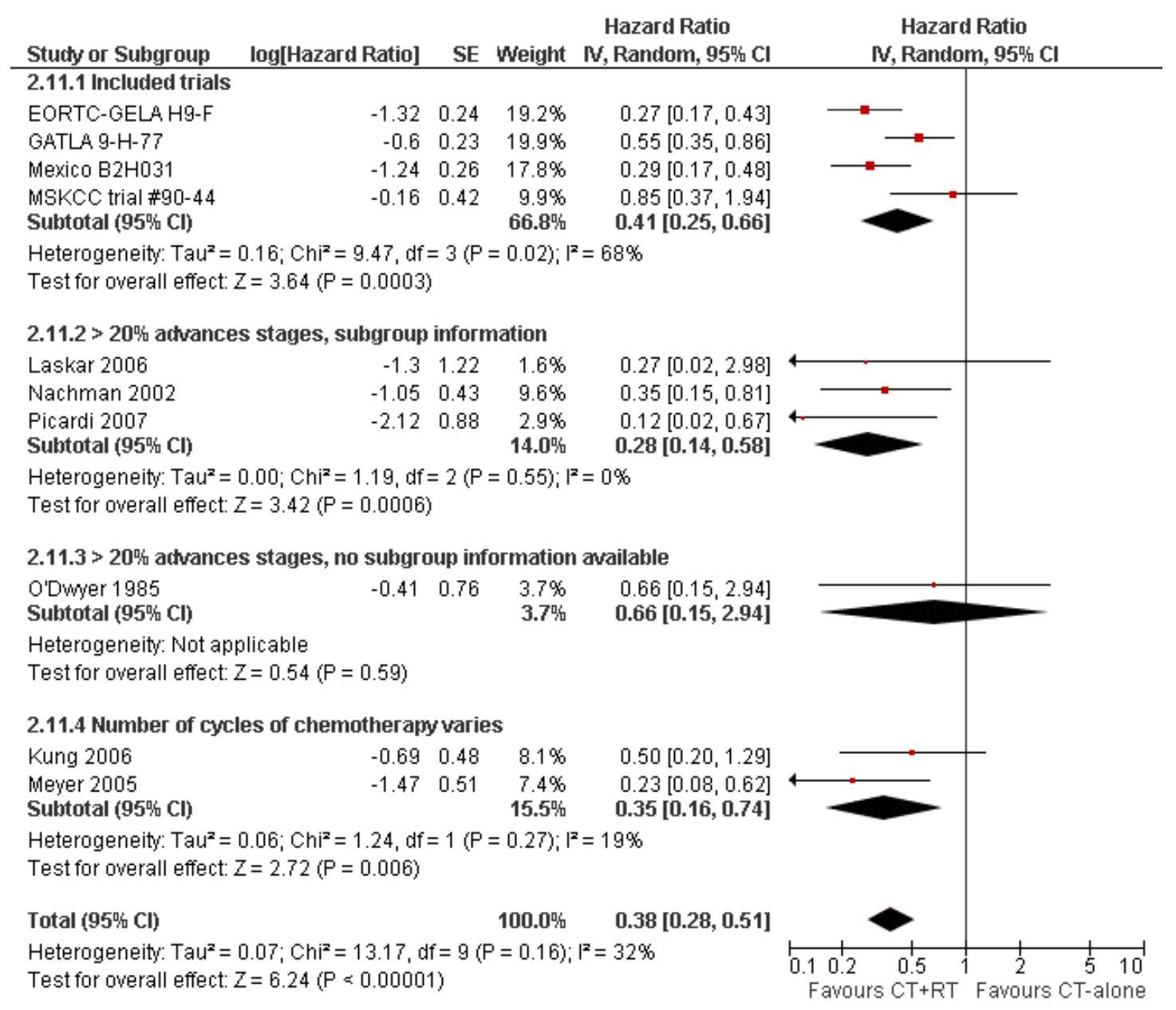

Numbers needed to treat to benefit have been calculated and are presented in Table 5 based on the main analysis.

\section{Complete response (CR)}

Four trials including 653 patients reported the $\mathrm{CR}$ rate and were meta-analysed (CALGB 7751; GATLA 9-H-77; Mexico B2H031; MSKCC trial \#90-44). No evidence of an improvement in CR in favour of CMT group was found (RR: 1.07; 95\% CI 0.98 to 1.17), using a random-effects analysis due to substantial heterogeneity ( $\mathrm{P}$ value of the homogeneity test $=0.07 ; \mathrm{I}^{2}=57 \%$ ) (Figure 25) . Subgroup analyses by chemotherapy regimen (P for subgroup differences $=0.10)$, evaluable patients $(P=0.07)$ and ITT-analysis $(P$ $=0.07$ ) were performed (Figure 26, Figure 27, Figure 28). When examining only those trials with less than $10 \%$ non-evaluable patients, the result had borderline significance $(\mathrm{HR}=1.06$; 95\% CI 1.00 to 1.12$)$ with moderate heterogeneity $\left(\mathrm{I}^{2}=20 \%\right)$ (Figure 28). 
For CR rates the sensitivity analysis including the additional trials was not performed.

Figure 25. Forest plot of comparison: 3 Complete Response Rate, outcome: 3.I All trials.

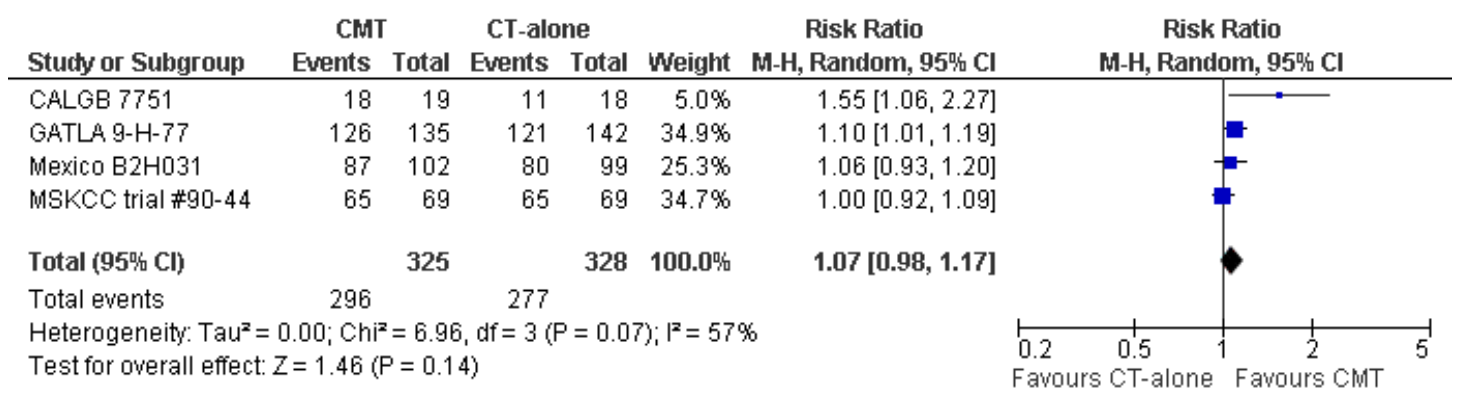

Figure 26. Forest plot of comparison: 3 Complete Response Rate, outcome: 3.2 Type of Chemotherapy.

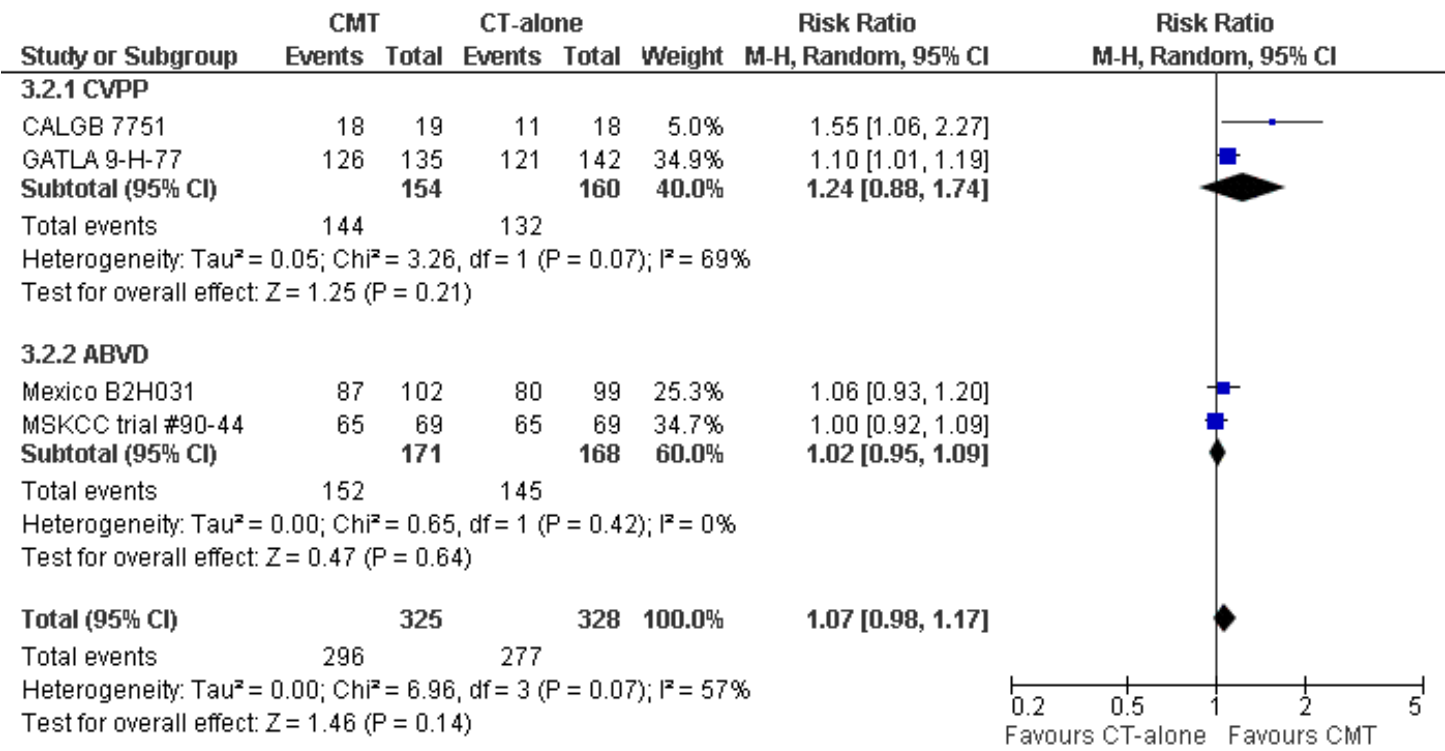


Figure 27. Forest plot of comparison: 3 Complete Response Rate, outcome: 3.3 ITT-analysis.

\begin{tabular}{|c|c|c|c|c|c|c|c|c|c|}
\hline \multirow{2}{*}{$\begin{array}{l}\text { Study or Subgroup } \\
3.3 .1 \text { ITT-analysis }\end{array}$} & \multicolumn{2}{|c|}{ CMT } & \multicolumn{2}{|c|}{ CT-alone } & \multirow[t]{2}{*}{ Weight } & $\begin{array}{c}\text { Risk Ratio } \\
\text { M-H. Random, 95吹 Cl }\end{array}$ & \multicolumn{3}{|c|}{$\begin{array}{c}\text { Risk Ratio } \\
\text { M-H, Random, } 95 \% \text { Cl }\end{array}$} \\
\hline & & & & & & & & & \\
\hline $\begin{array}{l}\text { MSKCC trial \#90-44 } \\
\text { Subtotal }(95 \% \mathrm{Cl})\end{array}$ & 65 & $\begin{array}{l}69 \\
69\end{array}$ & 65 & $\begin{array}{l}69 \\
69\end{array}$ & $\begin{array}{l}34.7 \% \\
34.7 \%\end{array}$ & $\begin{array}{l}1.00[0.92,1.09] \\
\mathbf{1 . 0 0}[\mathbf{0 . 9 2}, \mathbf{1 . 0 9}]\end{array}$ & & & \\
\hline \multicolumn{10}{|c|}{$\begin{array}{l}\text { Heterogeneity: Not applicable } \\
\text { Test for overall effect: } Z=0.00(P=1.00)\end{array}$} \\
\hline \multicolumn{10}{|l|}{ 3.3.2 no ITT-analysis } \\
\hline CALGB 7751 & 18 & 19 & 11 & 18 & $5.0 \%$ & $1.55[1.06,2.27]$ & & & \\
\hline GATLA 9-H-77 & 126 & 135 & 121 & 142 & $34.9 \%$ & $1.10[1.01,1.19]$ & & & 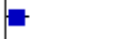 \\
\hline $\begin{array}{l}\text { Mexico B2H031 } \\
\text { Subtotal }(95 \% \mathrm{Cl})\end{array}$ & 87 & $\begin{array}{l}102 \\
256\end{array}$ & 80 & $\begin{array}{r}99 \\
259\end{array}$ & $\begin{array}{l}25.3 \% \\
65.3 \%\end{array}$ & $\begin{array}{l}1.06[0.93,1.20] \\
1.11[0.99,1.24]\end{array}$ & & & \\
\hline \multicolumn{10}{|c|}{$\begin{array}{l}\text { Heterogeneity: } \operatorname{Tau}^{2}=0.00 ; \mathrm{Chi}^{2}=3.54, \mathrm{df}=2(\mathrm{P}=0.17) ; \mathrm{I}^{2}=44 \% \\
\text { Test for overall effect: } Z=1.83(\mathrm{P}=0.07)\end{array}$} \\
\hline Total $(95 \% \mathrm{Cl})$ & & 325 & & 328 & $100.0 \%$ & $1.07[0.98,1.17]$ & & & $\gamma$ \\
\hline Total events & 296 & & 277 & & & & & & \\
\hline $\begin{array}{l}\text { Heterogeneity: } \mathrm{Tau}^{2}= \\
\text { Test for overall effect: }\end{array}$ & $\begin{array}{l}0.00 ; \mathrm{Chi}^{2} \\
\mathrm{z}=1.46(\mathrm{~F}\end{array}$ & $\begin{array}{l}=6.96 \\
=0.14\end{array}$ & 4) $d f=3(P$ & $=0.07$ & $7) ;\left.\right|^{2}=579$ & & $\begin{array}{l}0.2 \\
\text { Favo }\end{array}$ & $\begin{array}{l}0.5 \\
\text { CT-alone }\end{array}$ & Favours \\
\hline
\end{tabular}

Figure 28. Forest plot of comparison: 3 Complete Response Rate, outcome: 3.4 Number of evaluable patients.

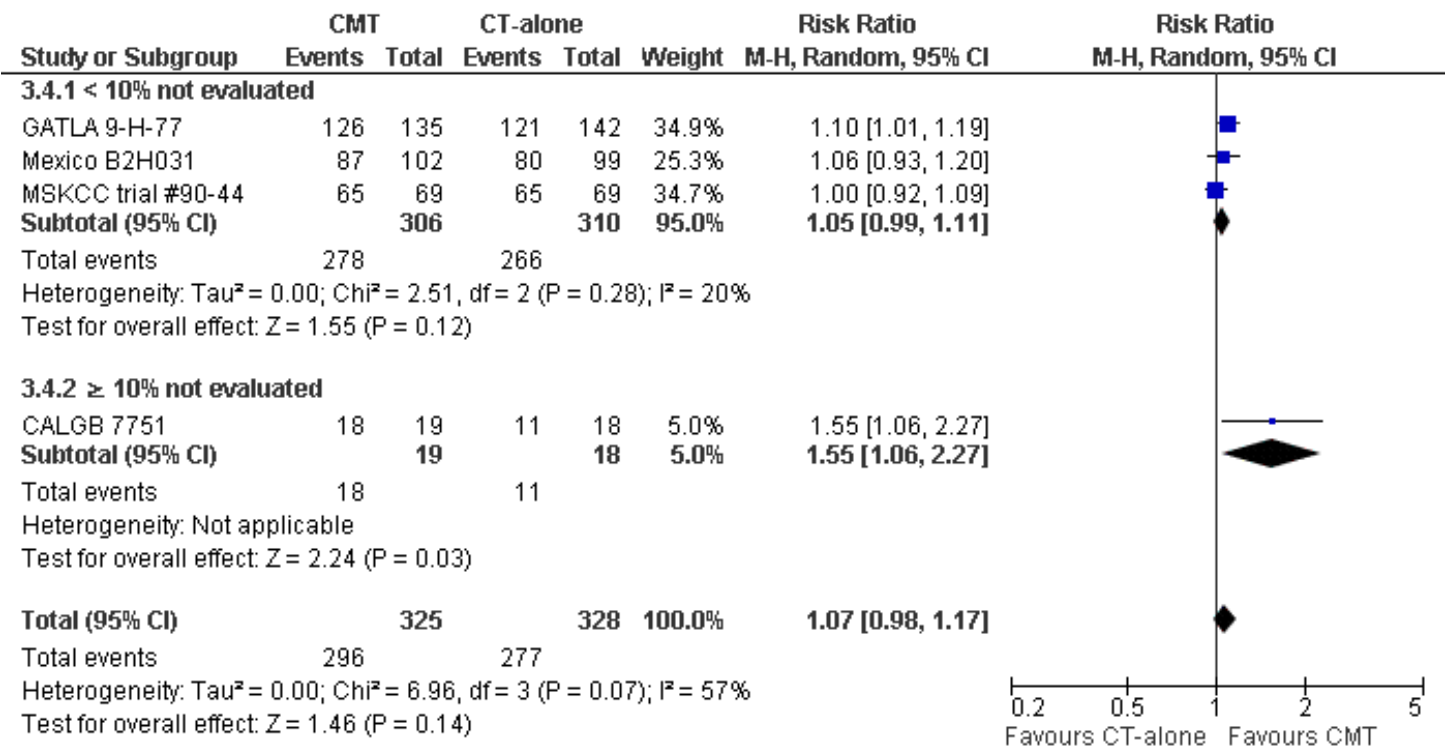




\section{Overall response rate (ORR)}

A total of 616 patients from 3 trials (GATLA 9-H-77; Mexico B2H031; MSKCC trial \#90-44) were included in the meta-analysis, of which 310 patients were in the CT-alone group and 306 patients in the CMT group. We found no evidence of a statistically significant difference regarding ORR between the CMT group and the CT-alone group (RR: 1.00; 95\% CI 0.96 to 1.06), with a fixed-effect analysis. We found no evidence of heterogeneity across the trials in the meta-analysis ( $\mathrm{P}$ value of the homogeneity test $=$ $0.68 ; I^{2}=0 \%$ ) (Figure 29).

Figure 29. Forest plot of comparison: 4 Overall Response Rate, outcome: 4.I All Trials.

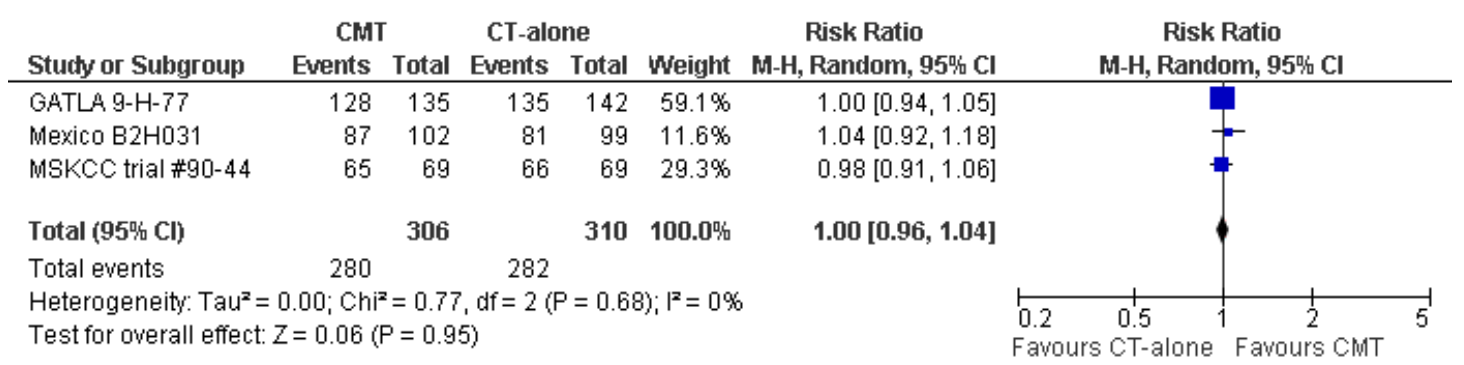

\section{Adverse events}

Adverse events, both long term toxicities and acute toxicities are tabulated in Table 6. All adverse events reported in the trials of the main analysis are in the table. Certain adverse events considered of particular interest (secondary malignancies, fertility, cardiac disease) but not reported are also listed. Most adverse events seem to be similar in both groups and are typical for the chemotherapy received (e.g. haematological effects, infections, bleomycin induced lung disease).

Table 6. Adverse Events

\begin{tabular}{|c|c|c|c|c|c|}
\hline & CALGB 7751 & $\begin{array}{l}\text { EORTC-GELA } \\
\text { H9-F }\end{array}$ & GATLA 9-H-77 & Mexico B2H031 & $\begin{array}{l}\text { MSCKK trial \#90- } \\
44\end{array}$ \\
\hline $\begin{array}{l}\text { Number of patients } \\
\text { evaluated }\end{array}$ & $\begin{array}{l}\text { 18: CT } \\
\text { 19: } \mathrm{CmT}\end{array}$ & $\begin{array}{l}\text { 130: } \mathrm{CT} \\
\text { 448: } \mathrm{CmT}\end{array}$ & $\begin{array}{l}\text { 142: } \mathrm{CT} \\
\text { 135: } \mathrm{CmT}\end{array}$ & $\begin{array}{l}\text { 99: CT } \\
\text { 102: CMT }\end{array}$ & $\begin{array}{l}\text { 76: CT } \\
\text { 76: CMT }\end{array}$ \\
\hline $\begin{array}{l}\text { Chemotherapy and } \\
\text { radiotherapy }\end{array}$ & $\begin{array}{l}6 \text { cycles of CVPP +/ } \\
\text { - involved-field ra- } \\
\text { diotherapy (dosage } \\
\text { unknown) }\end{array}$ & $\begin{array}{l}6 \text { cycles of EBVP +/ } \\
\text { - IF RT }\end{array}$ & $\begin{array}{l}6 \text { cycles of CVPP }+ \text { / } \\
\text { - IF-RT }\end{array}$ & $\begin{array}{l}6 \text { cycles of ABVD + } \\
\text { - EF-RT }\end{array}$ & $\begin{array}{l}6 \text { cycles of ABVD +/ } \\
\text { - EF or IF RT }\end{array}$ \\
\hline
\end{tabular}




\begin{tabular}{|c|c|c|c|c|c|}
\hline $\begin{array}{l}\text { Median duration of } \\
\text { follow-up }\end{array}$ & 1.8 years & 4.3 years & 4 years & 11.4 years & 5.6 years \\
\hline $\begin{array}{l}\text { Leukopenia or neu- } \\
\text { tropenia }\end{array}$ & NR & NR & $\begin{array}{l}\text { "mild" in both } \\
\text { groups } \\
\text { CT: } 12 \% \\
\text { CMT } 14 \%\end{array}$ & $\begin{array}{l}\text { CT grade I-II: } 31 \\
(31 \%) \text {; grade III-IV: } \\
7(7 \%) \\
\text { CMT grade I-II: } 39 \\
(39 \%) \text {; grade III-IV } \\
24(22 \%)\end{array}$ & $\begin{array}{l}\text { Leukope- } \\
\text { nia: } 27(18 \%) \text { de- } \\
\text { veloped grade II-IV } \\
\text { leukopenia } \\
12 \text { patients hospi- } \\
\text { talised for neutrope- } \\
\text { nia }(8 \%) \\
\text { After } \\
\text { a protocol amend- } \\
\text { ment } 1992 \text { to allow } \\
\text { the use of prophy- } \\
\text { lactic filgrastim, up } \\
\text { to } 62 \% \text { of patients } \\
\text { received filgrastim }\end{array}$ \\
\hline $\begin{array}{l}\text { Infections and in- } \\
\text { fection-related mor- } \\
\text { tality }\end{array}$ & NR & NR & $\begin{array}{l}\text { CT: } 1 \text { patient died } \\
\text { of sepsis } \\
\text { CMT: } 2 \text { patients } \\
\text { died of sepsis }\end{array}$ & $\begin{array}{l}\text { no febrile neutrope- } \\
\text { nia was observed }\end{array}$ & $\begin{array}{l}1 \text { patient died of } \\
\text { sepsis and pneumo- } \\
\text { nia in the context of } \\
\text { neutropenia }\end{array}$ \\
\hline Thrombopenia & NR & NR & NR & $\begin{array}{l}\text { CT: } 2(2 \%) \\
\text { CMT: } 5(4 \%) \\
\text { Grades I-II }\end{array}$ & $\begin{array}{l}\text { Grade } \\
4 \text { thrombopenia in } 3 \\
\text { patients }(2 \%)\end{array}$ \\
\hline Anaemia & NR & NR & NR & NR & $\begin{array}{l}\text { Grade } 3 \text { decrease in } \\
\text { haemoglobin in } 5 \\
\text { patients }(3 \%) \text {, grade } \\
4 \text { decrease in } 2 \text { pa- } \\
\text { tients }(1 \%)\end{array}$ \\
\hline Neurotoxicity & NR & NR & $\begin{array}{l}\text { "no severe case was } \\
\text { reported" }\end{array}$ & $\begin{array}{l}\text { CT: grade I-II } 24 \\
(24 \%) \\
\text { CMT grade I-II } 36 \\
(35 \%) \text {; grade III-IV } \\
3(3 \%)\end{array}$ & NR \\
\hline Alopecia & NR & NR & "mild" & $\begin{array}{l}\text { CT: } 34(34 \%) \\
\text { CMT: } 29(28 \%) \\
\text { Grade III-IV } \\
\text { CT: } 13(13 \%) \\
\text { CMT: } 8(8 \%)\end{array}$ & NR \\
\hline $\begin{array}{l}\text { Nausea and Vomit- } \\
\text { ing }\end{array}$ & NR & NR & "mild" & $\begin{array}{l}\text { CT grade I-II: } 46 \\
(46 \%) \\
\text { CMT grade I-II: } 63\end{array}$ & $\begin{array}{l}\text { Grade } 3 \text { nausea in } \\
15 \text { patients }(10 \%) \\
\text { Grade } 3 \text { emesis in } 7\end{array}$ \\
\hline
\end{tabular}


Table 6. Adverse Events (Continued)

\begin{tabular}{|c|c|c|c|c|c|}
\hline & & & & $(61 \%)$ & patients $(5 \%)$ \\
\hline Lung fibrosis & NR & NR & NR & 0 patients & $\begin{array}{l}33 \text { pa- } \\
\text { tients (22\%) (18 pa- } \\
\text { tients ABVD+RT, } \\
15 \text { ABVD- } \\
\text { alone) discontinued } \\
\text { bleomycin due to an } \\
\text { in- } \\
\text { crease of more than } \\
20 \% \text { in the DLCO } \\
\text { (diffusion lung ca- } \\
\text { pacity) test; } 10 \text { pa- } \\
\text { tients were treated } \\
\text { with glycocor- } \\
\text { ticoids. None devel- } \\
\text { oped chronic symp- } \\
\text { tomatic pulmonary } \\
\text { disease. One patient } \\
\text { died of bleomycin } \\
\text { induced toxicity. }\end{array}$ \\
\hline $\begin{array}{l}\text { Secondary } \\
\text { malignancy }\end{array}$ & NR & $\begin{array}{l}1 \text { patient died of } \\
\text { AML (number of } \\
\text { secondary } \\
\text { malignancies not re- } \\
\text { ported) }\end{array}$ & $\begin{array}{l}\text { One patient in the } \\
\text { CT group devel- } \\
\text { oped bladder can- } \\
\text { cer. }\end{array}$ & $\begin{array}{l}\text { CT: } 2 \text { patients de- } \\
\text { veloped malignant } \\
\text { lymphoma } 3.6 ; 6.4 \\
\text { years after comple- } \\
\text { tion of treatment } \\
\text { (both alive at the } \\
\text { end of study) } \\
\text { CMT: } 2 \text { patients } \\
\text { had MDS and sub- } \\
\text { sequent acute } \\
\text { leukaemia. } \\
\text { They died } 3.1 \text { and } \\
9.5 \text { years after com- } \\
\text { pletion of therapy }\end{array}$ & $\begin{array}{l}\text { CT: } 2 \text { patients with } \\
\text { secondary ma- } \\
\text { lignancies observed } \\
\text { (gastric carcinoma, } \\
2 \text { non-fatal skin can- } \\
\text { cers in one patient) } \\
\text { CMT: } 5 \text { secondary } \\
\text { malignancies: } \\
3 \text { prior to RT: fol- } \\
\text { licular thyroid car- } \\
\text { cinoma, } 2 \text { patients } \\
\text { with follicular B-cell } \\
\text { non-Hodgkin's } \\
\text { lymphoma; } 2 \text { after } \\
\text { RT: one papillary } \\
\text { thyroid carcinoma, } \\
\text { one parotid carci- } \\
\text { noma }\end{array}$ \\
\hline Infertility & NR & NR & NR & NR & NR \\
\hline Cardiac disease & $\begin{array}{l}1 \text { patient in the } \\
\text { CMT group died of } \\
\text { a heart attack during } \\
\text { chemotherapy }\end{array}$ & NR & NR & NR & NR \\
\hline
\end{tabular}




\section{DISCUSSION}

The following findings emerge from this meta-analysis:

1. In patients with early stage HL, there is no evidence that the complete response rate is different in patients receiving chemotherapy alone compared to those receiving combined modality treatment (CMT) consisting of chemotherapy plus radiotherapy.

2. Tumour control is better in patients receiving CMT compared to chemotherapy alone.

3. Adding radiotherapy to chemotherapy improves overall survival in this group of patients.

To our knowledge this is the first comprehensive review focusing on patients with early stage Hodgkin lymphoma that compares chemotherapy alone with CMT consisting of chemotherapy plus additional radiotherapy. The main analysis according to the strict inclusion criteria of our review protocol included five randomised controlled trials with 1245 patients of both early favourable and early unfavourable Hodgkin lymphoma. The literature search revealed a number of trials with more than $20 \%$ of patients in advanced stages or with fewer cycles of chemotherapy in patients receiving radiotherapy. Because these trials were considered to be relevant to the underlying clinical question, these trials were included in sensitivity analyses, where subgroup information of early stage patients was used if available. These sensitivity analyses underlined the results of the main analyses.

The hazard ratio for overall survival $(0.40 ; 95 \%$ CI 0.27 to 0.61$)$ is similar to that of tumour control. With an assumed OS of $85 \%$ at five years as observed in the three trials included here (GATLA 9-H-77; Mexico B2H031; MSKCC trial \#90-44), approximately 11 patients (95\% CI 9 to 18 ) would require treatment with CMT for one additional patient to survive during the first five years. With better survival (97.5\%) as reported in the EORTC-GELA H9-F trial, approximately 55 patients (95\% CI 46 to 86) would be needed. (For other numbers needed to treat to benefit see Table 5). These potentially surprising results were robust when excluding the Mexico B2H031 trial, which had a high weight in the analysis due to the long period of follow-up and the relatively high mortality. The only concern in the overall survival analyses stems from hazard ratios estimated from survival curves (EORTC-GELA H9-F; Mexico B2H031) and the use of preliminary data from the EORTC-GELA H9-F trial. However, these are minor points, as the direction of the effect is clear and only the magnitude may vary slightly. Again, the sensitivity analysis including additional trials (Kung 2006; Laskar 2004; Meyer 2005; Nachman 2002; O’Dwyer 1985; Picardi 2007) also supported the results in favour of CMT. Two additional trials had effect estimates favouring CMT, two trials observed no deaths (Nachman 2002; Picardi 2007) and two trials observed a slight effect favouring chemotherapy alone, which was not statistically significant. One of these trials is a very small, very old trial using MOPP chemotherapy (O’Dwyer 1985). The other trial Meyer 2005, similar to the trial by Kung 2006 replaced two to four cycles of ABVD chemotherapy with subtotal nodal irradiation. They are therefore not directly comparable to the trials which used the same number of cycles in both arms of the trials, nor to trials examining smaller radiation fields.

In the present analyses we found no evidence for interaction of treatment options or patient characteristics. Two of five trials employed ABVD, and the summary hazard ratios in the subgroup analyses by chemotherapy regimen were very similar. No difference in tumour control or OS was observed between trials that examined the addition of involved field or extended field radiotherapy. This is in line with the finding that extended field radiotherapy had little or no advantage over involved field radiotherapy in Hodgkin patients when used alone or after effective chemotherapy (Engert 2003; Franklin 2005; Specht 1998). In addition, subgroup analyses gave no hint that the hazard ratios may differ depending on the proportion of patients with early favourable or early unfavourable disease or the inclusion or exclusion of patients with bulky disease. However, due to the small number of trials included, obtaining reliable information from subgroup analyses is unlikely.

Long term adverse effects such as secondary malignancies or cardiac disease are important in HL patients and can occur later than the reported observation times of the discussed trials. Radiotherapy can induce secondary malignancies while the exact increase in the risk is unclear. The absolute risk difference between chemotherapy alone and chemotherapy plus radiotherapy in a recent individual patient data meta-analysis was about $1 \%$ in early stage patients at 15 years (Franklin 2005). Some cohorts of Hodgkin patients of any stage suggest that this difference may be higher (Bhatia 2003; Dores 2002; Swerdlow 2003; van Leeuwen 2000). Unfortunately, the results of these cohorts are mixed and far from clear (see Table 7). In addition, patients who relapse have a more pronounced risk of secondary malignancies according to a cohort study by Aleman 2003. Therefore the addition of radiotherapy which reduces the number of patients with relapse (who require more aggressive therapy) may contribute to a smaller overall risk difference than suggested by the cohorts who do not distinguish between relapsed an non-relapsed patients when evaluating the risk of radiotherapy. 
Table 7. Risk of secondary malignancies in cohorts of patients with Hodgkin lymphoma

\begin{tabular}{|c|c|c|c|c|}
\hline Cohort & Comparison & Measure & Results & notes \\
\hline Dores 2002 & $\begin{array}{l}\text { any radiotherapy vs. che- } \\
\text { motherapy alone }\end{array}$ & $\begin{array}{l}\text { observed divided by ex- } \\
\text { pected }(\mathrm{O} / \mathrm{E})\end{array}$ & $\begin{array}{l}1 \text { to } 9 \text { years after treat- } \\
\text { ment: } 488 \text { RT and } 221 \\
\text { CT pts: O/E } 2.3(\mathrm{RT}) \text { and } \\
2.3(\mathrm{CT}) \text {; } \\
\mathbf{1 0} \text { to } \mathbf{1 4} \text { years: } 206 \text { and } \\
48 \text { pts; } 3.2(\mathrm{RT}) \text { and } 2.6 \\
(\mathrm{CT}) ; \\
\mathbf{1 5} \text { to } \mathbf{1 9} \text { years: } 132 \mathrm{RT} \\
\text { and } 14 \mathrm{CT} \text { pts } \mathrm{O} / \mathrm{E}: 3.5 \\
(\mathrm{RT}) \text { and } 1.7(\mathrm{CT})\end{array}$ & no stage information \\
\hline van Leeuwen 2000 & $\begin{array}{l}\text { initial CT+ RT (first year) } \\
\text { and initial CT alone }\end{array}$ & $\begin{array}{l}\text { Relative risk compared } \\
\text { to general population by } \\
\text { type of cancer } \\
\text { CT-alone: } 75 \text { pts; CT + } \\
\text { RT: } 234 \text { pts }\end{array}$ & $\begin{array}{l}\text { breast cancer: } \\
\text { CT-alone: } \\
\text { (no cases) } \\
\text { CT + RT as initial ther- } \\
\text { apy: } 7.5 \text { ( } 2.7 \text { to } 16.3) \\
\text { All solid tumours except } \\
\text { breast: } \\
\text { CT-alone: } \\
1.3 \text { ( } 0.03 \text { to } 7.4) \\
\text { CT + RT as initial ther- } \\
\text { apy: } 4.4(2.0 \text { to } 8.3)\end{array}$ & $\begin{array}{l}\text { adolescence and } \\
\text { early adulthood; no stage } \\
\text { information; no informa- } \\
\text { tion about the length of } \\
\text { follow-up in each group }\end{array}$ \\
\hline Swerdlow 2000 & final treatment received & $\begin{array}{l}\text { standardized incidence ra- } \\
\text { tio (SIR) compared to the } \\
\text { general population by age } \\
\text { of first treatment }\end{array}$ & $\begin{array}{l}\text { Chemotherapy alone: } \\
\text { age } 25 \text { to } 44: \text { SIR }=4.0 \\
(2.7 \text { to } 5.6) \text {; age } 45 \text { to } 54: \\
\text { SIR }=2.3(1.4 \text { to } 3.4) \\
\text { CT }+ \text { RT: } \\
\text { age } 25 \text { to } 44 \text { SIR }=4.2(3.1 \\
\text { to } 5.6) ; \\
\text { age } 45 \text { to } 54 \text { SIR }=3.8(2.5 \\
\text { to } 5.4)\end{array}$ & no stage information \\
\hline Bhatia 2003 & final treatment received & $\begin{array}{l}\% \text { of pts with secondary } \\
\text { malignancies }\end{array}$ & $\begin{array}{l}\text { Chemotherapy alone: } \\
9.4 \% \text { of } 106 \text { pts; } \\
\text { CT + RT: } \\
12.7 \% \text { of } 960 \text { pts. }\end{array}$ & $\begin{array}{l}\text { no information on stage, } \\
\text { nor on original therapy, } \\
\text { nor duration of follow-up }\end{array}$ \\
\hline
\end{tabular}

Cardiac disease is associated with mediastinal radiation and with the cumulative dose of chemotherapy, in particular doxorubicin (Singal 1998). Radiation fields have been reduced substantially from extended field radiotherapy to involved field or even involved node radiotherapy used today. Replacing consolidation radiotherapy with chemotherapy, as evaluated by Meyer 2005, increases the dose of doxorubicin and may thus increase long term cardiac tox- icity. Importantly, there was also a detrimental effect on progression-free survival in the group of patients receiving chemotherapy only.

Identifying patients at low risk of relapse might be considered an alternative to consolidation radiotherapy for all patients. Currently, however, no reliable evidence for such a strategy exists. In this 
meta-analysis, the presence of conventional CR after chemotherapy such as ABVD (Mexico B2H031; MSKCC trial \#90-44) or EBVP (EORTC-GELA H9-F) does not seem to accurately identify a group of patients who do not benefit from radiotherapy with regard to tumour control. PET may be an alternative. However in patients with bulky early stage disease having residual masses in CT that are PET negative, the rate of relapse after ABVD chemotherapy was much higher in patients who did not receive consolidation radiotherapy (Picardi 2007). Two other trials are planed or under way in patients with early stage Hodgkin lymphoma (clinicaltrials.gov identifiers: NCT00736320, NCT00433433).

\section{A U T H OR S' CONCLUSIONS Implications for practice}

Adding radiotherapy to a commonly used chemotherapy regimen such as ABVD increases five-year overall survival and tumour control in patients with early stage Hodgkin lymphoma.

\section{Implications for research}

Since adding radiotherapy may result in more secondary malignancies or cardiac disease and deaths thereof, long-term follow-up (more than 15 years) of clinical trials examining treatment options in early stage HL would be helpful. In addition, clear definitions of outcomes that examine tumour control would be useful in order to reduce heterogeneity. We recommend the use of progressionfree survival, i.e. time to progression, relapse or death of any cause.

\section{ACKNOWLEDGEMENTS}

We are grateful to the following persons for their comments and improving the review:

Dr. Sue Richards and Prof. Benjamin Djulbegovic, Editors of the Cochrane Haematological Malignancies Group,

Sabine Kluge and Dr. Kathrin Bauer from Cochrane Haematological Malignancies Group.

\section{REFERENCES}

\section{References to studies included in this review}

\section{CALGB 7751 \{published data only\}}

Bloomfield CD, Pajak TF, Glicksman AS, Gottlieb AJ, Coleman M, Nissen NI, et al.Chemotherapy and combined modality therapy for Hodgkin's disease: a progress report on Cancer and Leukemia Group B studies. Cancer Treatment Reports 1982; Vol. 66, issue 4: 835-46.

EORTC-GELA H9-F \{published data only\}

Eghbali H, Brice P, Creemers GY, Kooji MM, Carde P, Van't Veer $\mathrm{MB}$, et al.Comparson of three radiation dose levels after EBVP regimen in favourable supradiaphragmatic clinical stages (CS) I-II Hodgkin's lymphoma (HL): Preliminary results of EORTC-GELA H9-F trial. Blood 2005;106(11 suppl.):abstract 814 .

Noordijk EM, Thomas J, Ferme' C, van 't Veer MB, Brice P, Divine' $\mathrm{M}$, et al.First results of EORTC-GELA $\mathrm{H}$ ) randomized trials: the $\mathrm{H} 9-\mathrm{F}$ trial (comparing 3 radiation dose levels) and H9-U trial (comparing 3 chemotherapy schemes) in patients with favourable or unfavourable early stage Hodgkin's lymphoma (HL). Journal of Clinical Oncology 2005; Vol. 23, issue suppl.:abstract 6505.

Noordijk EM, Thomas J, Fermé C, Van't Veer MB, Brice P, Divine $\mathrm{M}$, et al.First results of the EORTC-GELA H9 randomized trials: the H9-F trial (comparing 3 radiation dose levels) and H9-U trial (comparing 3 chemotherapy schemes) in patients with favorable or unfavorable early stage Hodgkin's lymphoma (HL). ASCO Annual Meeting Presentation Slides YR:2005.

Thomas J, Fermé C, Noordijk EM, Eghbali H, Henry-Amar M. The EORTC-GELA treatment strategy in clinical stages I-II HL: Results of the H9-F and H9-U trials. International Symposium on Hodgkin Lymphoma, Cologne, Presentation YR:2007.

Thomas J, Fermé C, Noordijk EM, van 't Veer MB, Brice P, Divine
M, et al.Results of the EORTC-GELA H9 randomized trials: The $\mathrm{H} 9-\mathrm{F}$ trial (comparing 3 radiation dose levels) and $\mathrm{H} 9-\mathrm{U}$ trial (comparing 3 chemotherapy schemes) in patients with favorable or unfavorable early stage Hodgkin's lymphoma (HL). Haematologica 2007;92(suppl. 5):27.

GATLA 9-H-77 \{published data only\}

Pavlovsky S, Maschio M, Santarelli MT, Muriel FS, Corrado C,

Garcia I, et al.Randomized trial of chemotherapy versus chemotherapy plus radiotherapy for stage I-II Hodgkin's disease. Journal of the National Cancer Institute 1988;80(18):1466-73.

\section{Mexico B2H031 \{published data only\}}

Aviles A, Delgado S. A prospective clinical trial comparing chemotherapy, radiotherapy and combined therapy in the treatment of early stage Hodgkin's disease with bulky disease. Clinical \& Laboratory Haematology 1998;20(2):95-9.

MSKCC trial \#90-44 \{published data only\}

Straus DJ, Portlock CS, Qin J, Myers J, Zelenetz AD, Moskowitz $\mathrm{C}$, et al.Results of a prospective randomized clinical trial of doxorubicin, bleomycin, vinblastine, and dacarbazine (ABVD) followed by radiation therapy (RT) versus ABVD alone for stages I, II, and IIIA nonbulky Hodgkin disease. Blood 2004;104(12): 3483-9.

\section{References to studies excluded from this review}

\section{Andrieu 1999 \{published data only\}}

Andrieu JM, Jais JP, Escoffre-Barbe M, Delwail V, Desablens B, Kiladjian JJ, et al.Bulky Hodgkin's disease (B-HD): treatment with an initial 7 drug chemotherapy (CT) delivered over 12 weeks followed by high dose extended field irradiation (EF-RT). Seven 
year results of the GOELAMS H90M multicentric randomized trial. Blood 1999;94(10 suppl.):abstract 528a.

Bonnet 2007 \{published data only\}

Bonnet C, Fillet G, Mounier N, Ganem G, Molina TJ, Thiéblemont C, et al.CHOP alone compared with CHOP plus radiotherapy for localized aggressive lymphoma in elderly patients: a study by the Groupe d'Etude des Lymphomes de l'Adulte. Journal of Clinical Oncology 2007;25(7):787-92.

Brusamolino 1994 \{published data only\}

Brusamolino E, Lazzarino M, Orlandi E, Canevari A, Morra E, Castelli G, et al.Early-stage Hodgkin's disease: long-term results with radiotherapy alone or combined radiotherapy and chemotherapy. Annals of Oncology 1994;5(suppl. 2):101-6.

\section{Cheveresan 1998 \{published data only\}}

Cheveresan LF, Roth I, Balan M, Ionita H. Combined modality therapy in early stage Hodgkin's disease - preliminary results of a clinical trial. Leukemia and Lymphoma 1998;29(suppl. 1).

Cimino 1990 \{published data only\}

Cimino G. Chemotherapy alone for the treatment of early-stage Hodgkin's disease. European Journal of Cancer 1990;26(11-12): $1115-8$.

\section{Cosset 1992 \{published data only\}}

Cosset JM, Henry-Amar M, Meerwaldt JH, Carde P, Noordijk EM, Thomas J, et al.The EORTC trials for limited stage Hodgkin's disease. The EORTC Lymphoma Cooperative Group. European journal of cancer 1992;28A(11):1847-50.

\section{Desablens 1999 \{published data only\}}

Desablens B, Jais JP, Lacotter-Thierry L, Foussard C, EscoffreBarbe M, Moreau P, et al.Treatment of CS IA to IIIB non-bulky Hodgkin's disease (NB-HD) with 3 cycles of chemotherapy (CT) (ABVD vs EBVM) followed by high dose irradiation (RT). Results of the GOELAMS H90-NM multicentre randomized trial. Blood 1999;94(10 suppl. 1):abstract 386a.

\section{Dionet 1988 published data only}

Dionet C, Oberlin O, Habrand JL, Vilcoq J, Madelain M, Dutou $\mathrm{L}$, et al.Initial chemotherapy and low-dose radiation in limited fields in childhood Hodgkin's disease: results of a joint cooperative study by the French Society of Pediatric Oncology (SFOP) and Hôpital Saint-Louis, Paris. International Journal of Radiation Oncology, Biology, Physics 1988;15(2):341-6.

Hirsch 1996 \{published data only\}

Hirsch A. The effect of ABVD chemotherapy with and without mediastinal irradiation on pulmonary function and symptoms in early-stage Hodgkin's disease. International Journal of Radiation Oncology, Biology, Physics 1994;30(suppl. 1):168.

Hirsch A, Vander EN, Straus DJ, Gomez EG, Leung D, Portlock CS, et al.Effect of ABVD chemotherapy with and without mantle or mediastinal irradiation on pulmonary function and symptoms in early-stage Hodgkin's disease. Journal of Clinical Oncology 1996;14 (4):1297-305.

Horning 1996 \{published data only\}

Horning SJ, Bennett JM, Bartlett NL, Williams J, Neuberg D, Cassileth PA. 12 weeks of chemotherapy (STANFORD V) and involved field radiotherapy (RT) are highly effective for bulky and advanced stage Hodgkin's disease (HD): a limited institution ECOG pilot study. Blood 1996;88(10 Suppl (Pt 1)):673a.

\section{Horning 2007 \{published data only\}}

Horning SJ, Hoppe RT, Advani RH, Breslin S, McCormick E, Allen J, et al.A prospective trial of involved field radiation (IFRT) + chemotherapy vs. extended field radiation (EFRT) for favorable Hodgkin's disease (HD): Long-term follow-up and implications for current combined modality. Haematologica 2007;92(suppl. 5):53.

\section{Kim 2003 \{published data only\}}

Kim HK, Silver B, Li S, Neuberg D, Mauch P. Hodgkin's disease in elderly patients ( $>$ or $=60$ ): clinical outcome and treatment strategies. International Journal of Radiation Oncology, Biology, Physics 2003;56(2):556-60.

\section{Koerholz 2004 \{published data only\}}

Korholz D, Claviez A, Hasenclever D, Kluge R, Hirsch W, Kamprad F, et al.The concept of the GPOH-HD 2003 therapy study for pediatric Hodgkin's disease: evolution in the tradition of the DAL/GPOH studies. Klinische Pädiatrie 2004;216(3):150-6.

\section{Kung 1993 \{published data only\}}

Kung FH, Behm FG, Cantor A, Falletta J, Ferree CR, Leventhal $\mathrm{BG}$, et al.Abbreviated chemotherapy vs chemoradiotherapy in early stage Hodgkin's disease of childhood. Proceedings of the American Society of Clinical Oncology. 1993; Vol. 12:414.

\section{Kung 2006 \{published data only\}}

Kung FH. POG 8625: A randomized trial comparing chemotherapy with chemoradiotherapy for children and adolescents with stages I, IIA, IIIA Hodgkin disease: A report from the children's oncology group. Journal of Pediatric Hematology/ Oncology 2006;28(6):362-8.

\section{Laskar 2004 \{published data only\}}

Laskar S, Gupta T, Vimal S, Muckaden MA, Saikia TK, Pai SK, et al.Consolidation radiation after complete remission in Hodgkin's disease following six cycles of doxorubicin, bleomycin, vinblastine, and dacarbazine chemotherapy: is there a need?. Journal of Clinical Oncology 2004;22(1):62-8.

\section{Longo 1992 \{published data only\}}

Longo DL, DeVita VT. The use of combination chemotherapy in the treatment of early stage Hodgkin's disease. In: De Vita VT, Helman S, Rosenberg SA editor(s). Important Advances in Oncology. Philadelphia: Lippincott Williams \& Wilkins, 1992:155-65.

Meyer 2005 \{published data only\}

Meyer RM, Gospodarowicz MK, Connors JM, Pearcey RG, Bezjak A, Wells WA, et al.Randomized comparison of ABVD chemotherapy with a strategy that includes radiation therapy in patients with limited-stage Hodgkin's lymphoma: National Cancer Institute of Canada Clinical Trials Group and the Eastern Cooperative Oncology Group. Journal of Clinical Oncology 2005; 23(21):4634-42.

Portlock CS. Clinical trials report. Comparison of ABVD chemotherapy and a regimen including radiation therapy in patients with limited-stage non-Hodgkin's lymphoma. Current Oncology Reports 2006;8(5):354-7.

\section{Nachman 2002 \{published data only\}}

Nachman JB, Sposto R, Herzog P, Gilchrist GS, Wolden SL, Thomson J, et al.Randomized comparison of low-dose involved- 
field radiotherapy and no radiotherapy for children with Hodgkin's disease who achieve a complete response to chemotherapy. Journal of Clinical Oncology 2002;20(18):3765-71.

Noordijk 2006 \{published data only\}

Noordijk EM, Carde P, Dupouy N, Hagenbeek A, Krol AD, KluinNelemans JC, et al.Combined-modality therapy for clinical stage I or II Hodgkin's lymphoma: long-term results of the European Organisation for Research and Treatment of Cancer H7 randomized controlled trials. Journal of Clinical Oncology 2006;24 (19):3128-35.

O'Dwyer 1984 \{published data only\}

O'Dwyer PJ, Stewart MB, Wiernik PH. MOPP vs radiotherapy/ MOPP for early-stage Hodgkin's disease (HD) - a six year followup. 2nd International Conference on Malignant Lymphoma, Lugano, Switzerland. June 13, 1984; Vol. 16:46.

O'Dwyer 1985 \{published data only\} O’Dwyer PJ, Wiernik PH, Stewart MB, Slawson RG. Treatment of early stage Hodgkin's disease: a randomized trial of radiotherapy plus chemotherapy versus chemotherapy alone. In: Cavilli F, Bonadonna G, Rozencweig M editor(s). Malignant Lymphomas and Hodgkin's Disease: Experimental and Therapeutic Advances. Boston: Maritinus Nijhoff, 1985:329-36.

Pavlovsky 1997 \{published data only\}

Pavlovsky S, Schvartzman E, Lastiri F, Magnasco H, Corrado C, Raslawski E, et al.Randomized trial of CVPP for three versus six cycles in favorable-prognosis and CVPP versus AOPE plus radiotherapy in intermediate-prognosis untreated Hodgkin's disease. Journal of Clinical Oncology 1997;15(7):2652-8.

Picardi 2007 \{published data only\}

Picardi M, De Renzo A, Pane F, Nicolai E, Pacelli R, Salvatore M, et al.Randomized comparison of consolidation radiation versus observation in bulky Hodgkin's lymphoma with post-chemotherapy negative positron emission tomography scans. Leukemia o Lymphoma 2007;48(9):1721-7.

Radford 2002 \{published data only\} Radford JA, Cowan RA, Ryder WD, Johnson RJ, Bannerjee SS, Deakin DP, et al.Four weeks of VAPEC-B chemotherapy before involved field radiotherapy minimises the relapse rate in early stage low-risk Hodgkin's disease and is not associated with an excess of second malignancy. Annals of Oncology 2002;13(suppl. 2):25.

Rüffer 1996 \{published data only\}

Rüffer U, Brosteanu O, Sieber M, Koch T, Löffler M, Pfreundschuh M. Reduction of radiotherapy in early stage Hodgkin's disease: results of a randomized trial in patients ps I/II. Annals of Oncology 1996;7(suppl. 3):49.

Rüffer 1998 \{published data only\}

Rüffer U, Sieber M, Pfistner B, Tesch H, Engert A, Bredenfeld H, et al.Reduction of radiotherapy volume in intermediate Hodgkin's disease: Interim analysis of a randomized trial in patients CS I/II of the GHSG. Blood 1998;92(10 suppl 1 (Pt 1)):abstract 626a.

Rüffer 1999 \{published data only\}

Rüffer JU, Sieber M, Pfistner B, Tesch H, Engert A, Bredenfeld H, et al.For intermediate stage Hodgkin's disease extended field radiation after effective chemotherapy is obsolete: interim analysis of HD9 trial (GHSG). Blood 1999;94(10 suppl. 1):528a.
Specht 1992 \{published data only\}

Specht L, Carde P, Mauch P, Magrini SM, Santarelli MT.

Radiotherapy versus combined modality in early stages. Annals of Oncology 1992;3(suppl. 4):77-81.

Straus 1989 \{published data only\}

Straus DJ, Myers J, Lee BJ, Koziner B, Nisce LZ, Redman J. Limited chemotherapy and radiation therapy (RT) for early clinical stage (CS) Hodgkin's disease (HD). High complete remission (CR) percentage, disease free survival (DFS) and low toxicity. Blood 1989;74(7 suppl. 1):239a.

Thistlethwaite 2007 \{published data only\}

Thistlethwaite F, Qian W, Williams MV, Hancock BW, Hoskin P, Sun-Mynt H, et al.Selection of patients for minimal initial chemotherapy (MIC); the impact of Hasenclever score on outcome in patients receiving MIC and involved field radiotherapy for clinical stage IA/IIA supra-diaphragmatic Hodgkin lymphoma in the UK NCRI LY07 trial. Haematologica 2007;92(suppl. 5):52.

\section{References to ongoing studies}

EORTC-GELA HD 10 \{published data only\} EORTC-GELA. The H10 EORTC/GELA Randomized Intergroup Trial on Early FDG-PET Scan Guided Treatment Adaptation Versus Standard Combined Modality Treatment in Patients With Supradiaphragmatic Stage I/II Hodgkin's Lymphoma. www.clinicaltrials.gov NCT00433433.

\section{Friedman \{published data only\}}

Friedman D. Phase III Randomized Study of Response-Based Chemotherapy With Doxorubicin, Bleomycin, Vincristine, Etoposide, Prednisone, and Cyclophosphamide With or Without Dexamethasone, Etoposide, Cytarabine, Cisplatin, and/or Radiotherapy in Children With Newly Diagnosed IntermediateRisk Hodgkin's Lymphoma. National Institutes of Health, ClinicalTrials Gov [http://www clinicaltrials.gov] 2003.

\section{GHSG HD 16 \{published data only\}}

GHSG. HD16 for Early Stages - Treatment Optimization Trial in the First-Line Treatment of Early Stage Hodgkin Lymphoma; Treatment Stratification by Means of FDG-PET. www.clinicaltrials.gov NCT00736320.

\section{Additional references}

\section{Adams 2004}

Adams MJ, Lipsitz SR, Colan SD, Tarbell NJ, Treves ST, Diller L, et al.Cardiovascular status in long-term survivors of Hodgkin's disease treated with chest radiotherapy. Journal of Clinical Oncology 2004;22(15):3139-48

Aleman 2003

Aleman BM, van den Belt-Dusebout AW, Klokman WJ, Van't Veer $\mathrm{MB}$, Bartelink $\mathrm{H}$, van Leeuwen FE. Long-term cause-specific mortality of patients treated for Hodgkin's disease. Journal of Clinical Oncology 2003;21(18):3431-9.

Altman 1999

Altman DG, Andersen PK. Calculating the number needed to treat for trials where the outcome is time to an event. BMJ 1999;319: 1492-5. 


\section{Bhatia 2003}

Bhatia S, Yasui Y, Robison LL, Birch JM, Bogue MK, Diller L, et al.High risk of subsequent neoplasms continues with extended follow-up of childhood Hodgkin's disease: report from the Late Effects Study Group. Journal of Clinical Oncology 2003;21(23): 4386-94.

\section{Canellos 2005}

Canellos GP. Chemotherapy alone for early Hodgkin's lymphoma: an emerging option. Journal of Clinical Oncology 2005;23(21): 4574-6.

Carbone 1971

Carbone PP, Kaplan HS, Musshoff K, Smithers DW, Tubiana M. Report of the Committee on Hodgkin's Disease Staging Classification. Cancer Research 1971;31(11):1860-61.

\section{Chaber 2006}

Chaber BA, Amrein PC, Druker BJ, Michaelson MD, Mitsiades CS, Goss PF, et al.Antineoplastic agents. In: Brunton LL, Lazo JS, Parker KL editor(s). Goodman and Gillman's. The pharmacological basis of therapeutics. 11. McGraw-Hill, Medical Publishing Division, 2006:1380.

\section{Cheson 2007}

Cheson BD, Pfistner B, Juweid ME, Gscoyne RD, Specht L, Horning SJ, et al.Revised response criteria for malignant lymphoma. Journal of Clinical Oncology 2007;25(5):579-86.

\section{Connors 2001}

Connors JM, Noordijk EM, Horning SJ. Hodgkin's lymphoma: basing the treatment on the evidence. Hematology: American Society of Haematology Education Book. 2001:178-93.

\section{Connors 2005}

Connors JM. State-of-the-art therapeutics: Hodgkin's lymphoma. Journal of Clinical Oncology 2005;23(26):6400-8.

\section{Deeks 2008}

Deeks JJ, Higgins JPT, Altman DG. Chapter 9: Analysing data and undertaking meta-analyses. In: Higgins JPT, Green $S$ editor(s). Cochrane Handbook for Systematic Reviews of Interventions. Chichester: John Wiley \& Sons, 2008:243-96.

\section{DeVita 1997}

DeVita VT Jr, Mauch PM, Harris NL. Hodgkin's disease. In: DeVita VT Jr, Hellmann S, Rosenberg SA editor(s). Cancer principles and practice of oncology. 5th Edition. Philadelphia: Lippincott-Raven, 1997:2242-83.

\section{Dickersin 1993}

Dickersin K, Min YI. NIH clinical trials and publication bias. Online Journal of Current Clinical Trials 1993;50:4967.

\section{Diehl 2005}

Diehl V, Re D, Josting A. Hodgkin's disease: clinical manifestations, staging, and therapy. In: Hoffman R, Benz EJ, Shattil SJ, Furie B, Cohen HJ editor(s). Hematology: basic principles and practice. 4th Edition. Churchill Livingstone, 2005:1347-78.

\section{Dores 2002}

Dores GM, Metayer C, Curtis RE, Lynch CF, Clarke EA, Glimelius $\mathrm{B}$, et al.Second malignant neoplasms among long-term survivors of Hodgkin's disease: a population-based evaluation over 25 years. Journal of Clinical Oncology 2002;20(16):3484-94.

\section{Egger 1997}

Egger M, Zellweger-Zahner T, Schneider M, Junker C, Lengeler C, Antes G. Language bias in randomised controlled trials published in English and German. Lancet 1997;350(9074):326-9.

Egger 1997a

Egger M, Smith GD, Phillips AN. Meta-analysis: principles and procedures. British Medical Journal 1997;315(7121):1533-7.

\section{Engert 2003}

Engert A, Schiller P, Josting A, Herrmann R, Koch P, Sieber M, et al.Involved-Field Radiotherapy Is Equally Effective and Less Toxic Compared With Extended-Field Radiotherapy After Four Cycles of Chemotherapy in Patients With Early-Stage Unfavorable Hodgkins Lymphoma: Results of the HD8 Trial of the German Hodgkin's Lymphoma Study Group. Journal of Clinical Oncology 2003;21 (19):3601-8.

\section{Engert 2007}

Engert A, Franklin J, Eich HT, Brillant C, Sehlen S, Cartoni C, et al.Two cycles of doxorubicin, bleomycin, vinblastine, and dacarbazine plus extended-field radiotherapy is superior to radiotherapy alone in early favorable Hodgkin's lymphoma: final results of the GHSG HD7 trial. Journal of Clinical Oncology 2007; 25(23):3495-502.

Franklin 2005

Franklin JG, Paus MD, Pluetschow A, Specht L. Chemotherapy, radiotherapy and combined modality for Hodgkin's disease, with emphasis on second cancer risk. Cochrane Database of Systematic Reviews 2005, Issue 4. [DOI: 10.1002/14651858.CD003187]

\section{Glaser 1996}

Glaser SL, Jarrett RF. The epidemiology of Hodgkin's disease. Baillière's Clinical Haematology 1996;9(3):401-16.

\section{Green 2000}

Green DM, Hyland A, Barcos MP, Reynolds JA, Lee RJ, Hall BC, et al.Second malignant neoplasms after treatment for Hodgkin's disease in childhood or adolescence. Journal of Clinical Oncology 2000;18(7):1492-9.

\section{Hahn 2005}

Hahn SM, Glastein E. Principles of radiation therapy. In: Kasper DL, Fauci AS, Longo DL, Braunwald E, Hauser SL, Jameson JL editor(s). Harrison's principles of internal medicine. 16. New York: McGraw-Hill, Medical Publishing Division, 2005:482-9.

\section{Higgins 2002}

Higgins JP, Thompson SG. Quantifying heterogeneity in a metaanalysis. Statistics in Medicine 2002;21(11):1539-58.

\section{Higgins 2003}

Higgins JP, Thompson SG, Deeks JJ, Altman DG. Measuring inconsistency in meta-analyses. British Medical Journal 2003;327 (7414):557-60.

\section{Higgins 2008}

Higgins JPT, Altman DG. Chapter 8: Assessing risk of bias in included studies. In: Higgins JPT, Green S editor(s). Cochrane Handbook for Systematic Reviews of Interventions. Chichester: John Wiley \& Sons, 2008:187-241.

\section{Juni 2002}

Juni P, Holenstein F, Sterne J, Bartlett C, Egger M. Direction and impact of language bias in meta-analyses of controlled trials: 
empirical study. International Journal of Epidemiology 2002;31(1): $115-23$.

\section{Klimm 2005}

Klimm B, Engert A, Diehl V. First-line treatment of Hodgkin's lymphoma. Current Hematology Reports 2005;4(1):15-22.

\section{Lister 1989}

Lister TA, Crowther D, Sutcliffe SB, Glatstein E, Canellos GP, Young RC, et al.Report of a committee convened to discuss the evaluation and staging of patients with Hodgkin's disease: Cotswolds meeting. Journal of Clinical Oncology 1989;7(11): $1630-6$.

\section{Lukes 1966}

Lukes RJ, Butler JJ. The pathology and nomenclature of Hodgkin's disease. Cancer Research (1966);26:1063-83.

\section{Mauch 1999}

Mauch PM, Armitage JO, Diehl V, Hoppe RT, Weiss LM. Hodgkin's disease. 1st Edition. Philadelphia: Lippicott Williams \& Wilkins, 1999.

\section{Meyer 2005a}

Meyer RM. Is there convincing evidence for the use of chemotherapy alone in patients with limited stage Hodgkin's lymphoma?. European Journal of Haematology 2005;66(suppl.): $115-20$.

\section{Moher 1999}

Moher D, Cook DJ, Eastwood S, Olkin I, Rennie D, Stroup DF. Improving the quality of reports of meta-analyses of randomised controlled trials: the QUOROM statement. Quality of Reporting of Meta-analyses. Lancet 1999;354(9193):1896-900.

\section{Mueller 1999}

Mueller NE, Gruffermann S. The epidemiology of Hodgkin's disease. In: Mauch PM, Armitage JO, Diehl V, Hoppe RT, Weiss LM editor(s). Hodgkin's disease. 1st Edition. Philadelphia: Lippicott Williams \& Wilkins, 1999:61-78.

Ng 2002a

Ng AK, Bernardo MP, Weller E, Backstrand KH, Silver B, Marcus $\mathrm{KC}$, et al.Long-term survival and competing causes of death in patients with early-stage Hodgkin's disease treated at age 50 or younger. Journal of Clinical Oncology 2002;20(8):2101-8.

\section{$\mathrm{Ng} 2002 b$}

$\mathrm{Ng}$ AK, Bernardo MV, Weller E, Backstrand K, Silver B, Marcus $\mathrm{KC}$, et al.Second malignancy after Hodgkin disease treated with radiation therapy with or without chemotherapy: long-term risks and risk factors. Blood 2002;100(6):1989-96.

\section{Parmar 1998}

Parmar MK, Torri V, Stewart L. Extracting summary statistics to perform meta-analyses of the published literature for survival endpoints. Statistics in Medicine 1998;17(24):2815-34.

\section{Sausville 2005}

Sausville EA, Longo DL. Principles of cancer treatment: surgery, chemotherapy and biologic therapy. In: Kasper DL, Fauci AS, Longo DL, Braunwald E, Hauser SL, Jameson JL editor(s). Harrison's principles of internal medicine. 16. New York: McGrawHill, Medical Publishing Division, 2005:464-83.

\section{Singal 1998}

Singal PK, Iliskovic N. Doxorubicin-induced cardiomyopathy. New England Journal of Medicine 1998;339(13):900-5.

\section{Specht 1998}

Specht L, Gray RG, Clarke MJ, and Peto R for the International Hodgkin's Disease Collaborative Group. Influence of more extensive radiotherapy and adjuvant chemotherapy on long-term outcome of early-stage Hodgkin's disease: A meta-analysis of 23 randomized trials involving 3,888 patients. Journal of Clinical Oncology 198;16:830-43.

Swerdlow 2000

Swerdlow AJ, Barber JA, Hudson GV, Cunningham D, Gupta RK, Hancock BW, et al.Risk of second malignancy after Hodgkin's disease in a collaborative British cohort: the relation to age at treatment. Journal of Clinical Oncology 2000;18(3):498-509.

\section{Swerdlow 2003}

Swerdlow AJ. Epidemiology of Hodgkin's disease and nonHodgkin's lymphoma. European Journal of Nuclear Medicine \& Molecular Imaging 2003;30 Suppl 1:S3-12.

\section{Thomas 2002}

Thomas RK, Re D, Zander T, Wolf J, Diehl V. Epidemiology and etiology of Hodgkin's lymphoma. Annals of Oncology 2002;13 Suppl 4:147-52.

Tierney 2007

Tierney JF, Stewart LA, Ghersi D, Burdett S, Sydes MR. Practical methods for incorporating summary time-to-event data into metaanalysis. Trials 2007;8(16):1745-62.

\section{van Leeuwen 2000}

van Leeuwen FE, Klokman WJ, Veer MB, Hagenbeek A, Krol AD, Vetter UA, et al.Long-term risk of second malignancy in survivors of Hodgkin's disease treated during adolescence or young adulthood. Journal of Clinical Oncology 2000;18(3):487-97.

* Indicates the major publication for the study 
CHARACTERISTICS OF STUDIES

Characteristics of included studies [ordered by study ID]

\section{CALGB 7751}

Methods

- Randomised controlled trial with two arms: CT-alone arm and CMT arm.

- Conducted by the Cancer and Leukemia Group B (CALGB), USA.

- Recruitment period in 1970 s, exact period unclear.

- 55 patients allocated; exact number per arm not reported.

- 37 patients evaluated: 18 patients in CT-alone arm and 19 patients in CMT arm.

- Baseline patient's characteristics described.

- Median follow-up time: 22 months.

- Not ITT analysis; more than $10 \%$ of the enrolled patients not evaluated.

Participants

- Inclusion criteria: patients with histologically documented, previously untreated, poor prognosis pathological stage I and I; poor prognosis was defined as symptom class $\mathrm{B}$, mixed cellularity or lymphocyte depleted histology, a large mediastinal mass, or age $>40$ years

- Exclusion criteria: not reported.

- PS I, II: CT-alone: 1, 17; CMT: 6, 13.

- Prognostic features: not reported.

- Mean age: CT-alone: 24 years; CMT: 30 years;

- Gender male, female: CT-alone: 6, 12; CMT: 14; 5 .

- Baseline patient's characteristics: more male patients in CMT arm; more patients with mediastinal mass in CT-alone arm.

- Histopathologic diagnosis: according to Rye modification of Lukes and Butler classification.

Interventions

- CT-alone: 6 cycles of CVPP (cyclophosphamide $\left(75 \mathrm{mg} / \mathrm{m}^{2}\right.$ orally, day 1$)$, vinblastine $\left(4 \mathrm{mg} / \mathrm{m}^{2}\right.$ intravenous, days 1 and 8$)$, procarbazine $\left(100 \mathrm{mg} / \mathrm{m}^{2}\right.$ orally, days 1-14), prednisone ( $40 \mathrm{mg} / \mathrm{m}^{2}$ orally, days $\left.1-14\right)$ ); repeated every 14 days.

- CMT: same CT with involved-field radiotherapy; dose of radiotherapy (RT) not reported; RT delivered before CT.

- No additional treatment.

Outcomes

- Overall survival reported.

- Complete response reported.

- Partial response not reported.

- Progression-free survival not reported.

Notes

- Response documented after two cycles of CT.

- Source of funding not reported.

Risk of bias

\begin{tabular}{lll}
\hline Item & Authors' judgement & Description \\
\hline Adequate sequence generation? & Unclear & "randomly allocated” \\
\hline Allocation concealment? & Unclear & Not reported. \\
\hline C & &
\end{tabular}


CALGB 7751 (Continued)

\begin{tabular}{l|l|l}
\hline $\begin{array}{l}\text { Blinding? } \\
\text { Overall survival }\end{array}$ & Yes & $\begin{array}{l}\text { Patients not blinded. No information } \\
\text { about blinding of the assessor. This is } \\
\text { judged not to be a source of bias for overall } \\
\text { survival. }\end{array}$ \\
\hline $\begin{array}{l}\text { Blinding? } \\
\text { Other outcomes }\end{array}$ & Unclear & $\begin{array}{l}\text { Patients not blinded. No information } \\
\text { about blinding of the assessor. }\end{array}$ \\
\hline $\begin{array}{l}\text { Incomplete outcome data addressed? } \\
\text { All outcomes }\end{array}$ & No & $\begin{array}{l}22 \text { months OS and response outcome: 18/ } \\
55 \text { missing from the outcome analysis; no } \\
\text { information per study arm. This trial was } \\
\text { considered not to have performed an ITT } \\
\text { analysis in the subgroup analysis. }\end{array}$ \\
\hline Free of selective reporting? & Unclear & $\begin{array}{l}\text { Dates of relapse and deaths are given. Dates } \\
\text { of progression not given nor information } \\
\text { about censoring. No time to event out- } \\
\text { comes calculated. }\end{array}$ \\
\hline Free of other bias? & Unclear & \begin{tabular}{l} 
Very little information in the report. \\
\hline
\end{tabular}
\end{tabular}

\section{EORTC-GELA H9-F}

Methods

- A randomised controlled trial with three arms: comparison of three radiation dosis; 36 Gy involved-field radiotherapy, 20 Gy involved-field radiotherapy and no radiotherapy in patients that achieved complete response (CR) after six cycles of EBVP.

- Conducted by EORTC (European Organization for Research and Treatment of Cancer) and GELA (Groupe d'Etude des Lymphomes de l'Adulte); 111 institutions from 10 European countries involved.

- Recruitment period from September 1998 to May 2004.

- 784 patients enrolled.

- 13 patients not evaluable before randomisation (6 refusals, 3 protocol violations, 4 unspecified).

- 578 patients randomised to three radiation doses.

- 578 patients evaluated.

- Baseline patient's characteristics not reported (abstract publication).

- Median follow-up 51 months (range 14 to 81 ).

- ITT analysis.

Participants

- Inclusion criteria: adult patients with supradiaphragmatic CS I-II Hodgkin lymphoma and favourable features (age $<50$, CS I-II, symptoms class A + ESR $<50$ or symptoms class $\mathrm{B}+\mathrm{ESR}<30$ and MT ratio $<0.35$ ).

- Exclusion criteria: not reported.

- Age: 31 (15 to 49)

- Gender: $55 \%$ male; $45 \%$ female

- CS: patients with CS I-II without bulky disease.

- Prognostic features: all included patients with favourable risk factors.

- Histopathologic diagnosis: not reported. 
EORTC-GELA H9-F (Continued)

\begin{tabular}{|c|c|}
\hline Interventions & $\begin{array}{l}\text { - CT-alone: } 6 \text { cycles of EBVP (epirubicin }\left(70 \mathrm{mg} / \mathrm{m}^{2} \text { intravenous, day } 1\right) \text {, } \\
\text { bleomycin }\left(10 \mathrm{mg} / \mathrm{m}^{2} \text { intravenous/intramuscular, day } 1\right) \text {, vinblastine }\left(6 \mathrm{mg} / \mathrm{m}^{2}\right. \\
\left.\text { intravenous, day } 1) \text {, prednisone ( } 40 \mathrm{mg} / \mathrm{m}^{2} \text { orally, day } 1-5\right) \text { ); repeated after every } 21 \\
\text { days. } \\
\text { - CMT: same CT before randomisation with } 36 \text { Gy involved-field radiotherapy or } \\
20 \text { Gy involved-field radiotherapy. } \\
\text { - No additional treatment. }\end{array}$ \\
\hline Outcomes & $\begin{array}{l}\text { - Overall survival reported; observation time } 4 \text { years. } \\
\text { - Response not reported. } \\
\text { - Progression-free survival not reported. } \\
\text { - Disease-free survival reported (Table 3). }\end{array}$ \\
\hline Notes & $\begin{array}{l}\text { - Inclusion of patients in no radiotherapy arm was stopped in May } 2002 \text { because } \\
\text { stopping rules were met that is }>20 \% \text { events occurred. } \\
\text { - Hazard ratio estimate is based on the full group receiving additional radiotherapy } \\
\text { and not only those patients up to the time the no radiotherapy arm was stopped. } \\
\text { - Source of funding not reported. }\end{array}$ \\
\hline
\end{tabular}

Risk of bias

\begin{tabular}{|c|c|c|}
\hline Item & Authors' judgement & Description \\
\hline Adequate sequence generation? & Yes & $\begin{array}{l}\text { Patients were randomly allocated. No fur- } \\
\text { ther information available. However it was } \\
\text { a large multicenter trial with presumably } \\
\text { central allocation (older trials of the same } \\
\text { group). We therefore assume that it was } \\
\text { probably ok. }\end{array}$ \\
\hline
\end{tabular}

Allocation concealment?

Blinding?

Overall survival

\section{Blinding?}

Other outcomes
Yes

Yes

Unclear

No information available from the publications. However, it is a large multicenter trial with 120 centres. Older trials from this group were centrally allocated. We therefore assume that it was probably ok.

Patients not blinded (not expected due to the treatment with radiotherapy). No information about blinding of the assessor. This is judged not to be a source of bias for overall survival.

Patients and physicians not blinded (not expected due to the treatment with radiotherapy). No information about blinding of the assessor. 
EORTC-GELA H9-F (Continued)

\begin{tabular}{|l|l|l}
\hline $\begin{array}{l}\text { Incomplete outcome data addressed? } \\
\text { All outcomes }\end{array}$ & Yes & $\begin{array}{l}\text { No withdrawals and protocol violations af- } \\
\text { ter randomisation reported. Analysis was } \\
\text { performed on ITT basis and all randomised } \\
\text { patients were included in the analysis. }\end{array}$ \\
\hline Free of selective reporting? & Yes & $\begin{array}{l}\text { Rationale for the use of disease-free survival } \\
\text { not described. However all patients are in } \\
\text { CR at the time of randomisation. Disease- } \\
\text { free survival should therefore be equivalent } \\
\text { to progression free survival. Other progres- } \\
\text { sion outcomes that are more prone to bias } \\
\text { are not used and not reported. Despite no } \\
\text { protocol being available, we judge that the } \\
\text { trial is free of selective outcome reporting. }\end{array}$ \\
\hline Free of other bias? & No & $\begin{array}{l}\text { Trial was ended early due to predefined } \\
\text { stopping rule. This is known to increase the } \\
\text { effect estimate of trials. In addition the data } \\
\text { are preliminary. }\end{array}$ \\
\hline
\end{tabular}

\section{GATLA 9-H-77}

Methods
- A randomised controlled trial with two arms: CT-alone arm and CMT arm.

- Conducted by Group Argentino de Tratamientode la Leucemia Aguda (GATLA).

- Recruitment period from September 1977 to October 1986.

- 293 patients randomised: CT-alone:148; CMT: 145.

- 277 patents evaluated: 142 in CT-alone arm and 135 in CMT arm.

- 16 patients not evaluated; 4 drop outs and 2 protocol violations in CT-alone arm; 9 drop outs and one protocol violation in CMT arm.

- Baseline patient's characteristics described.

- Median time on study was 43 months for CT-alone arm and 51 months for CMT arm.

- Not ITT analysis; less than $10 \%$ of enrolled patients not evaluated.

Inclusion criteria: patients with pathologically proven Hodgkin lymphoma, according to Lukes and Butler's criteria.

- Exclusion criteria: patients with CS III.

- CS I, II: CT-alone: 47, 95; CMT: 46, 89; overall CS I 93, CS II 184.

- Prognostic features: CT-alone: 82 patients with early favourable stage; 60 patients with early unfavourable stage; CMT: 91 patients with early favourable stage; 44 patients with early unfavourable stage.

- Age: patients of all ages; median age not reported; 124 patients (45\%) were children $<16$ years.

- Gender: CT-alone: 87 males, 55 females; CMT: 88 males, 47 females.

- Similar baseline patient's characteristics in comparison arms.

- Histopathologic diagnosis: according to the Rye modification of the Lukes and Butler. 
GATLA 9-H-77 (Continued)

\begin{tabular}{ll}
\hline Interventions & $\begin{array}{l}\text { - CT-alone: } 6 \text { monthly cycles of CVPP (cyclophosphamide }\left(600 \mathrm{mg} / \mathrm{m}^{2}\right. \\
\text { intravenous, day 1), vinblastine }\left(6 \mathrm{mg} / \mathrm{m}^{2} \text { intravenous, day 1), procarbazine }(100 \mathrm{mg} /\right. \\
\left.\mathrm{m}^{2} \text { orally, days } 1-14\right), \text { prednisone }\left(40 \mathrm{mg} / \mathrm{m}^{2} \text { orally, days 1-14)). }\right. \\
\text { - CMT: same CT with IF-RT between third and fourth cycle of CT (sandwich } \\
\text { technique); dose of RT was } 3000 \mathrm{rad} . \\
\text { - No additional treatment. }\end{array}$ \\
\hline Outcomes & $\begin{array}{l}\text { - Overall survival reported also for prognostic groups; observation time } 7 \text { years. } \\
\text { - Complete response reported. } \\
\text { - Partial response reported. } \\
\text { - Progression-free survival not reported. } \\
\text { - "Disease-free survival" reported (see Table 3) }\end{array}$ \\
\hline Notes & - Limited overall survival data for prognostic groups; estimation of HR not possible. \\
\hline
\end{tabular}

\section{Risk of bias}

\begin{tabular}{|c|c|c|}
\hline Item & Authors' judgement & Description \\
\hline Adequate sequence generation? & Unclear & $\begin{array}{l}\text { "[...] patients }[\ldots] \text { were randomised }[\ldots] " \\
\text { No further information available. }\end{array}$ \\
\hline Allocation concealment? & Unclear & No information available. \\
\hline $\begin{array}{l}\text { Blinding? } \\
\text { Overall survival }\end{array}$ & Yes & $\begin{array}{l}\text { Patients not blinded. No information } \\
\text { about blinding of the assessor. This is } \\
\text { judged not to be a source of bias for overall } \\
\text { survival. }\end{array}$ \\
\hline $\begin{array}{l}\text { Blinding? } \\
\text { Other outcomes }\end{array}$ & Unclear & $\begin{array}{l}\text { Patients not blinded. No information } \\
\text { about blinding of the assessor. }\end{array}$ \\
\hline $\begin{array}{l}\text { Incomplete outcome data addressed? } \\
\text { All outcomes }\end{array}$ & Yes & $\begin{array}{l}5 \text { years OS and response rate: } 6 / 142 \text { miss- } \\
\text { ing from CT-alone arm and } 10 / 135 \text { from } \\
\text { CMT arm; } 13 \text { lost to follow-up before } \\
\text { completing } 6 \text { cycles and } 3 \text { protocol viola- } \\
\text { tions (not described in detail); } \\
\text { Therefore for subgroup analysis this trial } \\
\text { was considered not to have performed an } \\
\text { ITT analysis. } \\
\text { Despite the authors not addressing the } \\
\text { missing outcomes in analyses, we judge that } \\
\text { these few missing data have very little in- } \\
\text { fluence on the overall results. }\end{array}$ \\
\hline Free of selective reporting? & Unclear & $\begin{array}{l}\text { For progression outcome, disease-free sur- } \\
\text { vival was chosen. No other progression out- } \\
\text { comes are reported but it is unclear why dis- }\end{array}$ \\
\hline
\end{tabular}


GATLA 9-H-77 (Continued)

\begin{tabular}{l|l|l} 
& & $\begin{array}{l}\text { ease-free survival was chosen and not pro- } \\
\text { gression free survival. No protocol is avail- } \\
\text { able. }\end{array}$ \\
\hline Free of other bias? & Yes & $\begin{array}{l}\text { From the report, the trial seems free of other } \\
\text { bias. }\end{array}$ \\
\hline
\end{tabular}

\section{Mexico B2H031}

Methods

- Randomised controlled trial with three arms: CT-alone arm, CMT arm and RTalone arm.

- Conducted at Oncology Hospital, National Medical Center, Mexico.

- Recruitment period from 1983 to 1988.

- 327 patients enrolled.

- 307 patients evaluated; 99 patients in CT-alone arm; 102 patients in CMT arm and 106 patients in RT-alone arm.

- 20 patients not evaluated due to advanced stage and infradiaphragmatic involvement.

- Baseline patient characteristics described.

- Median follow-up time 11.4 years (range 6.3 - 16.5 years).

- Not ITT analysis; less than $10 \%$ of enrolled patients not evaluated.

Participants

- Inclusion criteria: both male and female adult patients with clinical stage I and II (CS I and II), supradiaphragmatic disease and the presence of bulky disease.

- Exclusion criteria: patients with advanced stages and infradiaphragmatic involvement.

- CS I, II: CT-alone: 21, 78; CMT: 22, 80; overall CS I 34\%, CS II 66\%.

- Prognostic features not reported.

- Mean age (range): CT-alone: 39 (20 to 70) years; CMT: 42 (18 to 71) years.

- Gender: CT-alone: 40 males, 59 females; CMT: 51 males, 51 females.

- Similar baseline patient's characteristics in comparison arms.

- Histopathologic diagnosis: according to Rye modification of Lukes and Butler classification.

Interventions

- CT-alone: 6 monthly cycles of ABVD (adriamycin, bleomycin, vinblastine, dacarbazine); dose not reported.

- CMT: same CT with mantle-field radiotherapy (MF-RT) between third and fourth cycles of CT (sandwich technique); dose of RT: 3500-3800 cGy in fraction of 200-250 cGy four to five times a week for four to six weeks.

- RT-alone: EF-RT with a dose of 3500-3800 cGy in fraction of 200-250 cGy four to five times a week over a period of four weeks; 106 patients from this arm not included in the review.

- No additional treatment.

Outcomes

- Overall survival reported; observation time 12 years.

- Complete response reported.

- Partial response reported.

- Progression-free survival not reported.

- Contradictory definitions of disease-free survival (see Table 3) 
Mexico B2H031 (Continued)

\begin{tabular}{|c|c|c|}
\hline Notes & \multicolumn{2}{|c|}{ - Source of funding not reported. } \\
\hline \multicolumn{3}{|l|}{ Risk of bias } \\
\hline Item & Authors' judgement & Description \\
\hline Adequate sequence generation? & Unclear & $\begin{array}{l}\text { "a prospective randomised trial" No further } \\
\text { information available. }\end{array}$ \\
\hline Allocation concealment? & Unclear & No information available. \\
\hline $\begin{array}{l}\text { Blinding? } \\
\text { Overall survival }\end{array}$ & Yes & $\begin{array}{l}\text { Patients not blinded. No information } \\
\text { about blinding of the assessor. This is } \\
\text { judged not to be a source of bias for overall } \\
\text { survival. }\end{array}$ \\
\hline $\begin{array}{l}\text { Blinding? } \\
\text { Other outcomes }\end{array}$ & Unclear & $\begin{array}{l}\text { Patients and physicians not blinded. No in- } \\
\text { formation about blinding of the assessor. }\end{array}$ \\
\hline $\begin{array}{l}\text { Incomplete outcome data addressed? } \\
\text { All outcomes }\end{array}$ & Unclear & $\begin{array}{l}12 \text { years OS and tumour control outcome: } \\
20 / 327 \text { missing from the outcome analysis; } \\
\text { no information per study arm. The authors } \\
\text { do not give any further information about } \\
\text { the method of analysis (e.g. intention-to- } \\
\text { treat) } \\
\text { We do not believe that these few missing } \\
\text { patients induced large bias in the analysis, } \\
\text { but because the information is not available } \\
\text { by study arm and it remained unclear in } \\
\text { which arm patients who did not receive ra- } \\
\text { diotherapy were evaluated, we judged un- } \\
\text { clear. } \\
\text { For subgroup analysis this trial was consid- } \\
\text { ered to have no ITT analysis. }\end{array}$ \\
\hline Free of selective reporting? & Unclear & $\begin{array}{l}\text { In the methods section: "Disease-free sur- } \\
\text { vival was calculated for CR patients from } \\
\text { the beginning of treatment until clinical } \\
\text { or radiological and biopsy proven relapse." } \\
\text { No information about patients that did not } \\
\text { achieve CR. However, the denominator in } \\
\text { the results section is the full population, not } \\
\text { only patients in CR. Both disease-free sur- } \\
\text { vival and relapse free survival were calcu- } \\
\text { lated but only disease free survival was re- } \\
\text { ported. Due to the information given about } \\
\text { toxic deaths, overall survival and disease- } \\
\text { free survival, we assume that relapse free }\end{array}$ \\
\hline
\end{tabular}


Mexico B2H031 (Continued)

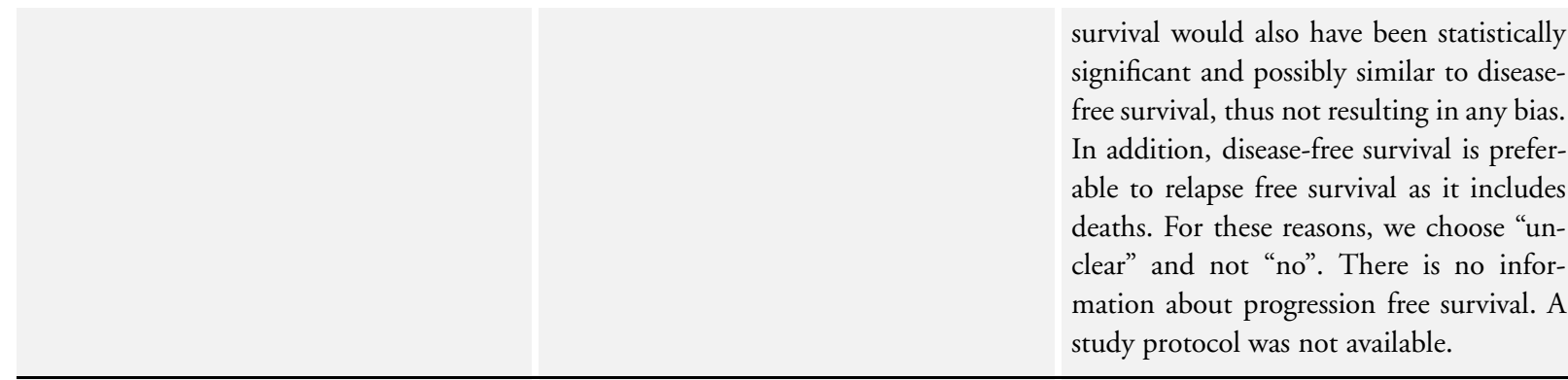

\section{MSKCC trial \#90-44}

Methods

Interventions
Participants

- A randomised controlled with two arms: CT-alone arm and CMT arm.

- Conducted by MSKCC (Memorial Sloan-Kettering Cancer Center), USA.

- Recruitment period from May 1990 to June 2000.

- 152 patients randomised.

- 152 patients evaluated for OS; 138 patients evaluated for response rate.

- 14 patients not evaluated for response outcome.

- Baseline patient's characteristics described.

- Median follow-up time 67 months (range 1 to 125 months).

- ITT analysis for overall survival; not ITT analysis for response outcomes.

- Inclusion criteria: patients with a confirmed diagnosis of Hodgkin lymphoma, without previous treatment and with clinical or pathological stage IA, IIA, IIB or IIIA and lack of bulky nodal tumour (mediastinal mass $\leq 0.33$, the thoracic diameter on chest x-ray measured at T11, and/or peripheral or retroperitoneal adenopathy $\leq 10 \mathrm{~cm}$ in its largest diameter).

- Exclusion criteria: patients with chronic lung disease with a diffusing capacity of less than $60 \%$ and/or with cardiac disease with clinical congestive heart failure or an abnormal ventricular ejection fraction $(<50 \%)$ on echocardiogram or multiple gated acquisition scan were excluded.

- CS I, II: CT-alone: 19, 46; CMT: 9, 58.

- CS III: CT-alone: 11; CMT: 9.

- Prognostic features not reported.

- Median age: CT-alone: 33 years (range 16-68 years); CMT: 39 years (range 15-66 years).

- Gender: 87 males, 65 females.

- Small imbalance in the distribution of sex, stage and histology, regarding baseline patient's characteristics in comparison arms.

- Histopathologic diagnosis: according to the Rye modification of the Lukes and Butler classification.

- CT-alone: 6 cycles of ABVD (doxorubicin $\left(25 \mathrm{mg} / \mathrm{m}^{2}\right)$, bleomycin $\left(10\right.$ units $\left./ \mathrm{m}^{2}\right)$, vinblastine $\left(6 \mathrm{mg} / \mathrm{m}^{2}\right)$, dacarbazine $\left(375 \mathrm{mg} / \mathrm{m}^{2}\right.$ intravenously, days 1 and 15$\left.)\right)$; repeated after every 28 days.

- CMT: same CT with extended-field radiotherapy (EF-RT) or involved-field radiotherapy (IF-RT); dose of RT 36 Gy in 180 cGY daily fractions starting after 4-6 
MSKCC trial \#90-44 (Continued)

weeks after completion of CT.

- Additional intervention: filgrastim was used for subsequent treatment of neutropenic patients.

\begin{tabular}{ll}
\hline Outcomes & - Overall survival reported; observation time 5 years. \\
& - Complete response reported. \\
& - Partial response reported. \\
& - Progression-free survival not reported. \\
& - Time to progression reported (see Table 3). \\
\hline \multirow{2}{*}{ Notes } & - $13 \%$ patients with CS IIIA. \\
& - Academic funding. \\
\hline
\end{tabular}

\section{Risk of bias}

\begin{tabular}{|c|c|c|}
\hline Item & Authors' judgement & Description \\
\hline Adequate sequence generation? & Yes & $\begin{array}{l}\text { Sequence generation was not reported. } \\
\text { "Randomisation was performed after a } \\
\text { check for eligibility. Patients were stratified } \\
\text { according to clinical stage (IA or IIa, IIIA, } \\
\text { I B or IIB)." } \\
\text { Presumably the randomisation was ade- } \\
\text { quate. }\end{array}$ \\
\hline Allocation concealment? & Yes & $\begin{array}{l}\text { "Patients were enrolled by telephone call or } \\
\text { fax to the MSKCC Clinical Trials Office" }\end{array}$ \\
\hline $\begin{array}{l}\text { Blinding? } \\
\text { Overall survival }\end{array}$ & Yes & $\begin{array}{l}\text { Patients and physicians not blinded. No in- } \\
\text { formation about blinding of the assessor. } \\
\text { This is judged not to be a source of bias for } \\
\text { overall survival. }\end{array}$ \\
\hline $\begin{array}{l}\text { Blinding? } \\
\text { Other outcomes }\end{array}$ & Unclear & $\begin{array}{l}\text { Patients and physicians not blinded. No in- } \\
\text { formation about blinding of the assessor. }\end{array}$ \\
\hline $\begin{array}{l}\text { Incomplete outcome data addressed? } \\
\text { All outcomes }\end{array}$ & Yes & $\begin{array}{l}11 \text { patients randomised to RT never re- } \\
\text { ceived RT: } 6 \text { refused, } 4 \text { progressed on che- } \\
\text { motherapy prior to receiving RT, } 1 \text { never } \\
\text { received RT because of bleomycin induced } \\
\text { toxicity to RT. } \\
\text { OS: all patients included in the analysis, } \\
\text { ITT-analysis } \\
\text { Tumour control: all patients included in } \\
\text { the analysis, ITT-analysis } \\
\text { Response rates: } 7 / 76 \text { excluded from CT- } \\
\text { alone arm and } 7 / 76 \text { excluded from CMT } \\
\text { arm; three lost to follow-up before comple- } \\
\text { tion of six cycles of chemotherapy and } 11\end{array}$ \\
\hline
\end{tabular}




\begin{tabular}{|c|c|c|}
\hline & & $\begin{array}{l}\text { stage IA patients with no measurable dis- } \\
\text { ease prior to treatment. }\end{array}$ \\
\hline Free of selective reporting? & Unclear & $\begin{array}{l}\text { Choice of progression outcome not de- } \\
\text { scribed - both disease-free survival and free- } \\
\text { dom from progression evaluated; freedom } \\
\text { from progression was closer to our defini- } \\
\text { tion of PFS and was thus used in the anal- } \\
\text { yses. }\end{array}$ \\
\hline Free of other bias? & Yes & No other bias identified. \\
\hline
\end{tabular}

\section{Characteristics of excluded studies [ordered by study ID]}

\begin{tabular}{|c|c|}
\hline Study & Reason for exclusion \\
\hline Andrieu 1999 & $\begin{array}{l}\text { Comparison arms not treated with CT-alone or CMT; all included patients received CMT. } \\
\text { Less than } 80 \% \text { of the participants had early stage Hodgkin lymphoma; only } 25 \% \text { of the included patients had } \\
\text { early stage Hodgkin lymphoma }\end{array}$ \\
\hline Bonnet 2007 & $\begin{array}{l}\text { Less than } 80 \% \text { of the participants had early stage Hodgkin lymphoma; only } 6 \text { of the } 576 \text { included patients } \\
\text { had Hodgkin lymphoma. }\end{array}$ \\
\hline Brusamolino 1994 & Comparison arms not treated with CT-alone or CMT; compared interventions RT-alone versus CMT. \\
\hline Cheveresan 1998 & Comparison arms not treated with CT-alone or CMT; all included patients received CMT. \\
\hline Cimino 1990 & Not a randomised controlled trial; a review article. \\
\hline Cosset 1992 & Not a randomised controlled trial; a review article. \\
\hline Desablens 1999 & Comparison arms not treated with CT-alone or CMT; all patients received CMT. \\
\hline Dionet 1988 & Comparison arms not treated with CT-alone or CMT and different CT regimens used in comparison arms. \\
\hline Hirsch 1996 & $\begin{array}{l}\text { Evaluation of pulmonary symptoms in patients randomised to MSKCC trials } 1989 \text { to } 1993 . \\
\text { Not a report of one specific trial } \\
\text { Relevant patients presumably analysed in MSKCC trial \#90-44 (recruitment 1990-2000) } \\
\text { Only } 45 \text { patients with the relevant comparison included } \\
\text { 30: } 6 \text { X ABVD } \\
\text { 15: } 6 \text { X ABVD plus EF RT } \\
\text { No mortality data given } \\
\text { Adverse events included only pulmonary function and included } 15 \text { patients not in the relevant randomised } \\
\text { comparison. } \\
\text { During CT } 53 \% \text { of patients had symptoms of cough or dyspnoea on exertion } \\
\text { At the end of follow-up ( }-1 \text { year after treatment), } 18 \% \text { (CT) vs. } 30 \% \text { (CMT) reported persistent symptoms }\end{array}$ \\
\hline
\end{tabular}


(Continued)

$(\mathrm{P}=0.36)$ Horning $1996 \begin{aligned} & \text { Less than } 80 \% \text { of the participants had early stage Hodgkin lymphoma; only } 42 \% \text { of the included patients had } \\ & \text { early stage Hodgkin lymphoma. }\end{aligned}$

Horning 2007 Comparison arms not treated with CT-alone or CMT; compared interventions RT-alone versus CMT.

\begin{tabular}{ll}
\hline Kim 2003 & Not a randomised controlled trial; a retrospective data analysis of patients' records with Hodgkin lymphoma. \\
\hline Koerholz 2004 & Not a randomised controlled trial. \\
\hline Kung 1993 & $\begin{array}{l}\text { Less than } 80 \% \text { of the participants had early stage Hodgkin lymphoma; 69\% of the included patients had early } \\
\text { stage Hodgkin lymphoma. No subgroup information available. (See also Kung 2006). }\end{array}$ \\
\hline Kung 2006 & $\begin{array}{l}\text { Less than } 80 \% \text { of the participants had early stage Hodgkin lymphoma; } 69 \% \text { of the included patients had early } \\
\text { stage Hodgkin lymphoma. No subgroup information available. }\end{array}$ \\
\hline Laskar 2004 & $\begin{array}{l}\text { Less than } 80 \% \text { of the participants had early stage Hodgkin lymphoma; } 55 \% \text { of the included patients had early } \\
\text { stage Hodgkin lymphoma. }\end{array}$
\end{tabular}

Longo $1992 \quad$ Not a randomised controlled trial; a review article about the trials (GATLA 9-H-77; O'Dwyer 1985).

\begin{tabular}{ll}
\hline Meyer 2005 & Chemotherapy differed between treatment arms (2 cycles of ABVD+RT vs. 4-6 cycles of ABVD) \\
\hline Nachman 2002 & $\begin{array}{l}\text { Less than 80\% of the participants had early stage Hodgkin lymphoma; 55\% of the included patients had early } \\
\text { stage Hodgkin lymphoma. }\end{array}$ \\
\hline Noordijk 2006 & Comparison arms not treated with CT-alone or CMT; compared interventions RT-alone versus CMT. \\
\hline O’Dwyer 1984 & $\begin{array}{l}\text { Less than 80\% of the participants with early stage Hodgkin lymphoma; } 69 \% \text { of the evaluable patients with } \\
\text { early stage Hodgkin lymphoma. Duplicate publication (see also O' Dwyer } 1985) .\end{array}$ \\
\hline
\end{tabular}

O’Dwyer 1985 Less than $80 \%$ of the participants had early stage Hodgkin lymphoma; $69 \%$ of the evaluable patients had early stage Hodgkin lymphoma.

\begin{tabular}{ll}
\hline Pavlovsky 1997 & Comparison arms not treated with CT-alone or CMT. \\
\hline Picardi 2007 & $\begin{array}{l}\text { Less than 80\% of the participants had early stage Hodgkin lymphoma; 66\% of the included patients had early } \\
\text { stage Hodgkin lymphoma. No subgroup information available. }\end{array}$ \\
\hline Radford 2002 & Comparison arms not treated with CT-alone or CMT; compared interventions RT-alone versus CMT. \\
\hline Rüffer 1996 & Comparison arms not treated with CT-alone or CMT; compared interventions RT versus RT. \\
\hline Rüffer 1998 & Comparison arms not treated with CT-alone or CMT; all patients received CMT. \\
\hline Rüffer 1999 & $\begin{array}{l}\text { Comparison arms not treated with CT-alone or CMT; all patients received CMT. Duplicate publication (see } \\
\text { also Ruffer 1998); all patients received CMT. }\end{array}$
\end{tabular}




Specht $1992 \quad$ Not a randomised controlled trial; a review article.

Straus $1989 \quad$ Comparison arms not treated with CT-alone or CMT; all patients received CMT.

Thistlethwaite 2007 Comparison arms not treated with CT-alone or CMT; compared interventions RT-alone versus CMT.

\section{Characteristics of ongoing studies [ordered by study ID]}

\section{EORTC-GELA HD 10}

Trial name or title Official title: The H10 EORTC/GELA Randomized Intergroup Trial on Early FDG-PET Scan Guided Treatment Adaptation Versus Standard Combined Modality Treatment in Patients With Supradiaphragmatic Stage I/II Hodgkin's Lymphoma

\begin{tabular}{|c|c|}
\hline Methods & Randomised controlled trial \\
\hline Participants & $\begin{array}{l}\text { - } 15 \text { Years to } 70 \text { Years } \\
\circ \text { Histologically confirmed Hodgkin's lymphoma } \\
\circ \text { No nodular lymphocyte-predominant subtype (nodular paragranuloma) } \\
\circ \text { Supradiaphragmatic Ann Arbor clinical stage I or II disease } \\
\circ \text { Must meet criteria for } 1 \text { of the following prognostic subsets: } \\
\quad \diamond \text { Unfavourable subset, defined as meeting } 1 \text { of the following criteria: } \\
\text { Clinical stage II disease with }>4 \text { nodal areas involved } \\
\text { Mediastinum and hili are considered as } 1 \text { nodal area } \\
\text { Age }>50 \text { years } \\
\text { Erythrocyte sedimentation rate (ESR) }<50 \mathrm{~mm} / \mathrm{hr} \text { with no B symptoms ESR }<30 \mathrm{~mm} / \mathrm{hr} \text { with B } \\
\text { symptomsMediastinum/thoracic (MT) ratio }<0.35 \\
\quad \diamond \text { Favourable subset, defined as meeting all of the following criteria: } \\
\text { Clinical stage I disease OR stage II disease with }<3 \text { involved areas } \\
\text { Age }<50 \text { years } \\
\text { ESR }<50 \text { mm/hr (no B symptoms) OR ESR }<30 \mathrm{~mm} / \mathrm{hr} \text { (B symptoms present) } \\
\text { MT ratio }<0.35 \\
\circ \text { Previously untreated disease } \\
\circ \text { Planning to undergo fludeoxyglucose F } 18 \text { positron emission tomography after the first } 2 \text { courses } \\
\text { of study chemotherapy } \\
\text { - PATIENT CHARACTERISTICS:WHO performance status } 0-3 \\
\circ \text { Bilirubin }<2.5 \text { times upper limit of normal (ULN) } \\
\circ \text { Creatinine }<2.5 \text { times ULN } \\
\circ \text { Not pregnant or nursing } \\
\circ \text { Negative pregnancy test } \\
\circ \text { Fertile patients must use effective contraception } \\
\circ \text { No severe cardiac, pulmonary, neurologic, psychiatric, or metabolic disease } \\
\circ \text { No unstable diabetes mellitus } \\
\circ \text { No other malignancies within the past } 5 \text { years except for basal cell skin cancer or adequately } \\
\text { treated carcinoma in situ of the cervix } \\
\circ \text { No known HIV infection }\end{array}$ \\
\hline
\end{tabular}


EORTC-GELA HD 10 (Continued)

\begin{tabular}{|c|c|}
\hline & $\begin{array}{l}\circ \text { No psychological, familial, sociological, or geographical condition that would preclude study } \\
\text { compliance }\end{array}$ \\
\hline Interventions & $\begin{array}{l}\text { - Arm I (standard): Patients receive ABVD chemotherapy comprising doxorubicin hydrochloride IV, } \\
\text { bleomycin IV or intramuscularly (IM), vinblastine IV, and dacarbazine IV on days } 1 \text { and } 15 \text {. Treatment } \\
\text { repeats every } 28 \text { days in the absence of disease progression or unacceptable toxicity. Patients with favourable } \\
\text { prognostic profile receive } 3 \text { courses of ABVD. Patients with unfavourable prognostic profile receive } 4 \\
\text { courses of ABVD. Patients undergo FDG-PET scan after completion of } 2 \text { courses of ABVD. Beginning 3-4 } \\
\text { weeks after completion of ABVD, patients undergo involved-node radiotherapy (INRT) } 5 \text { days a week for } \\
\text { 4-6 weeks. } \\
\text { - Arm II (experimental): Patients receive ABVD as in arm I for } 2 \text { courses and then undergo FDG-PET } \\
\text { scan. Further treatment is adapted according to FDG-PET scan result.FDG-PET negative: Patients with } \\
\text { favourable prognostic profile receive } 2 \text { additional courses of ABVD. Patients with unfavourable prognostic } \\
\text { profile receive } 4 \text { additional courses of ABVD. FDG-PET positive: Patients receive escalated BEACOPP } \\
\text { chemotherapy comprising cyclophosphamide IV and doxorubicin hydrochloride IV on day 1, vincristine IV } \\
\text { and bleomycin IV or IM on day } 8 \text {, etoposide IV on days } 1-3 \text {, oral procarbazine hydrochloride on days } 1-7 \text {, } \\
\text { oral prednisone on days } 1-14 \text {, and filgrastim (G-CSF) subcutaneously beginning on day } 9 \text { and continuing } \\
\text { until blood count recover. Treatment repeats every } 21 \text { days for } 2 \text { courses in the absence of disease } \\
\text { progression or unacceptable toxicity. Beginning } 3-4 \text { weeks after completion of BEACOPP, patients undergo } \\
\text { INRT } 5 \text { days a week for } 4-6 \text { weeks. }\end{array}$ \\
\hline Outcomes & $\begin{array}{l}\text { Primary Outcome Measures: Progression-free survival } \\
\text { Secondary Outcome Measures: Event-free survival; Overall survival; Long-term toxicity, in terms of secondary } \\
\text { malignancies, cardiovascular events, and pulmonary events; Response } \\
\text { Duration } 10 \text { years }\end{array}$ \\
\hline Starting date & October 2006 \\
\hline Contact information & J.Raemaekers@hemat.umcn.nl \\
\hline Notes & NCT00433433, 1600 patients planned \\
\hline
\end{tabular}

\section{Friedman}

Trial name or title Official title: A phase III group-wide study of dose-intensive response-based chemotherapy and radiation therapy for children and adolescents with newly diagnosed intermediate risk Hodgkin disease.

A randomised controlled trials.
Initial CT to all patients with 2 cycles of ABVE-PC (doxorubicin, bleomycin, vincristine, etoposide, pred-
nisone, cyclophosphamide).
Patients with RER (rapid early response) that > $60 \%$ disease reduction receive 2 additional courses of ABVE-
PC.
Patients with complete response after 4 courses randomised to receive either RT or no further treatment.
Patients with SER (slow early response) that < $60 \%$ disease reduction: Arm I: 2 courses of DECC (dexam-
ethasone, etoposide, cytarabine, cisplatin) and 2 additional courses of ABVE-PC and RT; Arm II: Patients
with SER receive 2 additional courses of ABVE-PC without DECC and RT (Arm II). These patients are not
relevant for the review




\section{Friedman (Continued)}

\begin{tabular}{ll}
\hline Participants & $\begin{array}{l}\text { Both male and female patients up to } 21 \text { years with newly diagnosed Hodgkin's lymphoma without prior } \\
\text { therapy. }\end{array}$ \\
\hline Interventions & $\begin{array}{l}\text { CT-alone: } 4 \text { cycles of ABVE-PC, repeated after every } 21 \text { days. } \\
\text { CMT: } 4 \text { cycles of ABVE-PC and RT }\end{array}$ \\
\hline Outcomes & $\begin{array}{l}\text { Primary outcome measure: event-free survival. } \\
\text { Secondary outcome measures: overall survival; disease response; toxicity. }\end{array}$ \\
\hline Starting date & September 2002. \\
\hline Contact information & $\begin{array}{l}\text { Investigator: Debra L. Friedman MD, Childrens Hospital and Regional Medical Center, Seattle. Childrens } \\
\text { Oncology Group, National Cancer Institute (NCI). }\end{array}$ \\
\hline Notes & $\begin{array}{l}\text { The trial has been suspended (last accessed August 7th, 2009) } \\
\text { Final data collection date for primary outcome measure June 2010. } \\
\text { Clinical Trials.gov Identifier: NCT00025259 (www.clinicaltrials.gov) }\end{array}$ \\
\hline
\end{tabular}

\section{GHSG HD 16}

\begin{tabular}{l|l} 
Trial name or title & $\begin{array}{l}\text { Official Title: HD16 for Early Stages - Treatment Optimization Trial in the First-Line Treatment of Early } \\
\text { Stage Hodgkin Lymphoma; Treatment Stratification by Means of FDG-PET }\end{array}$ \\
\hline Methods & Randomised controlled trial, non-inferiority design \\
\hline Participants & $\begin{array}{l}\text { 18 Years to } 75 \text { Years } \\
\text { Inclusion Criteria: } \\
\text { - Hodgkin lymphoma } \\
\text { - CS I, II without any of the following risk factors: large mediastinal mass }(>1 / 3 \text { of maximum transverse } \\
\text { thorax diameter) extranodal involvement elevated ESR3 or more involved nodal areas } \\
\text { Exclusion Criteria: } \\
\text { - Weucocytes }<3000 / \mu \mathrm{l} \\
\text { - Platelets }<100000 / \mu \mathrm{l} \\
\text { - Hodgkin lymphoma as composite lymphoma } \\
\text { - Activity index }(\mathrm{WHO})>2\end{array}$ \\
\hline
\end{tabular}

Interventions Arm 1: 2 cycles ABVD followed by 30 Gy IF-RT irrespective of FDG-PET results after chemotherapy Arm 2: 2 cycles ABVD followed by 30 Gy IF-RT if FDG-PET is positive after chemotherapy; 2 cycles ABVD and treatment stop if FDG-PET is negative after chemotherapy

Outcomes $\quad$ Primary Outcome Measures: Progression Free Survival (Time Frame: 5 years)

Secondary Outcome Measures: Overall survival, acute and late toxicity, CR-rate (Time Frame: 5 years)

\begin{tabular}{ll} 
Starting date & unclear \\
\hline Contact information & Michael Fuchs; GHSG@uk-koeln.de
\end{tabular}


GHSG HD 16 (Continued)

Notes clinicaltrials.gov identifier NCT00736320; 1100 patients to be enrolled 
DATA AND ANALYSES

\section{Comparison 1. Overall Survival}

\begin{tabular}{|c|c|c|c|c|}
\hline Outcome or subgroup title & $\begin{array}{l}\text { No. of } \\
\text { studies }\end{array}$ & $\begin{array}{c}\text { No. of } \\
\text { participants }\end{array}$ & Statistical method & Effect size \\
\hline 1 All trials & 5 & 1245 & Hazard Ratio (Random, 95\% CI) & $0.40[0.27,0.61]$ \\
\hline $\begin{array}{l}2 \text { Excluding the trial with highest } \\
\text { weight (Aviles) }\end{array}$ & 4 & 1044 & Hazard Ratio (Random, 95\% CI) & $0.57[0.33,0.98]$ \\
\hline $\begin{array}{l}3 \text { Proportion of patients early } \\
\text { favourable }\end{array}$ & 5 & & Hazard Ratio (Random, 95\% CI) & $0.40[0.27,0.61]$ \\
\hline $\begin{array}{l}3.1 \text { All patients early } \\
\text { favourable }\end{array}$ & 1 & & Hazard Ratio (Random, 95\% CI) & $0.27[0.04,1.74]$ \\
\hline $\begin{array}{l}3.2-1 / 3 \text { to } 1 / 2 \text { patients early } \\
\text { unfavourable }\end{array}$ & 2 & & Hazard Ratio (Random, 95\% CI) & $0.57[0.28,1.15]$ \\
\hline $\begin{array}{l}3.3 \text { All patients early } \\
\text { unfavourable }\end{array}$ & 2 & & Hazard Ratio (Random, 95\% CI) & $0.31[0.18,0.54]$ \\
\hline 4 Bulky vs non-bulky & 3 & & Hazard Ratio (Random, 95\% CI) & $0.29[0.18,0.48]$ \\
\hline 4.1 Bulky disease & 1 & & Hazard Ratio (Random, 95\% CI) & $0.29[0.17,0.51]$ \\
\hline 4.2 Non-bulky disease & 2 & & Hazard Ratio (Random, 95\% CI) & $0.30[0.10,0.87]$ \\
\hline 5 Timing of radiotherapy & 5 & & Hazard Ratio (Random, 95\% CI) & $0.40[0.27,0.61]$ \\
\hline $\begin{array}{l}5.1 \text { Radiotherapy after } \\
\text { chemotherapy }\end{array}$ & 2 & & Hazard Ratio (Random, 95\% CI) & $0.30[0.10,0.87]$ \\
\hline $\begin{array}{l}5.2 \text { Sandwich technique } \\
\text { (CT-RT-CT) }\end{array}$ & 2 & & Hazard Ratio (Random, 95\% CI) & $0.44[0.19,1.04]$ \\
\hline $\begin{array}{l}5.3 \text { Chemotherapy after } \\
\text { radiotherapy }\end{array}$ & 1 & & Hazard Ratio (Random, 95\% CI) & $0.63[0.11,3.65]$ \\
\hline 6 Type of radiotherapy & 5 & & Hazard Ratio (Random, 95\% CI) & $0.40[0.27,0.61]$ \\
\hline 6.1 Involved field & 3 & & Hazard Ratio (Random, 95\% CI) & $0.62[0.33,1.17]$ \\
\hline 6.2 Extended field & 1 & & Hazard Ratio (Random, 95\% CI) & $0.29[0.17,0.51]$ \\
\hline 6.3 Mixed & 1 & & Hazard Ratio (Random, 95\% CI) & $0.31[0.08,1.15]$ \\
\hline 7 Type of chemotherapy & 5 & & Hazard Ratio (Random, 95\% CI) & $0.40[0.27,0.59]$ \\
\hline $7.1 \mathrm{ABVD}$ & 2 & & Hazard Ratio (Random, 95\% CI) & $0.30[0.18,0.50]$ \\
\hline 7.2 CVPP & 2 & & Hazard Ratio (Random, 95\% CI) & $0.68[0.35,1.31]$ \\
\hline 7.3 EBVP & 1 & & Hazard Ratio (Random, 95\% CI) & $0.27[0.04,1.73]$ \\
\hline 8 Length of follow-up & 5 & & Hazard Ratio (Random, 95\% CI) & $0.40[0.27,0.61]$ \\
\hline 8.1 Six years or less & 4 & & Hazard Ratio (Random, 95\% CI) & $0.55[0.31,0.96]$ \\
\hline 8.2 More than six years & 1 & & Hazard Ratio (Random, 95\% CI) & $0.29[0.17,0.51]$ \\
\hline 94 year survival in the CT group & 5 & & Hazard Ratio (Random, 95\% CI) & $0.40[0.27,0.61]$ \\
\hline $9.1>90 \%$ & 1 & & Hazard Ratio (Random, 95\% CI) & $0.27[0.04,1.74]$ \\
\hline $9.280 \%-90 \%$ & 3 & & Hazard Ratio (Random, 95\% CI) & $0.41[0.22,0.77]$ \\
\hline 9.3 unknown & 1 & & Hazard Ratio (Random, 95\% CI) & $0.63[0.11,3.65]$ \\
\hline 10 Allocation concealment & 5 & & Hazard Ratio (Random, 95\% CI) & $0.40[0.27,0.61]$ \\
\hline $\begin{array}{l}\text { 10.1 Allocation concealment } \\
\text { unclear }\end{array}$ & 3 & & Hazard Ratio (Random, 95\% CI) & $0.46[0.23,0.89]$ \\
\hline $\begin{array}{l}10.2 \text { Allocation concealment } \\
\text { adequate }\end{array}$ & 2 & & Hazard Ratio (Random, 95\% CI) & $0.30[0.10,0.87]$ \\
\hline 11 ITT-analysis & 5 & & Hazard Ratio (Random, 95\% CI) & $0.40[0.27,0.61]$ \\
\hline 11.1 ITT-analysis & 2 & & Hazard Ratio (Random, 95\% CI) & $0.30[0.10,0.87]$ \\
\hline
\end{tabular}

Chemotherapy alone versus chemotherapy plus radiotherapy for early stage Hodgkin lymphoma (Review)

Copyright (@) 20II The Cochrane Collaboration. Published by John Wiley \& Sons, Ltd. 


\section{Comparison 2. Progression-Free Survival}

\begin{tabular}{|c|c|c|c|c|}
\hline Outcome or subgroup title & $\begin{array}{l}\text { No. of } \\
\text { studies }\end{array}$ & $\begin{array}{c}\text { No. of } \\
\text { participants }\end{array}$ & Statistical method & Effect size \\
\hline 1 All trials & 4 & 1208 & Hazard Ratio (Random, 95\% CI) & $0.41[0.25,0.66]$ \\
\hline 2 Type of chemotherapy & 4 & & Hazard Ratio (Random, 95\% CI) & $0.41[0.25,0.66]$ \\
\hline $2.1 \mathrm{ABVD}$ & 2 & & Hazard Ratio (Random, 95\% CI) & $0.47[0.16,1.35]$ \\
\hline $2.2 \mathrm{CVPP}$ & 1 & & Hazard Ratio (Random, 95\% CI) & $0.55[0.35,0.86]$ \\
\hline $2.3 \mathrm{EBVP}$ & 1 & & Hazard Ratio (Random, 95\% CI) & $0.27[0.17,0.43]$ \\
\hline $\begin{array}{l}3 \text { Proportion of patients early } \\
\text { favourable }\end{array}$ & 4 & 429 & Hazard Ratio (Random, 95\% CI) & $0.41[0.25,0.66]$ \\
\hline $\begin{array}{l}3.1 \text { All patients early } \\
\text { favourable }\end{array}$ & 1 & 0 & Hazard Ratio (Random, 95\% CI) & $0.27[0.17,0.43]$ \\
\hline $\begin{array}{l}3.2 \text { mixed patient population } \\
(-1 / 3 \text { to } 1 / 2)\end{array}$ & 2 & 429 & Hazard Ratio (Random, 95\% CI) & $0.61[0.41,0.90]$ \\
\hline $\begin{array}{l}3.3 \text { All patients early } \\
\text { unfavourable }\end{array}$ & 1 & 0 & Hazard Ratio (Random, 95\% CI) & $0.29[0.17,0.48]$ \\
\hline 4 Definition of progression & 4 & & Hazard Ratio (Random, 95\% CI) & $0.41[0.25,0.66]$ \\
\hline 4.1 In CR patients & 1 & & Hazard Ratio (Random, 95\% CI) & $0.27[0.17,0.43]$ \\
\hline 4.2 Progress in all patients & 2 & & Hazard Ratio (Random, 95\% CI) & $0.61[0.41,0.90]$ \\
\hline 4.3 Unclear & 1 & & Hazard Ratio (Random, 95\% CI) & $0.29[0.17,0.48]$ \\
\hline 5 Timing of radiotherapy & 4 & & Hazard Ratio (Random, 95\% CI) & $0.41[0.25,0.66]$ \\
\hline $\begin{array}{l}5.1 \text { Radiotherapy after } \\
\text { chemotherapy }\end{array}$ & 2 & & Hazard Ratio (Random, 95\% CI) & $0.45[0.15,1.41]$ \\
\hline $\begin{array}{l}5.2 \text { Sandwich technique } \\
\text { (CT-RT-CT) }\end{array}$ & 2 & & Hazard Ratio (Random, 95\% CI) & $0.40[0.22,0.75]$ \\
\hline 6 Type of radiotherapy & 4 & & Hazard Ratio (Random, 95\% CI) & $0.41[0.25,0.66]$ \\
\hline 6.1 Involved field & 2 & & Hazard Ratio (Random, 95\% CI) & $0.38[0.19,0.78]$ \\
\hline 6.2 Extended field & 1 & & Hazard Ratio (Random, 95\% CI) & $0.29[0.17,0.48]$ \\
\hline 6.3 Mixed & 1 & & Hazard Ratio (Random, 95\% CI) & $0.85[0.37,1.94]$ \\
\hline 7 Length of follow-up & 4 & & Hazard Ratio (Random, 95\% CI) & $0.41[0.25,0.66]$ \\
\hline $\begin{array}{l}7.1 \text { Median follow-up } \leq 6 \\
\text { years }\end{array}$ & 3 & & Hazard Ratio (Random, 95\% CI) & $0.47[0.25,0.89]$ \\
\hline $\begin{array}{l}7.2 \text { Median follow-up > } 6 \\
\text { years }\end{array}$ & 1 & & Hazard Ratio (Random, 95\% CI) & $0.29[0.17,0.48]$ \\
\hline 8 Allocation concealment & 4 & & Hazard Ratio (Random, 95\% CI) & $0.41[0.25,0.66]$ \\
\hline $\begin{array}{l}8.1 \text { Allocation concealment } \\
\text { unclear }\end{array}$ & 2 & & Hazard Ratio (Random, 95\% CI) & $0.40[0.22,0.75]$ \\
\hline $\begin{array}{l}8.2 \text { Allocation concealment } \\
\text { adequate }\end{array}$ & 2 & & Hazard Ratio (Random, 95\% CI) & $0.45[0.15,1.41]$ \\
\hline 9 ITT-Analysis & 4 & & Hazard Ratio (Random, 95\% CI) & $0.41[0.25,0.65]$ \\
\hline 9.1 ITT-analysis & 2 & & Hazard Ratio (Random, 95\% CI) & $0.44[0.15,1.30]$ \\
\hline 9.2 No ITT-analysis & 2 & & Hazard Ratio (Random, 95\% CI) & $0.40[0.22,0.75]$ \\
\hline
\end{tabular}


Comparison 3. Complete Response Rate

\begin{tabular}{lccll} 
Outcome or subgroup title & $\begin{array}{c}\text { No. of } \\
\text { studies }\end{array}$ & $\begin{array}{c}\text { No. of } \\
\text { participants }\end{array}$ & Statistical method & Effect size \\
\hline 1 All trials & 4 & 653 & Risk Ratio (M-H, Random, 95\% CI) & $1.07[0.98,1.17]$ \\
2 Type of Chemotherapy & 4 & 653 & Risk Ratio (M-H, Random, 95\% CI) & $1.07[0.98,1.17]$ \\
$\quad 2.1$ CVPP & 2 & 314 & Risk Ratio (M-H, Random, 95\% CI) & $1.24[0.88,1.74]$ \\
2.2 ABVD & 2 & 339 & Risk Ratio (M-H, Random, 95\% CI) & $1.02[0.95,1.09]$ \\
3 ITT-analysis & 4 & 653 & Risk Ratio (M-H, Random, 95\% CI) & $1.07[0.98,1.17]$ \\
3.1 ITT-analysis & 1 & 138 & Risk Ratio (M-H, Random, 95\% CI) & $1.0[0.92,1.09]$ \\
3.2 no ITT-analysis & 3 & 515 & Risk Ratio (M-H, Random, 95\% CI) & $1.11[0.99,1.24]$ \\
4 Number of evaluable patients & 4 & 653 & Risk Ratio (M-H, Random, 95\% CI) & $1.07[0.98,1.17]$ \\
$\quad 4.1<10 \%$ not evaluated & 3 & 616 & Risk Ratio (M-H, Random, 95\% CI) & $1.05[0.99,1.11]$ \\
$4.2 \geq 10 \%$ not evaluated & 1 & 37 & Risk Ratio (M-H, Random, 95\% CI) & $1.55[1.06,2.27]$ \\
\hline
\end{tabular}

Comparison 4. Overall Response Rate

\begin{tabular}{lcccc} 
Outcome or subgroup title & $\begin{array}{c}\text { No. of } \\
\text { studies }\end{array}$ & $\begin{array}{c}\text { No. of } \\
\text { participants }\end{array}$ & Statistical method & Effect size \\
\hline 1 All Trials & 3 & 616 & Risk Ratio (M-H, Random, 95\% CI) & $1.00[0.96,1.04]$ \\
\hline
\end{tabular}

\section{Analysis I.I. Comparison I Overall Survival, Outcome I All trials.}

Review: Chemotherapy alone versus chemotherapy plus radiotherapy for early stage Hodgkin lymphoma

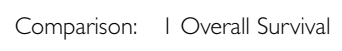

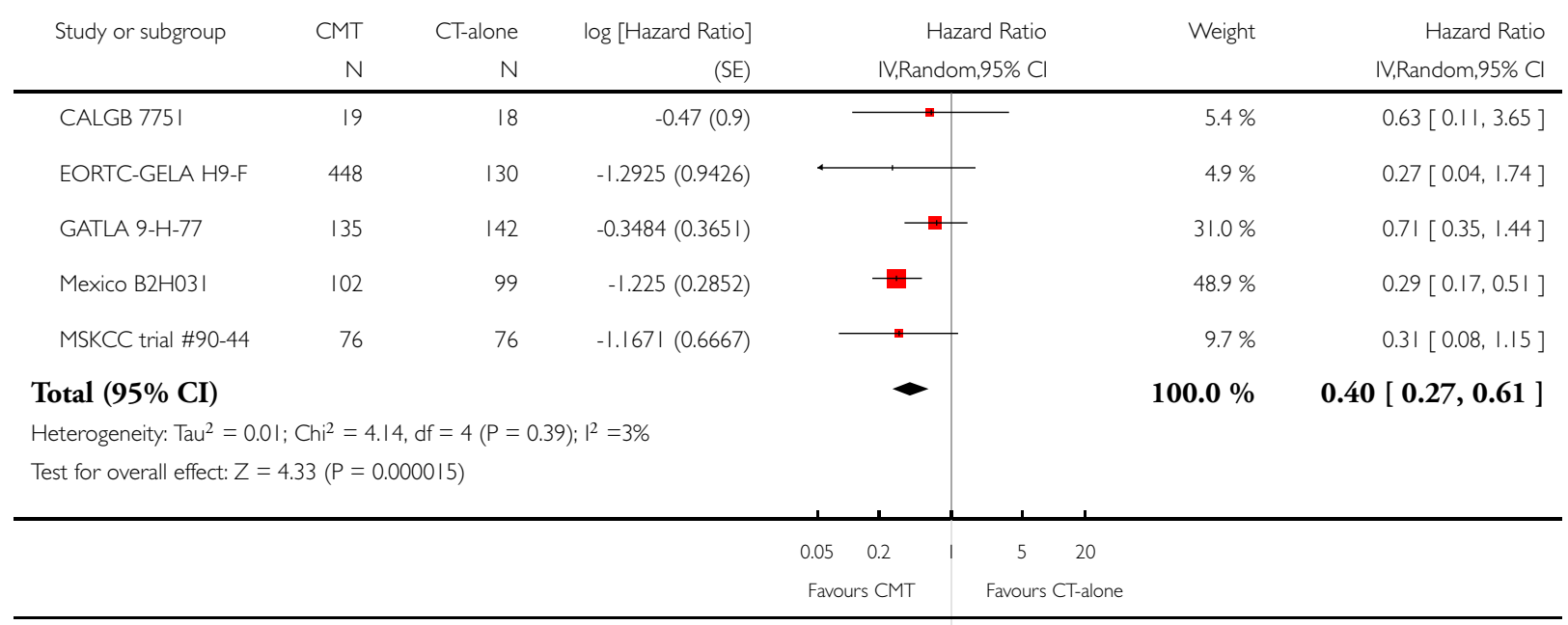


Analysis I.2. Comparison I Overall Survival, Outcome 2 Excluding the trial with highest weight (Aviles).

Review: Chemotherapy alone versus chemotherapy plus radiotherapy for early stage Hodgkin lymphoma

Comparison: I Overall Survival

Outcome: 2 Excluding the trial with highest weight (Aviles)

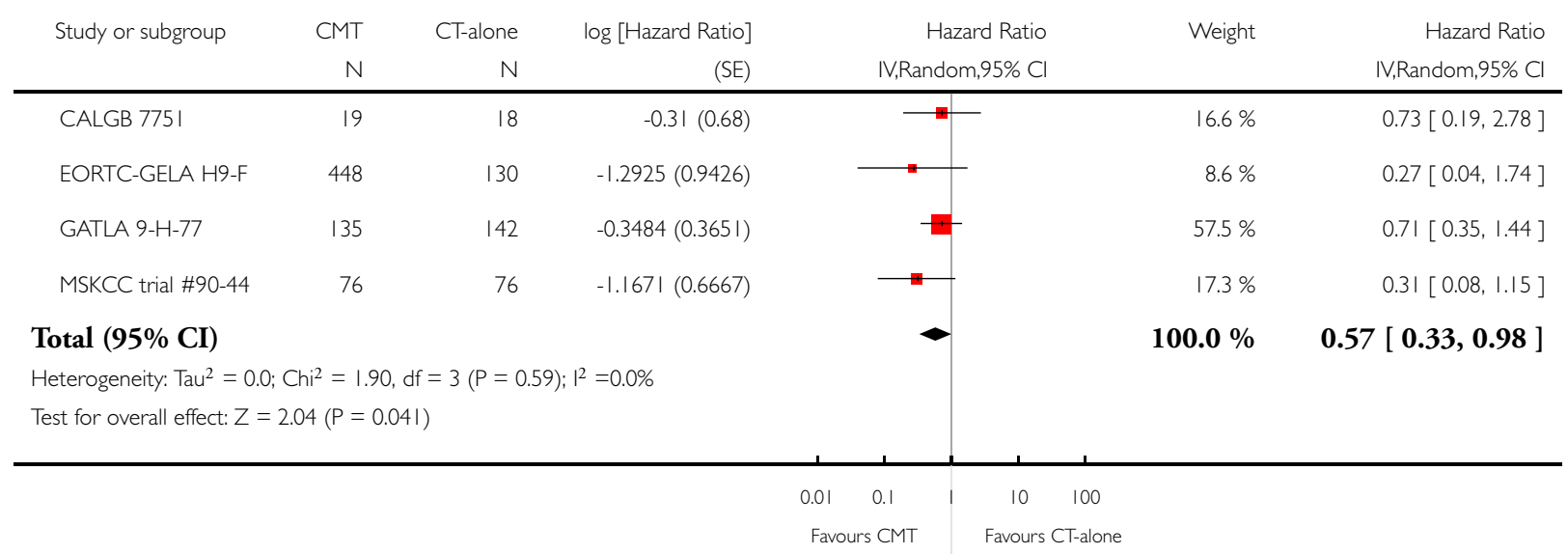




\section{Analysis I.3. Comparison I Overall Survival, Outcome 3 Proportion of patients early favourable.}

Review: Chemotherapy alone versus chemotherapy plus radiotherapy for early stage Hodgkin lymphoma

Comparison: I Overall Survival

Outcome: 3 Proportion of patients early favourable

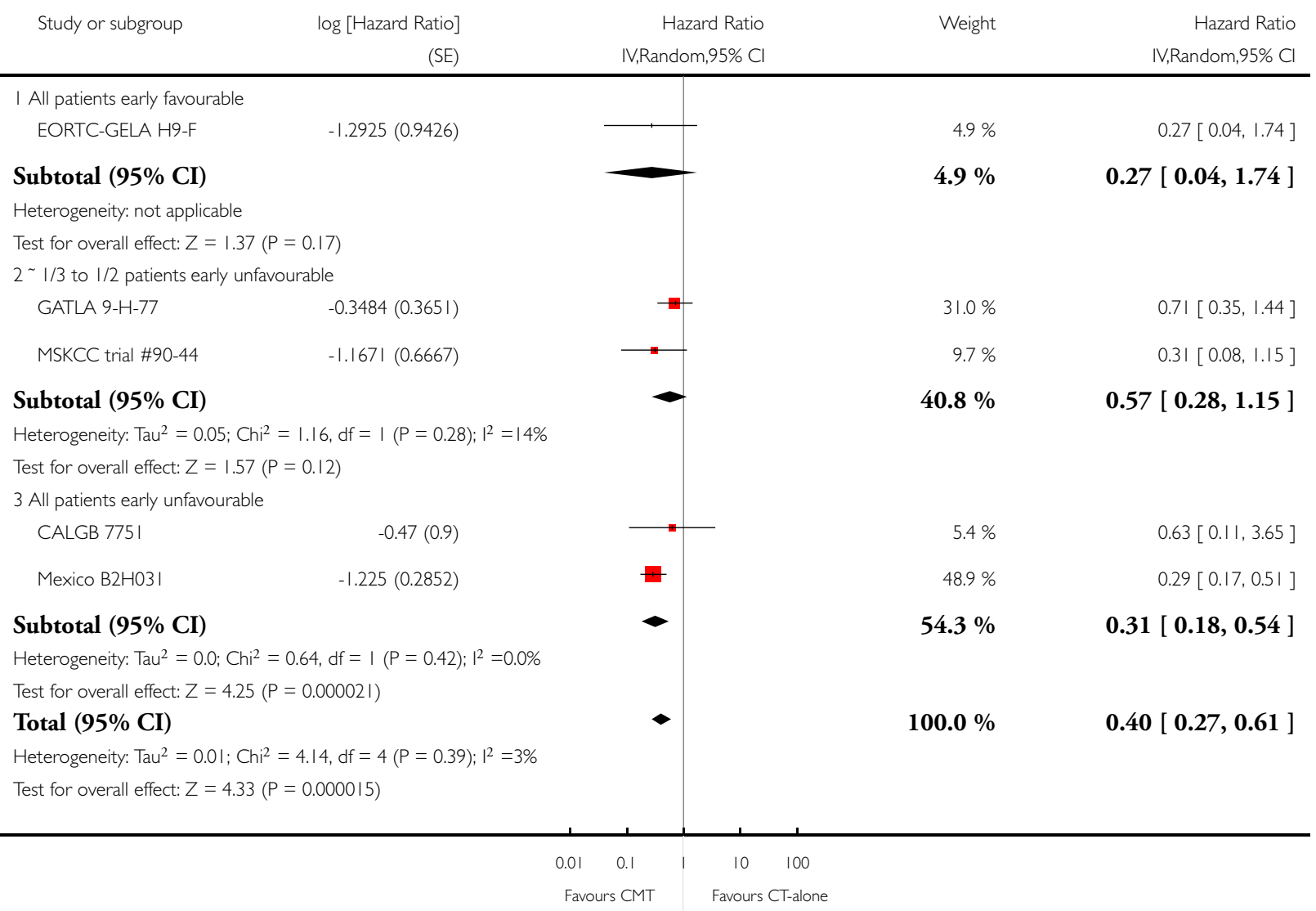




\section{Analysis I.4. Comparison I Overall Survival, Outcome 4 Bulky vs non-bulky.}

Review: Chemotherapy alone versus chemotherapy plus radiotherapy for early stage Hodgkin lymphoma

Comparison: I Overall Survival

Outcome: 4 Bulky vs non-bulky

Study or subgroup $\quad \log [$ Hazard Ratio

\section{Bulky disease}

Mexico $\mathrm{B} 2 \mathrm{HO} 3$

$-1.225(0.2852)$

Subtotal (95\% CI)

Heterogeneity: not applicable

Test for overall effect: $Z=4.30(P=0.000017)$

2 Non-bulky disease

$\begin{array}{lr}\text { EORTC-GELA H9-F } & -1.2925(0.9426) \\ \text { MSKCC trial \#90-44 } & -1.1671(0.6667)\end{array}$

Subtotal (95\% CI)

Heterogeneity: $\mathrm{Tau}^{2}=0.0 ; \mathrm{Chi}^{2}=0.0 \mathrm{I}, \mathrm{df}=\mathrm{I}(\mathrm{P}=0.9 \mathrm{I}) ; \mathrm{I}^{2}=0.0 \%$

Test for overall effect: $Z=2.22(P=0.026)$

Total (95\% CI)

Heterogeneity: $\mathrm{Tau}^{2}=0.0 ; \mathrm{Chi}^{2}=0.0 \mathrm{l}, \mathrm{df}=2(\mathrm{P}=0.99) ; \mathrm{I}^{2}=0.0 \%$

Test for overall effect: $Z=4.84(P<0.0000 I)$
Hazard Ratio

IV,Random,95\% Cl

Weight

Hazard Ratio IV,Random,95\% Cl
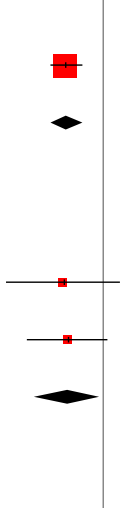

$-$

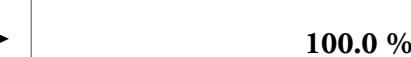

$100.0 \%$

$0.29[0.18,0.48]$

Favours CMT

$10 \quad 100$

Favours CT-alone 


\section{Analysis I.5. Comparison I Overall Survival, Outcome 5 Timing of radiotherapy.}

Review: Chemotherapy alone versus chemotherapy plus radiotherapy for early stage Hodgkin lymphoma

Comparison: I Overall Survival

Outcome: 5 Timing of radiotherapy

Study or subgroup $\quad \log$ [Hazard Ratio] Hazard Ratio Waight Ratio

(SE) IV,Random,95\% Cl

I Radiotherapy after chemotherapy

EORTC-GELA H9-F

$-1.2925(0.9426)$

$0.27[0.04,1.74]$

MSKCC trial \#90-44

$-1.167 \mid(0.6667)$

$4.9 \%$

$9.7 \%$

Subtotal (95\% CI)

Heterogeneity: $\mathrm{Tau}^{2}=0.0 ; \mathrm{Chi}^{2}=0.0 \mathrm{I}, \mathrm{df}=\mathrm{I}(\mathrm{P}=0.9 \mathrm{I}) ; \mathrm{I}^{2}=0.0 \%$

Test for overall effect: $Z=2.22(P=0.026)$

2 Sandwich technique (CT-RT-CT)

$\begin{array}{ll}\text { GATLA 9-H-77 } & -0.3484(0.365 \mathrm{I}) \\ & -1.225(0.2852)\end{array}$

Mexico B2HO3

$-1.225(0.2852)$

Subtotal (95\% CI)

Heterogeneity: $\mathrm{Tau}^{2}=0.28 ; \mathrm{Chi}^{2}=3.58, \mathrm{df}=\mathrm{I}(\mathrm{P}=0.06) ; \mathrm{I}^{2}=72 \%$

Test for overall effect: $Z=1.87(P=0.062)$

3 Chemotherapy after radiotherapy
CALGB 775।
$-0.47(0.9)$

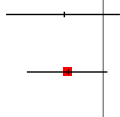

$14.6 \%$

$31.0 \%$

$0.71[0.35,1.44]$

$48.9 \%$

$0.29[0.17,0.51]$

$80.0 \%$

Subtotal (95\% CI)

Heterogeneity: not applicable

Test for overall effect: $Z=0.52(P=0.60)$

Total (95\% CI)

Heterogeneity: $\mathrm{Tau}^{2}=0.01 ; \mathrm{Chi}^{2}=4.14, \mathrm{df}=4(\mathrm{P}=0.39) ; \mathrm{I}^{2}=3 \%$

Test for overall effect: $Z=4.33(P=0.000015)$
$5.4 \%$

$0.63[0.11,3.65]$

$100.0 \%$

$0.40[0.27,0.61]$ 


\section{Analysis I.6. Comparison I Overall Survival, Outcome 6 Type of radiotherapy.}

\begin{tabular}{|c|c|c|c|c|}
\hline \multicolumn{5}{|c|}{ Comparison: I Overall Survival } \\
\hline \multicolumn{5}{|c|}{ Outcome: 6 Type of radiotherapy } \\
\hline \multirow[t]{2}{*}{ Study or subgroup } & $\log$ [Hazard Ratio] & Hazard Ratio & Weight & Hazard Ratio \\
\hline & (SE) & IV,Random,95\% Cl & & IV,Random,95\% Cl \\
\hline \multicolumn{5}{|l|}{ | Involved field } \\
\hline CALGB 7751 & $-0.47(0.9)$ & $\longrightarrow$ & $5.4 \%$ & $0.63[0.11,3.65]$ \\
\hline EORTC-GELA H9-F & $-1.2925(0.9426)$ & + & $4.9 \%$ & $0.27[0.04,1.74]$ \\
\hline GATLA 9-H-77 & $-0.3484(0.365 I)$ & $=$ & $31.0 \%$ & $0.71[0.35,1.44]$ \\
\hline Subtotal $(95 \% \mathrm{CI})$ & & - & $41.3 \%$ & $0.62[0.33,1.17]$ \\
\hline \multicolumn{5}{|c|}{ Heterogeneity: $\mathrm{Tau}^{2}=0.0 ; \mathrm{Chi}^{2}=0.87, \mathrm{df}=2(\mathrm{P}=0.65) ; \mathrm{I}^{2}=0.0 \%$} \\
\hline \multicolumn{5}{|c|}{ Test for overall effect: $Z=1.48(P=0.14)$} \\
\hline \multicolumn{5}{|c|}{2 Extended field } \\
\hline Mexico B2H03I & $-1.225(0.2852)$ & + & $48.9 \%$ & $0.29[0.17,0.51]$ \\
\hline Subtotal $(95 \% \mathrm{CI})$ & & - & $48.9 \%$ & $0.29[0.17,0.51]$ \\
\hline \multicolumn{5}{|c|}{ Heterogeneity: not applicable } \\
\hline \multicolumn{5}{|c|}{ Test for overall effect: $Z=4.30(P=0.000017)$} \\
\hline \multicolumn{5}{|c|}{3 Mixed } \\
\hline MSKCC trial \#90-44 & $-1.167 \mid(0.6667)$ & $\longrightarrow$ & $9.7 \%$ & $0.31[0.08,1.15]$ \\
\hline Subtotal $(95 \% \mathrm{CI})$ & & - & $9.7 \%$ & $0.31[0.08,1.15]$ \\
\hline \multicolumn{5}{|c|}{ Heterogeneity: not applicable } \\
\hline \multicolumn{5}{|c|}{ Test for overall effect: $Z=1.75(P=0.080)$} \\
\hline Total $(95 \% \mathrm{CI})$ & & $\bullet$ & $100.0 \%$ & $0.40[0.27,0.61]$ \\
\hline \multicolumn{5}{|c|}{ Heterogeneity: $\operatorname{Tau}^{2}=0.01 ; \mathrm{Chi}^{2}=4.14, \mathrm{df}=4(\mathrm{P}=0.39) ; \mathrm{I}^{2}=3 \%$} \\
\hline Test for overall effect: $Z=$ & $0.000015)$ & & & \\
\hline
\end{tabular}




\section{Analysis I.7. Comparison I Overall Survival, Outcome 7 Type of chemotherapy.}

Review: Chemotherapy alone versus chemotherapy plus radiotherapy for early stage Hodgkin lymphoma

Comparison: I Overall Survival

Outcome: 7 Type of chemotherapy

Study or subgroup $\quad \log [$ Hazard Ratio $]$

(SE)

$\begin{array}{ll}\text { I ABVD } & \\ \text { Mexico B2H03। } & -1.2245(0.2852) \\ \text { MSKCC trial \#90-44 } & -1.1733(0.6667)\end{array}$

Subtotal (95\% CI)

Heterogeneity: $\mathrm{Tau}^{2}=0.0 ; \mathrm{Chi}^{2}=0.00, \mathrm{df}=\mathrm{I}(\mathrm{P}=0.94) ; \mathrm{I}^{2}=0.0 \%$

Test for overall effect: $Z=4.64(P<0.0000 I)$

2 CVPP
CALGB 775।
$-0.47(0.9)$
GATLA 9-H-77
$-0.3786(0.3651)$

Subtotal (95\% CI)

Heterogeneity: $\mathrm{Tau}^{2}=0.0 ; \mathrm{Chi}^{2}=0.0 \mathrm{I}, \mathrm{df}=\mathrm{I}(\mathrm{P}=0.93) ; \mathrm{I}^{2}=0.0 \%$

Test for overall effect: $Z=1.16(P=0.25)$

3 EBVP

EORTC-GELA H9-F

$-1.2931(0.9407)$

Subtotal (95\% CI)

Heterogeneity: not applicable

Test for overall effect: $Z=1.37(P=0.17)$

Total (95\% CI)

Heterogeneity: $\mathrm{Tau}^{2}=0.0 ; \mathrm{Chi}^{2}=3.89, \mathrm{df}=4(\mathrm{P}=0.42) ; \mathrm{I}^{2}=0.0 \%$

Test for overall effect: $Z=4.57(P<0.0000$ I $)$
Hazard Ratio

Weight

Hazard Ratio IV,Random,95\% Cl

$0.31[0.08,1.14]$

$59.6 \%$

$0.30[0.18,0.50]$

$5.1 \%$

$30.7 \%$

$0.63[0.11,3.65]$

$0.68[0.33,1.40]$

$35.8 \%$

$0.68[0.35,1.31]$

$4.6 \%$

$0.27[0.04,1.73]$

$4.6 \%$

$0.27[0.04,1.73]$

$100.0 \%$

$0.40[0.27,0.59]$

$\begin{array}{lllll}0.01 & 0.1 & 1 & 10 & 100\end{array}$




\section{Analysis I.8. Comparison I Overall Survival, Outcome 8 Length of follow-up.}

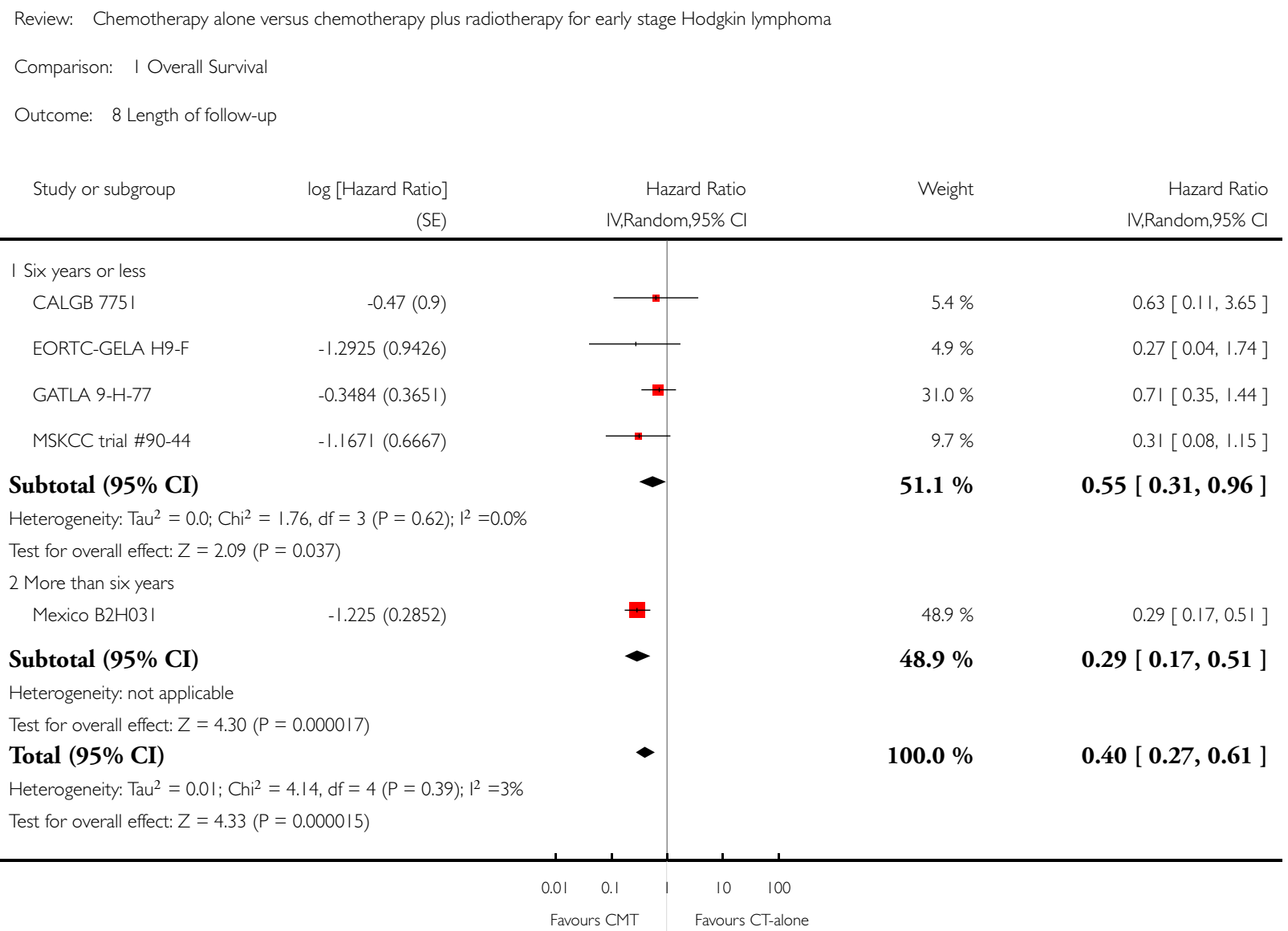




\section{Analysis I.9. Comparison I Overall Survival, Outcome 94 year survival in the CT group.}

Review: Chemotherapy alone versus chemotherapy plus radiotherapy for early stage Hodgkin lymphoma

Comparison: I Overall Survival

Outcome: 94 year survival in the CT group

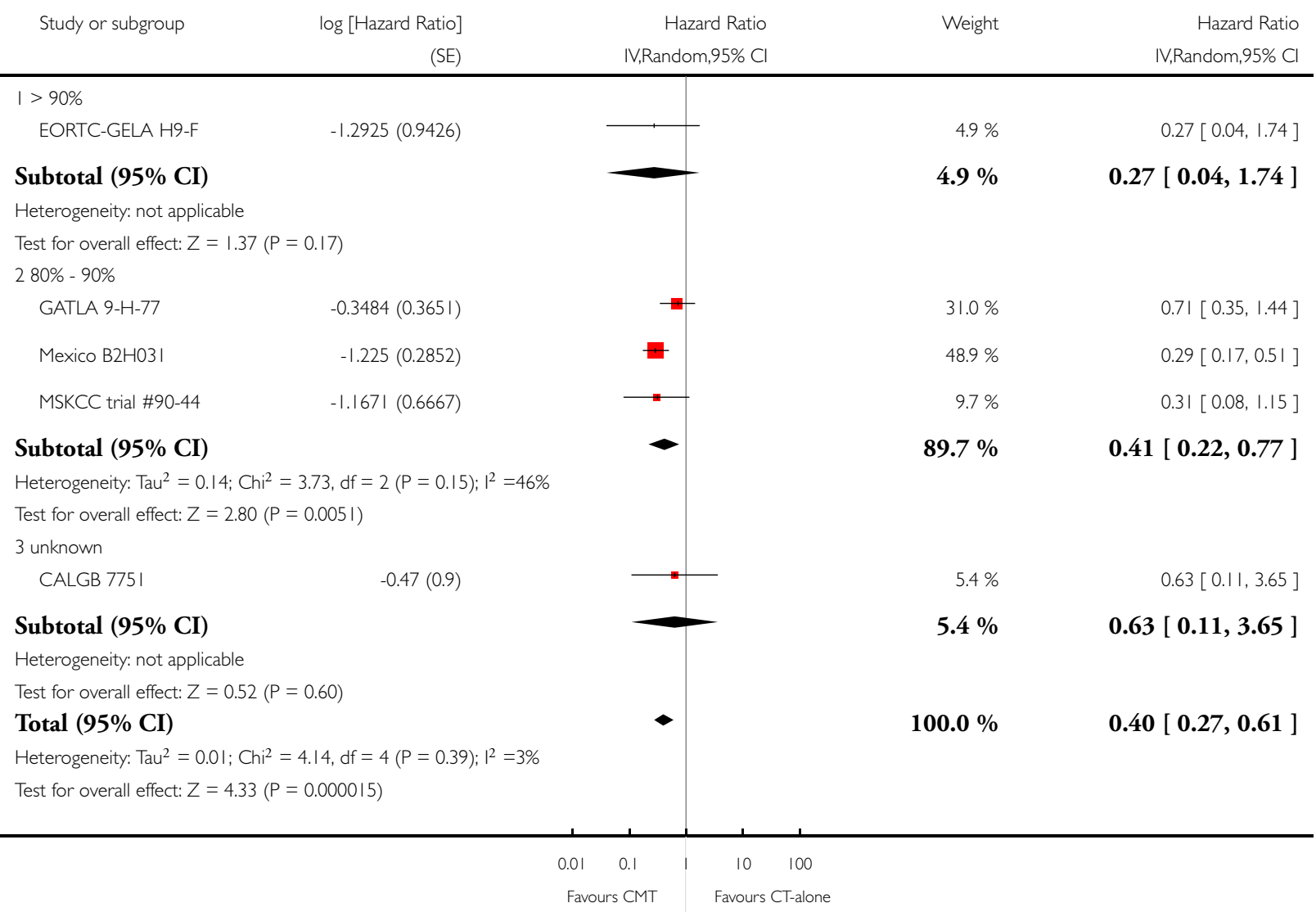




\section{Analysis I.I0. Comparison I Overall Survival, Outcome I0 Allocation concealment.}

Review: Chemotherapy alone versus chemotherapy plus radiotherapy for early stage Hodgkin lymphoma

Comparison: I Overall Survival

Outcome: 10 Allocation concealment

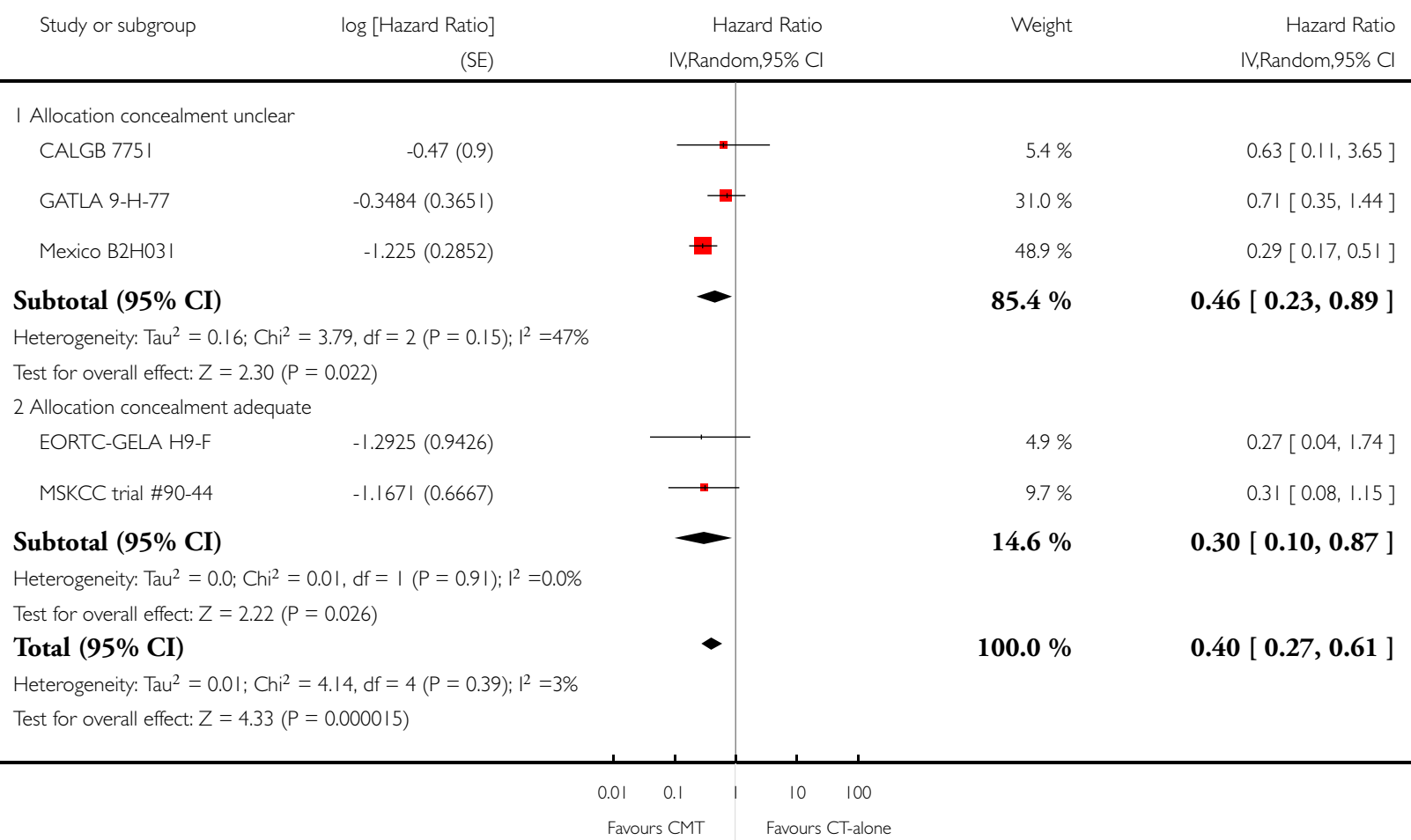




\section{Analysis I.II. Comparison I Overall Survival, Outcome I I ITT-analysis.}

Review: Chemotherapy alone versus chemotherapy plus radiotherapy for early stage Hodgkin lymphoma

Comparison: I Overall Survival

Outcome: II ITT-analysis

Study or subgroup $\quad \log$ [Hazard Ratio] Hazard Ratio Height Hazard Ratio

(SE) IV,Random,95\% Cl IV,Random,95\% Cl

I ITT-analysis

EORTC-GELA H9-F

$-1.2925(0.9426)$

$4.9 \%$

$0.27[0.04,1.74]$

MSKCC trial \#90-44

$-1.167 \mid(0.6667)$

$9.7 \%$

$0.31[0.08,1.15]$

Subtotal (95\% CI)

$14.6 \%$

$0.30[0.10,0.87]$

Heterogeneity: $\mathrm{Tau}^{2}=0.0 ; \mathrm{Chi}^{2}=0.0 \mathrm{I}, \mathrm{df}=\mathrm{I}(\mathrm{P}=0.9 \mathrm{I}) ; \mathrm{I}^{2}=0.0 \%$

Test for overall effect: $Z=2.22(P=0.026)$

2 No ITT-analysis

CALGB 775 I

$-0.47(0.9)$

GATLA 9-H-77

$-0.3484(0.365 I)$

Mexico $\mathrm{B} 2 \mathrm{HO} 3$

$-1.225(0.2852)$

Subtotal $(\mathbf{9 5 \%} \mathrm{CI})$

Heterogeneity: $\operatorname{Tau}^{2}=0.16 ; \mathrm{Chi}^{2}=3.79, \mathrm{df}=2(\mathrm{P}=0.15) ; \mathrm{I}^{2}=47 \%$

Test for overall effect: $Z=2.30(P=0.022)$

Total (95\% CI)

Heterogeneity: $\mathrm{Tau}^{2}=0.0 \mathrm{I} ; \mathrm{Chi}^{2}=4.14, \mathrm{df}=4(\mathrm{P}=0.39) ; \mathrm{I}^{2}=3 \%$

Test for overall effect: $Z=4.33(P=0.000015)$
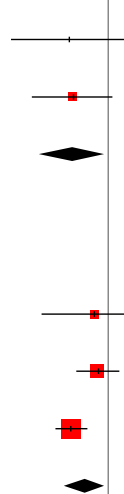

$\longrightarrow$

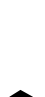

$\bullet$
$0.63[0.11,3.65]$

$0.7 \mid[0.35,1.44]$

$0.29[0.17,0.51]$

$0.46[0.23,0.89]$
$85.4 \%$

$100.0 \%$ 


\section{Analysis 2.I. Comparison 2 Progression-Free Survival, Outcome I All trials.}

Review: Chemotherapy alone versus chemotherapy plus radiotherapy for early stage Hodgkin lymphoma

Comparison: 2 Progression-Free Survival

Outcome: I All trials

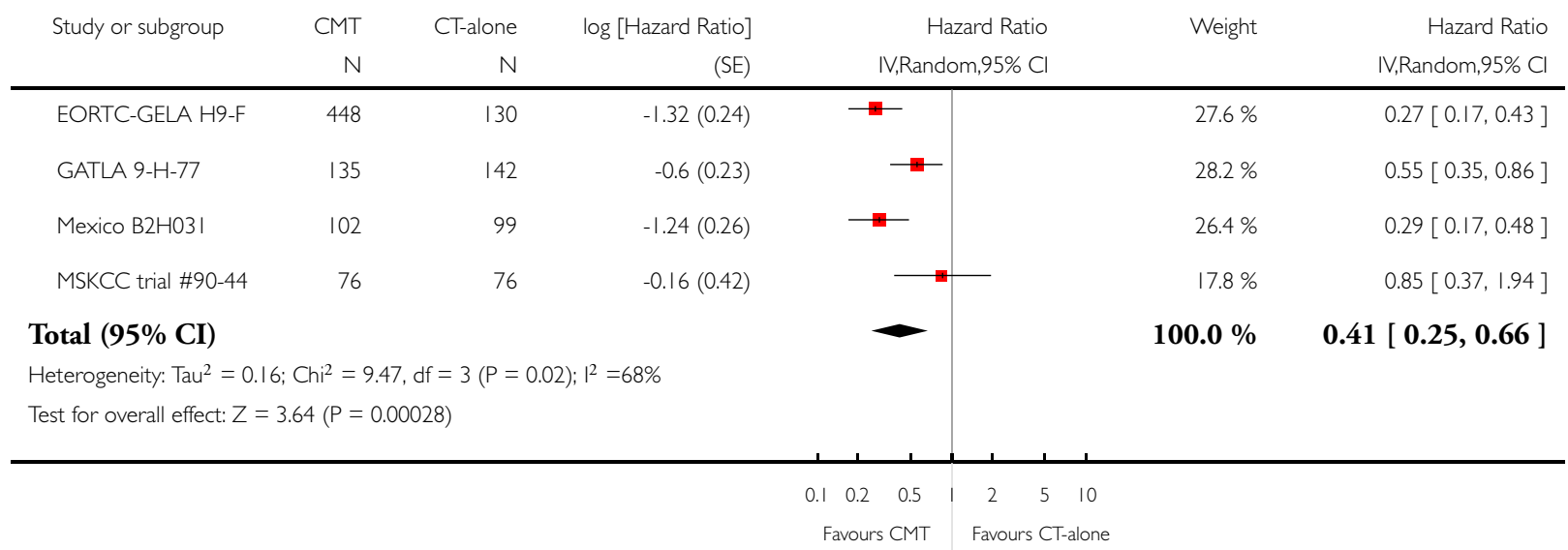

\section{Analysis 2.2. Comparison 2 Progression-Free Survival, Outcome 2 Type of chemotherapy.}

Review: Chemotherapy alone versus chemotherapy plus radiotherapy for early stage Hodgkin lymphoma

Comparison: 2 Progression-Free Survival

Outcome: 2 Type of chemotherapy

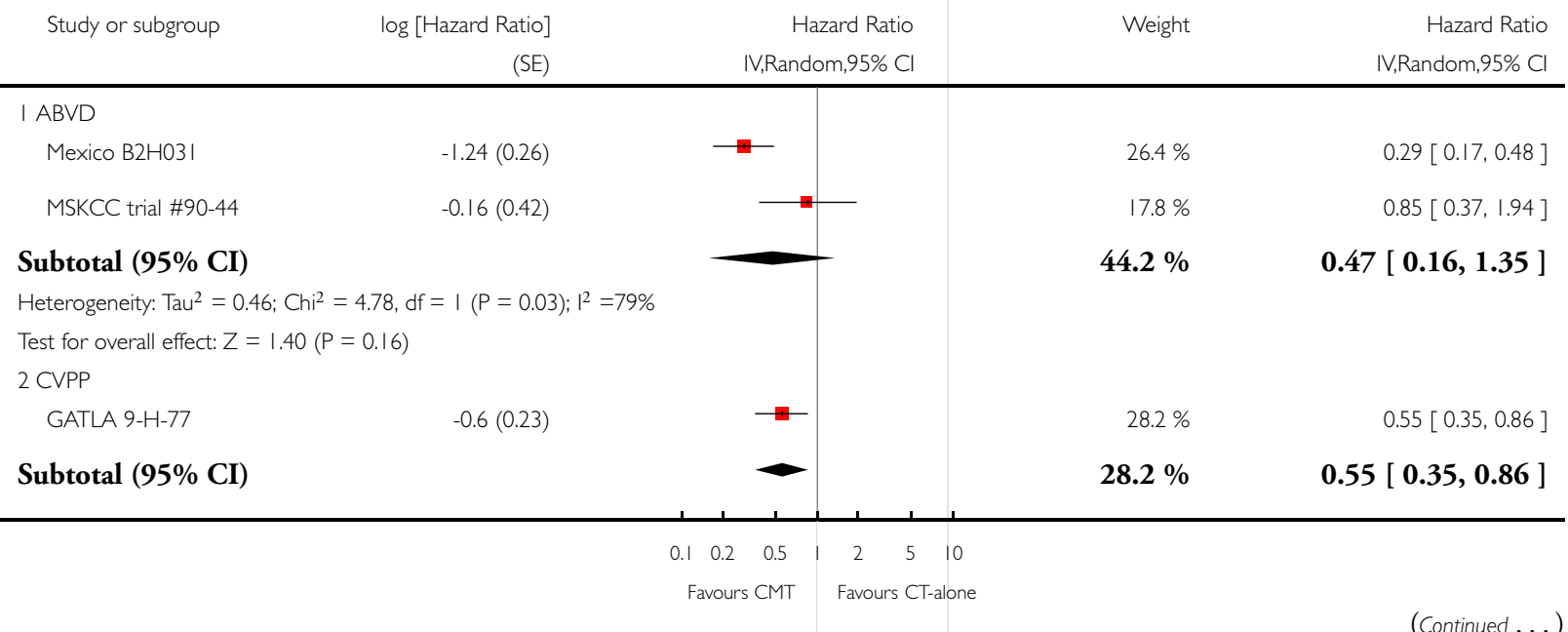




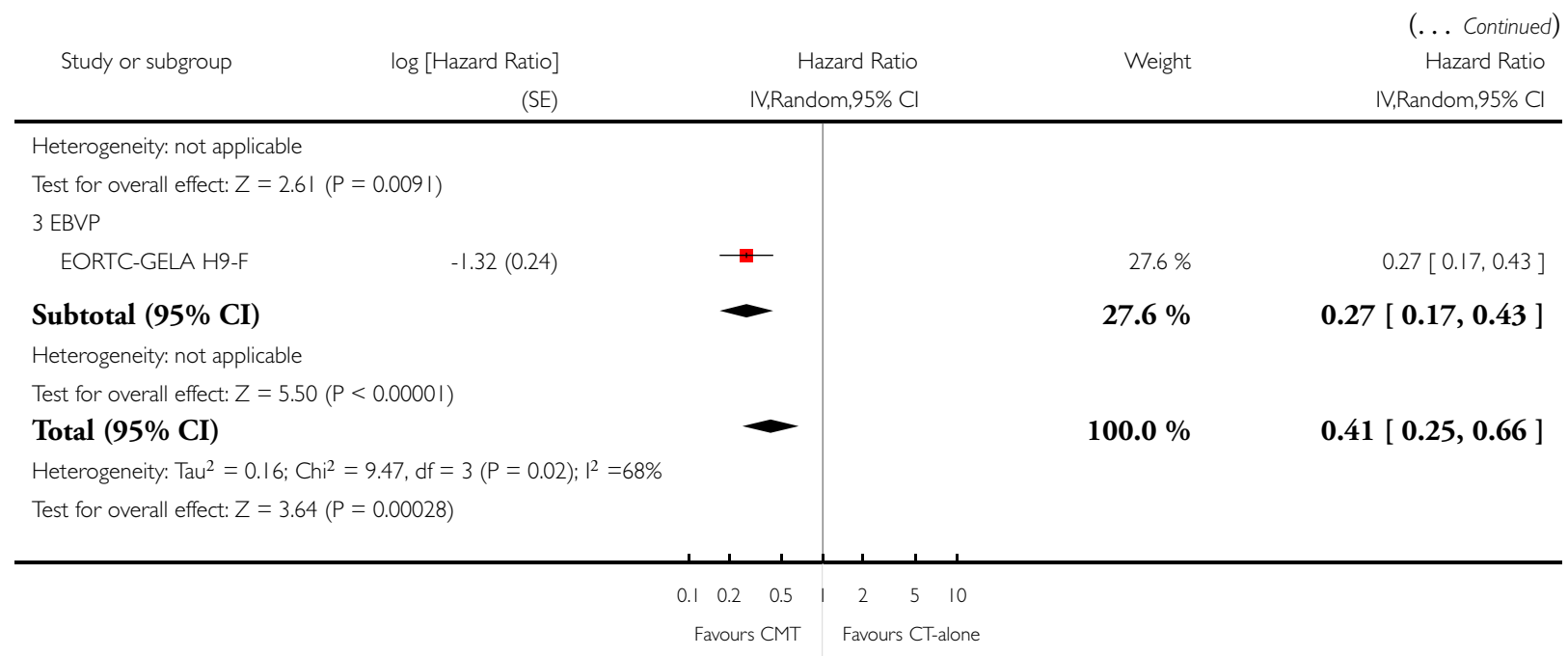

\section{Analysis 2.3. Comparison 2 Progression-Free Survival, Outcome 3 Proportion of patients early favourable.}

Review: Chemotherapy alone versus chemotherapy plus radiotherapy for early stage Hodgkin lymphoma

Comparison: 2 Progression-Free Survival

Outcome: 3 Proportion of patients early favourable

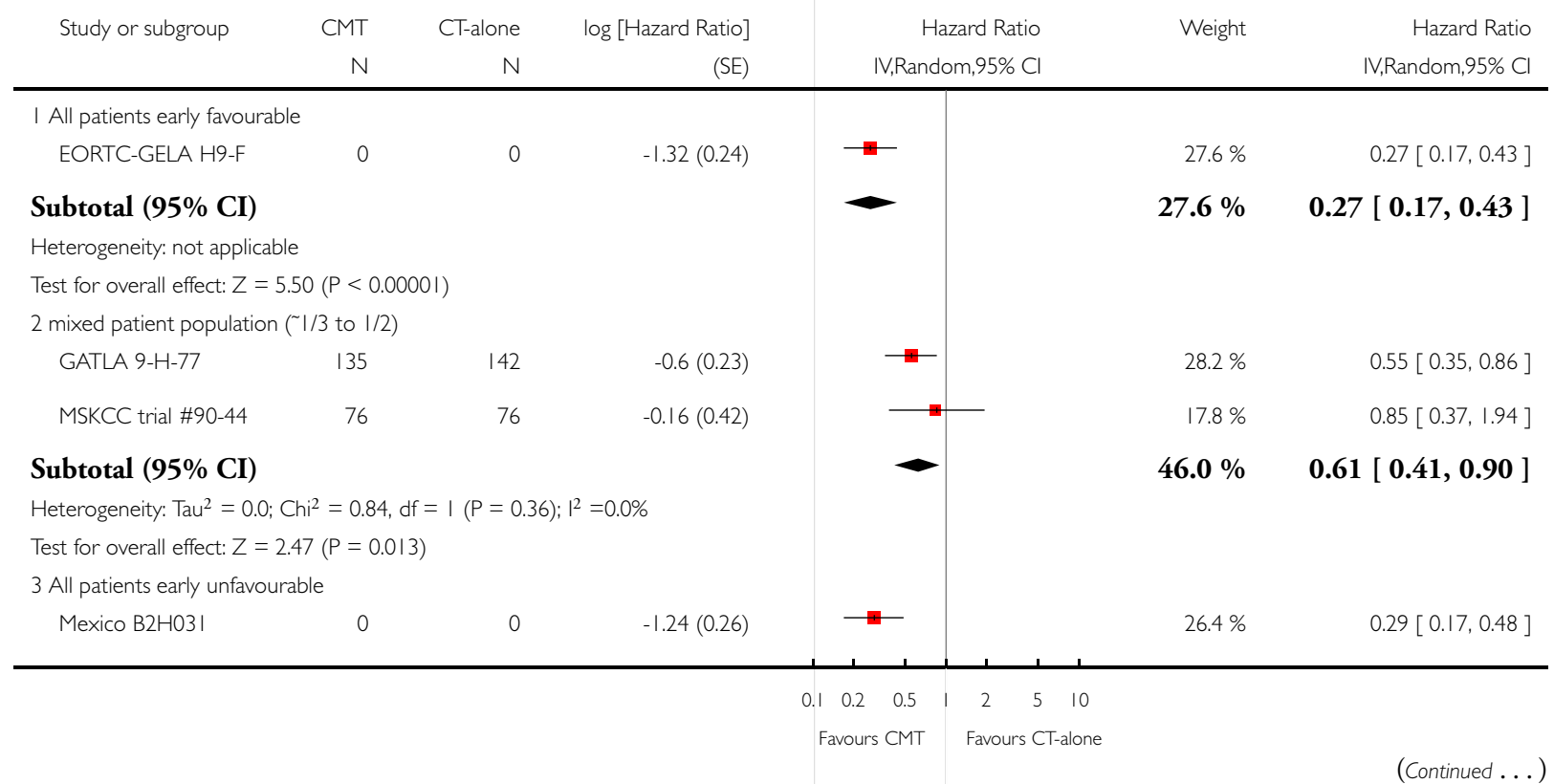




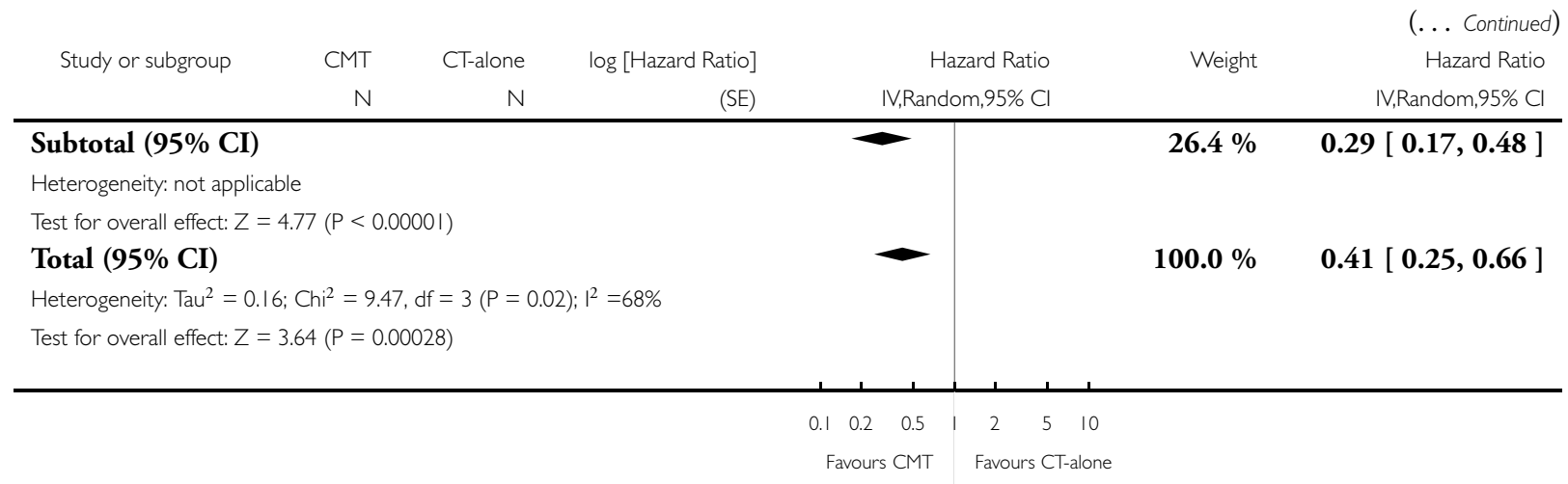

\section{Analysis 2.4. Comparison 2 Progression-Free Survival, Outcome 4 Definition of progression.}

Review: Chemotherapy alone versus chemotherapy plus radiotherapy for early stage Hodgkin lymphoma

Comparison: 2 Progression-Free Survival

Outcome: 4 Definition of progression

Study or subgroup $\quad \log$ [Hazard Ratio] Hazard Ratio Hazht Ratio IV,Random,95\% Cl IV,Random,95\% Cl

I In CR patients
EORTC-GELA H9-F
Subtotal $(\mathbf{9 5 \%} \mathbf{C I})$
Heterogeneity: not applicable
Test for overall effect: Z = $5.50(\mathrm{P}<0.0000$ । $)$
2 Progress in all patients
$\quad$ GATLA 9-H-77
MSKCC trial \#90-44

Subtotal (95\% CI)

Heterogeneity: $\mathrm{Tau}^{2}=0.0 ; \mathrm{Chi}^{2}=0.84, \mathrm{df}=\mathrm{I}(\mathrm{P}=0.36) ; \mathrm{I}^{2}=0.0 \%$

Test for overall effect: $Z=2.47(P=0.013)$ 3 Unclear
Mexico $\mathrm{B} 2 \mathrm{HO} 3$ I
$-1.24(0.26)$

Subtotal (95\% CI)

Heterogeneity: not applicable

Test for overall effect: $Z=4.77(P<0.0000$ I)

Total (95\% CI)

Heterogeneity: $\mathrm{Tau}^{2}=0.16 ; \mathrm{Chi}^{2}=9.47, \mathrm{df}=3(\mathrm{P}=0.02) ; \mathrm{I}^{2}=68 \%$

Test for overall effect: $Z=3.64(P=0.00028)$ 


\section{Analysis 2.5. Comparison 2 Progression-Free Survival, Outcome 5 Timing of radiotherapy.}

Review: Chemotherapy alone versus chemotherapy plus radiotherapy for early stage Hodgkin lymphoma

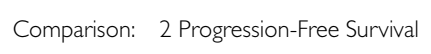

I Radiotherapy after chemotherapy

EORTC-GELA H9-F

MSKCC trial \#90-44

Subtotal (95\% CI)

Heterogeneity: Tau $^{2}=0.56 ; \mathrm{Chi}^{2}=5.75, \mathrm{df}=\mathrm{I}(\mathrm{P}=0.02) ; \mathrm{I}^{2}=83 \%$

Test for overall effect: $Z=1.37(P=0.17)$

2 Sandwich technique (CT-RT-CT)

$\begin{array}{lr}\text { GATLA 9-H-77 } & -0.6(0.23) \\ \text { Mexico B2H03I } & -1.24(0.26)\end{array}$

Subtotal $(95 \%$ CI $)$

Heterogeneity: Tau $^{2}=0.14 ; \mathrm{Chi}^{2}=3.40, \mathrm{df}=1(\mathrm{P}=0.07) ; \mathrm{I}^{2}=71 \%$ Test for overall effect: $Z=2.84(P=0.0045)$

Total (95\% CI)

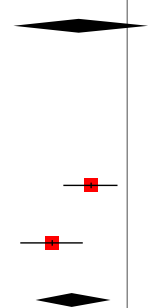

Heterogeneity: $\mathrm{Tau}^{2}=0.16 ; \mathrm{Chi}^{2}=9.47, \mathrm{df}=3(\mathrm{P}=0.02) ; \mathrm{I}^{2}=68 \%$ Test for overall effect: $Z=3.64(P=0.00028)$

$\begin{array}{ll}28.2 \% & 0.55[0.35,0.86] \\ 26.4 \% & 0.29[0.17,0.48]\end{array}$

$54.6 \%$

$0.40[0.22,0.75]$

$100.0 \%$

$0.41[0.25,0.66]$ 


\section{Analysis 2.6. Comparison 2 Progression-Free Survival, Outcome 6 Type of radiotherapy.}

Review: Chemotherapy alone versus chemotherapy plus radiotherapy for early stage Hodgkin lymphoma

Comparison: 2 Progression-Free Survival

Outcome: 6 Type of radiotherapy

Study or subgroup $\quad \log [$ Hazard Ratio

$(\mathrm{SE})$

\begin{tabular}{ll}
\hline I Involved field & \\
EORTC-GELA H9-F & $-1.32(0.24)$ \\
GATLA 9-H-77 & $-0.6(0.23)$
\end{tabular}

Subtotal (95\% CI)

Heterogeneity: $\mathrm{Tau}^{2}=0.20 ; \mathrm{Chi}^{2}=4.69, \mathrm{df}=\mathrm{I}(\mathrm{P}=0.03) ; \mathrm{I}^{2}=79 \%$

Test for overall effect: $Z=2.66(P=0.0079)$

2 Extended field

$$
\text { Mexico } \mathrm{B} 2 \mathrm{HO} 3 \mathrm{I}
$$

\section{Subtotal (95\% CI)}

Heterogeneity: not applicable

Test for overall effect: $Z=4.77(P<0.0000$ I $)$

3 Mixed
MSKCC trial \#90-44
$-0.16(0.42)$

Subtotal (95\% CI)

Heterogeneity: not applicable

Test for overall effect: $Z=0.38(P=0.70)$

Total (95\% CI)

Heterogeneity: $\mathrm{Tau}^{2}=0.16 ; \mathrm{Chi}^{2}=9.47, \mathrm{df}=3(\mathrm{P}=0.02) ; \mathrm{I}^{2}=68 \%$ Test for overall effect: $Z=3.64(P=0.00028)$
Hazard Ratio IV,Random,95\% Cl

Weight

Hazard Ratio IV,Random,95\% Cl

$\rightarrow-$

$26.4 \%$

$0.29[0.17,0.48]$

$26.4 \%$

$0.29[0.17,0.48$ ]

$17.8 \%$

$0.85[0.37,1.94]$

$17.8 \%$

$0.85[0.37,1.94$ ]

$100.0 \%$

0.41 [ $0.25,0.66$ ] 


\section{Analysis 2.7. Comparison 2 Progression-Free Survival, Outcome 7 Length of follow-up.}

Review: Chemotherapy alone versus chemotherapy plus radiotherapy for early stage Hodgkin lymphoma

Comparison: 2 Progression-Free Survival

Outcome: 7 Length of follow-up

Study or subgroup $\quad \log [$ Hazard Ratio

(SE)

\begin{tabular}{lr}
\hline I Median follow-up $\leq 6$ years & \\
EORTC-GELA H9-F & $-1.32(0.24)$ \\
GATLA 9-H-77 & $-0.6(0.23)$ \\
MSKCC trial \#90-44 & $-0.16(0.42)$
\end{tabular}

\section{Subtotal (95\% CI)}

Heterogeneity: Tau $^{2}=0.22 ; \mathrm{Chi}^{2}=7.7 \mathrm{l}, \mathrm{df}=2(\mathrm{P}=0.02) ; \mathrm{I}^{2}=74 \%$

Test for overall effect: $Z=2.33(P=0.020)$

2 Median follow-up $>6$ years
Mexico $\mathrm{B} 2 \mathrm{HO} 3$
$-1.24(0.26)$

Subtotal (95\% CI)

Heterogeneity: not applicable

Test for overall effect: $Z=4.77(P<0.0000$ I)

Total (95\% CI)

Heterogeneity: $\mathrm{Tau}^{2}=0.16 ; \mathrm{Chi}^{2}=9.47, \mathrm{df}=3(\mathrm{P}=0.02) ; \mathrm{I}^{2}=68 \%$ Test for overall effect: $Z=3.64(P=0.00028)$
Hazard Ratio

Weight

Hazard Ratio IV,Random,95\% Cl

$27.6 \%$

$28.2 \%$

$17.8 \%$

$73.6 \%$

\section{$\begin{array}{lllllll}0.1 & 0.2 & 0.5 & 1 & 2 & 5 & 10\end{array}$}

Favours CMT Favours CT-alone 


\section{Analysis 2.8. Comparison 2 Progression-Free Survival, Outcome 8 Allocation concealment.}

Review: Chemotherapy alone versus chemotherapy plus radiotherapy for early stage Hodgkin lymphoma

Comparison: 2 Progression-Free Survival

Outcome: 8 Allocation concealment

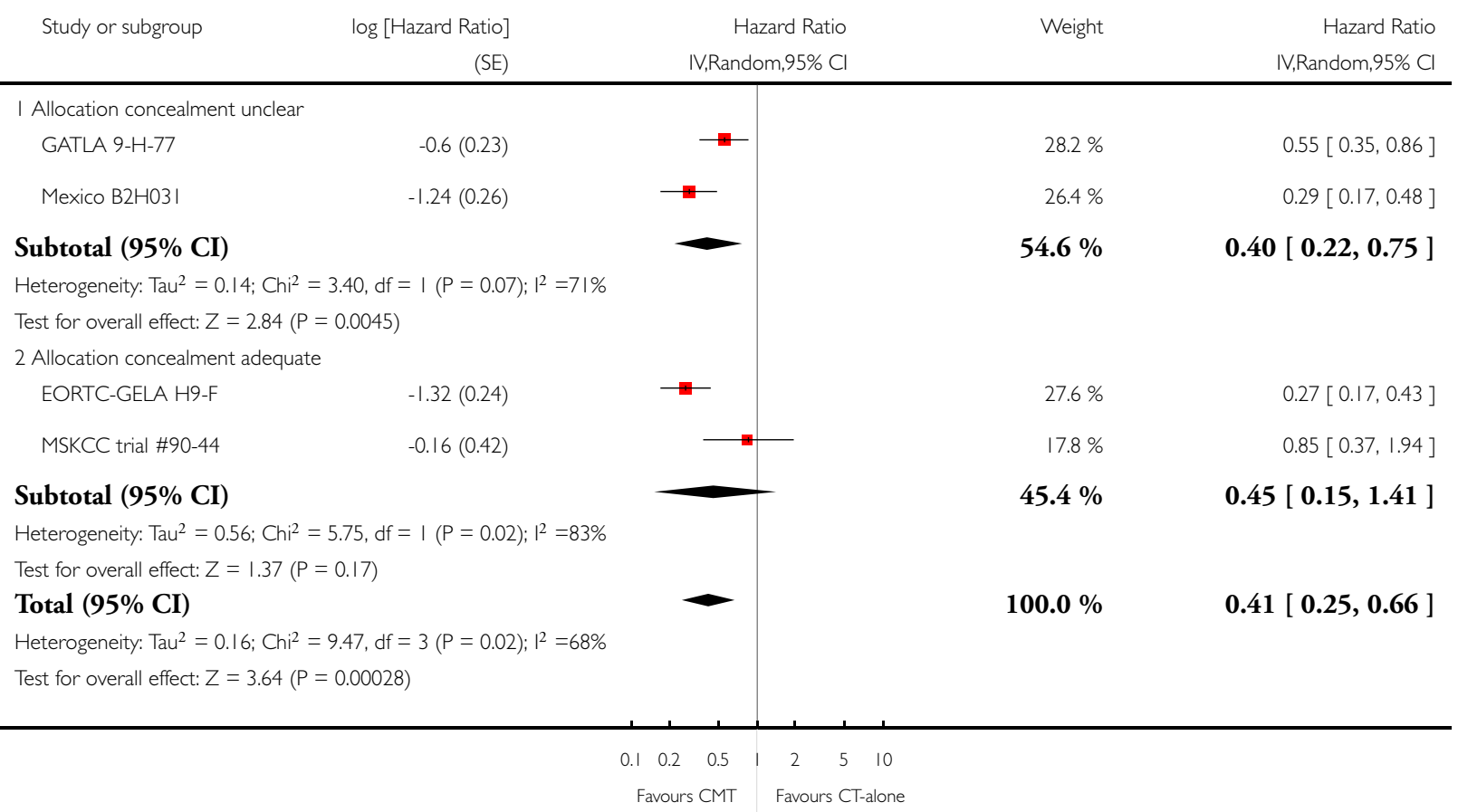




\section{Analysis 2.9. Comparison 2 Progression-Free Survival, Outcome 9 ITT-Analysis.}

Review: Chemotherapy alone versus chemotherapy plus radiotherapy for early stage Hodgkin lymphoma

Comparison: 2 Progression-Free Survival

Outcome: 9 ITT-Analysis

Study or subgroup $\quad \log$ [Hazard Ratio] Hazard Ratio Height Hazard Ratio

(SE) IV,Random,95\% Cl IV,Random,95\% Cl

I ITT-analysis

EORTC-GELA H9-F

$-1.32(0.24)$

$\longrightarrow$

$27.7 \%$

$0.27[0.17,0.43]$

MSKCC trial \#90-44

$-0.21(0.42)$

$17.6 \%$

$0.81[0.36,1.85]$

Subtotal (95\% CI)

$45.3 \%$

$0.44[0.15,1.30]$

Heterogeneity: Tau $^{2}=0.50 ; \mathrm{Chi}^{2}=5.27, \mathrm{df}=\mathrm{I}(\mathrm{P}=0.02) ; \mathrm{I}^{2}=81 \%$

Test for overall effect: $Z=1.48(P=0.14)$

2 No ITT-analysis

GATLA 9-H-77

$-0.6(0.23)$

$28.3 \%$

$0.55[0.35,0.86]$

Mexico $\mathrm{B} 2 \mathrm{HO} 3$

$-1.24(0.26)$

$26.4 \%$

$0.29[0.17,0.48]$

Subtotal $(95 \%$ CI $)$

$54.7 \%$

$0.40[0.22,0.75]$

Heterogeneity: Tau $^{2}=0.14 ; \mathrm{Chi}^{2}=3.40, \mathrm{df}=|(P=0.07) ;|^{2}=71 \%$

Test for overall effect: $Z=2.84(P=0.0045)$

Total (95\% CI)

$100.0 \%$

$0.41[0.25,0.65]$

Heterogeneity: Tau $^{2}=0.15 ; \mathrm{Chi}^{2}=9.04, \mathrm{df}=3(\mathrm{P}=0.03) ; \mathrm{I}^{2}=67 \%$

Test for overall effect: $Z=3.77(P=0.00017)$

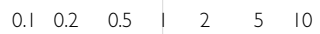

Favours CMT Favours CT-alone 


\section{Analysis 3.I. Comparison 3 Complete Response Rate, Outcome I All trials.}

Review: Chemotherapy alone versus chemotherapy plus radiotherapy for early stage Hodgkin lymphoma

Comparison: 3 Complete Response Rate

Outcome: I All trials

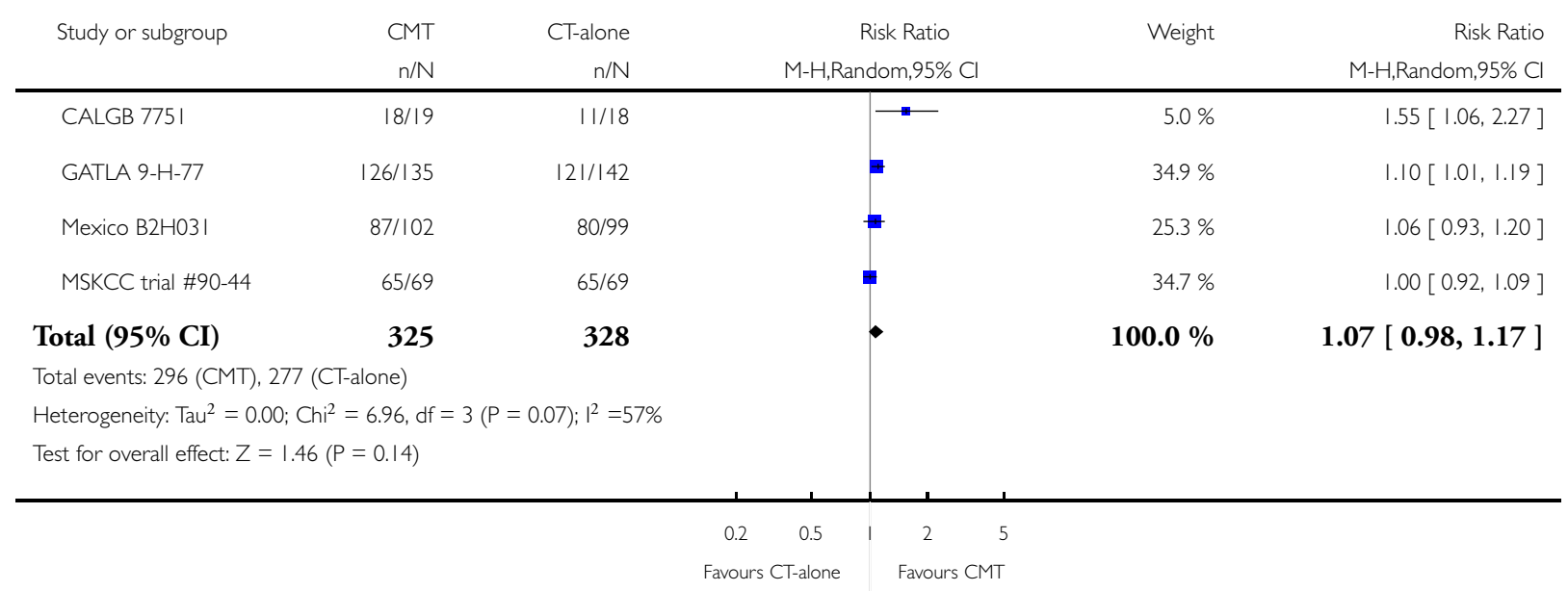




\section{Analysis 3.2. Comparison 3 Complete Response Rate, Outcome 2 Type of Chemotherapy.}

Review: Chemotherapy alone versus chemotherapy plus radiotherapy for early stage Hodgkin lymphoma

Comparison: 3 Complete Response Rate

Outcome: 2 Type of Chemotherapy

Study or subgroup

CT-alone

$\mathrm{n} / \mathrm{N} \quad \mathrm{n} / \mathrm{N} \quad \mathrm{M}-\mathrm{H}$, Random,95\% Cl

Risk Ratio

Weight

Risk Ratio $\mathrm{M}-\mathrm{H}, \mathrm{Random}, 95 \% \mathrm{Cl}$

I CVPP
CALGB 775।
GATLA 9-H-77

Subtotal (95\% CI)

$18 / 19 \quad 11 / 18$

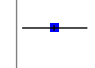

$21 / 142$

154

160

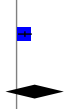

$5.0 \%$

$1.55[1.06,2.27]$

Total events: 144 (CMT), 132 (CT-alone)

Heterogeneity: Tau $^{2}=0.05 ; \mathrm{Chi}^{2}=3.26, \mathrm{df}=\mathrm{I}(\mathrm{P}=0.07) ; \mathrm{I}^{2}=69 \%$

Test for overall effect: $Z=1.25(P=0.21)$

2 ABVD

$\begin{array}{lrr}\text { Mexico B2H03I } & 87 / 102 & 80 / 99 \\ \text { MSKCC trial \#90-44 } & 65 / 69 & 65 / 69\end{array}$

171

168

Subtotal (95\% CI)

Total events: I52 (CMT), I 45 (CT-alone)

Heterogeneity: $\operatorname{Tau}^{2}=0.0 ; \mathrm{Chi}^{2}=0.65, \mathrm{df}=\mathrm{I}(\mathrm{P}=0.42) ; \mathrm{I}^{2}=0.0 \%$

Test for overall effect: $Z=0.47(P=0.64)$

Total (95\% CI)

Total events: 296 (CMT), 277 (CT-alone)

Heterogeneity: $\mathrm{Tau}^{2}=0.00 ; \mathrm{Chi}^{2}=6.96, \mathrm{df}=3(\mathrm{P}=0.07) ; \mathrm{I}^{2}=57 \%$

Test for overall effect: $Z=1.46(P=0.14)$

$\begin{array}{ccccc}0.2 & 0.5 & 2 & 5 \\ \text { Favours } & \text { CT-alone } & & \text { Favours CMT }\end{array}$




\section{Analysis 3.3. Comparison 3 Complete Response Rate, Outcome 3 ITT-analysis.}

Review: Chemotherapy alone versus chemotherapy plus radiotherapy for early stage Hodgkin lymphoma

Comparison: 3 Complete Response Rate

Outcome: 3 ITT-analysis

$\begin{array}{lllll}\text { Study or subgroup } & \text { CMT } & \text { CT-alone } & \text { Risk Ratio } & \text { Weight }\end{array}$

$\mathrm{n} / \mathrm{N} \quad \mathrm{n} / \mathrm{N} \quad \mathrm{M}-\mathrm{H}$, Random,95\% Cl M-H,Random,95\% Cl

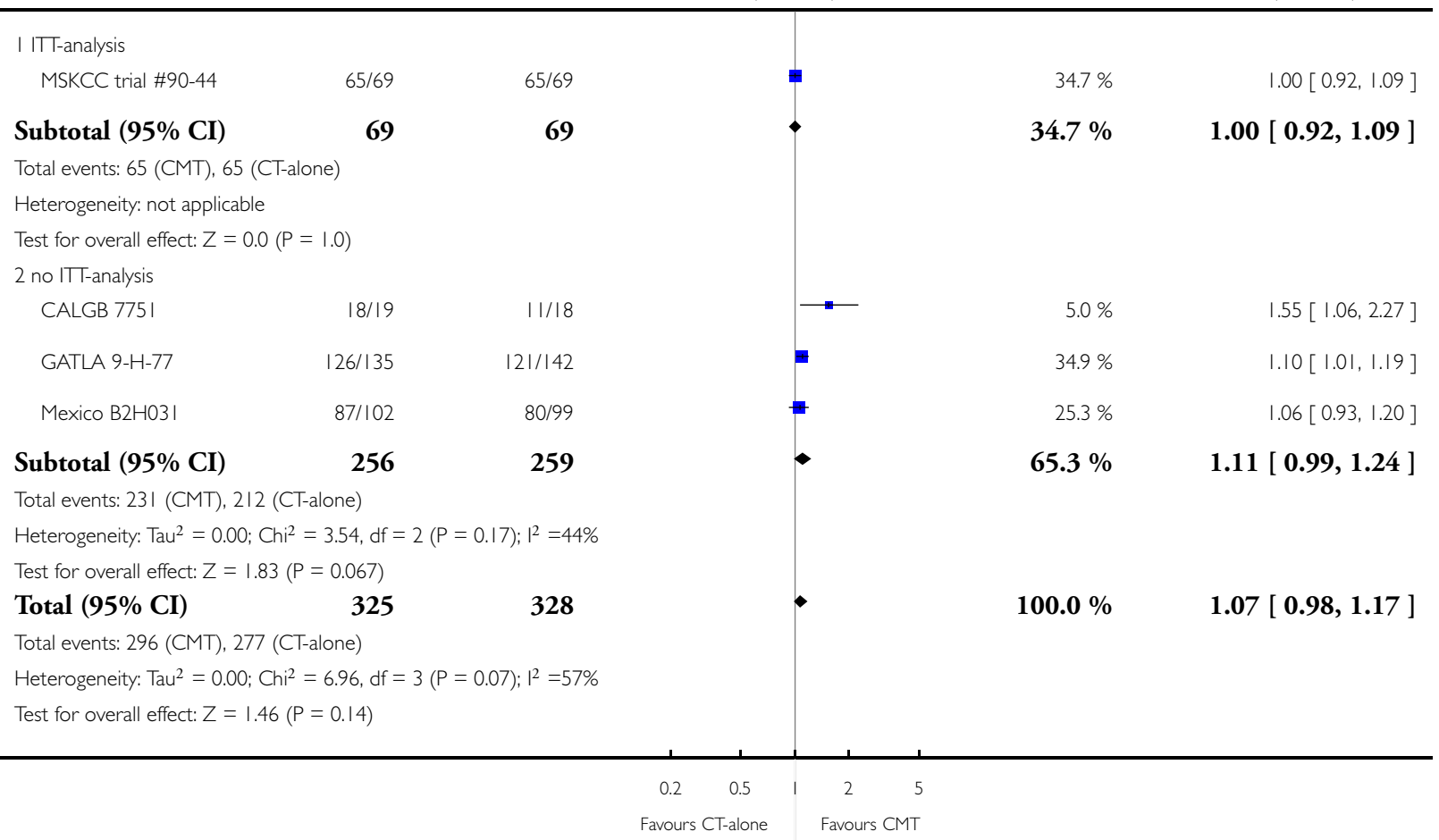




\section{Analysis 3.4. Comparison 3 Complete Response Rate, Outcome 4 Number of evaluable patients.}

Review: Chemotherapy alone versus chemotherapy plus radiotherapy for early stage Hodgkin lymphoma

Comparison: 3 Complete Response Rate

Outcome: 4 Number of evaluable patients

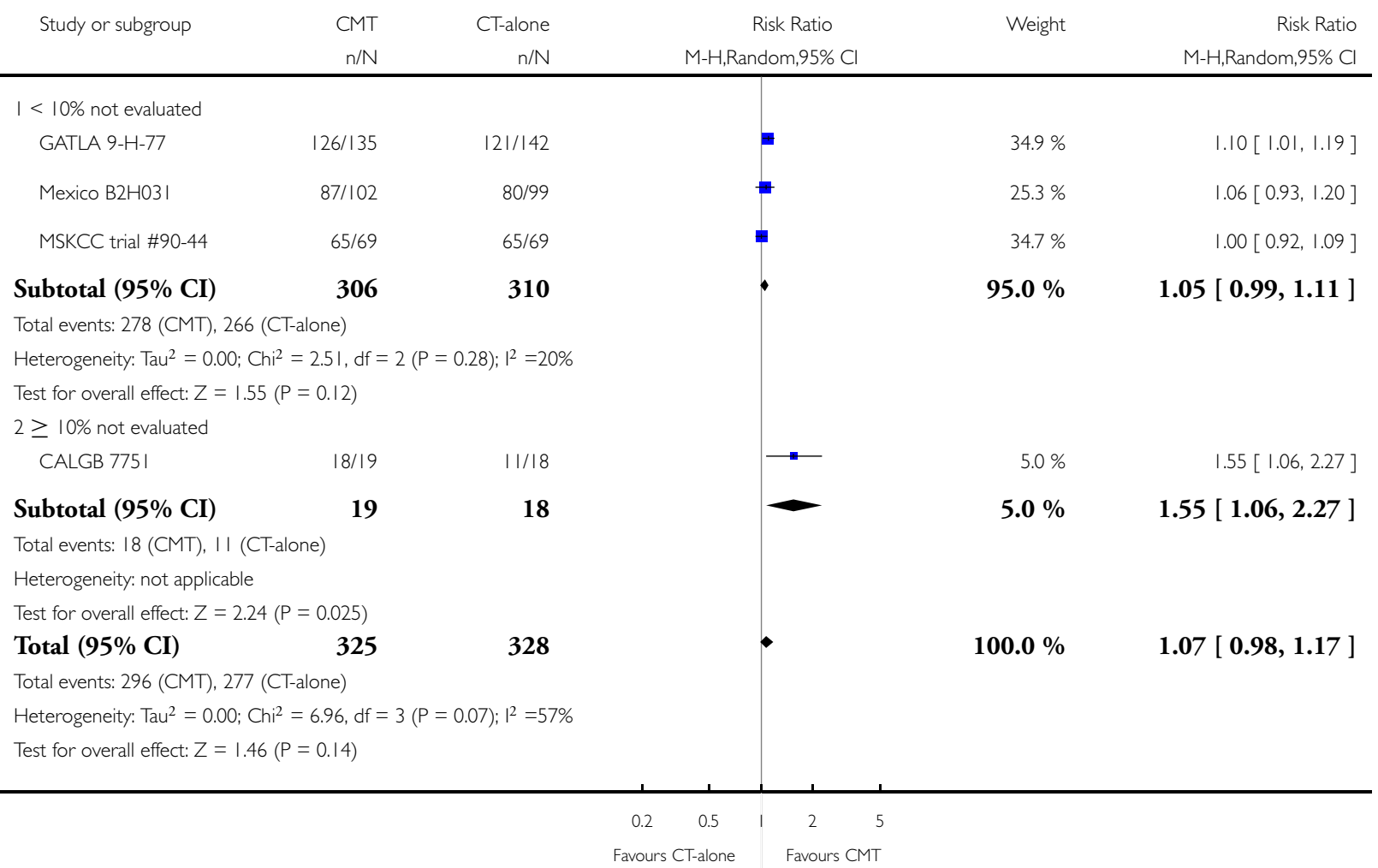


Analysis 4.I. Comparison 4 Overall Response Rate, Outcome I All Trials.

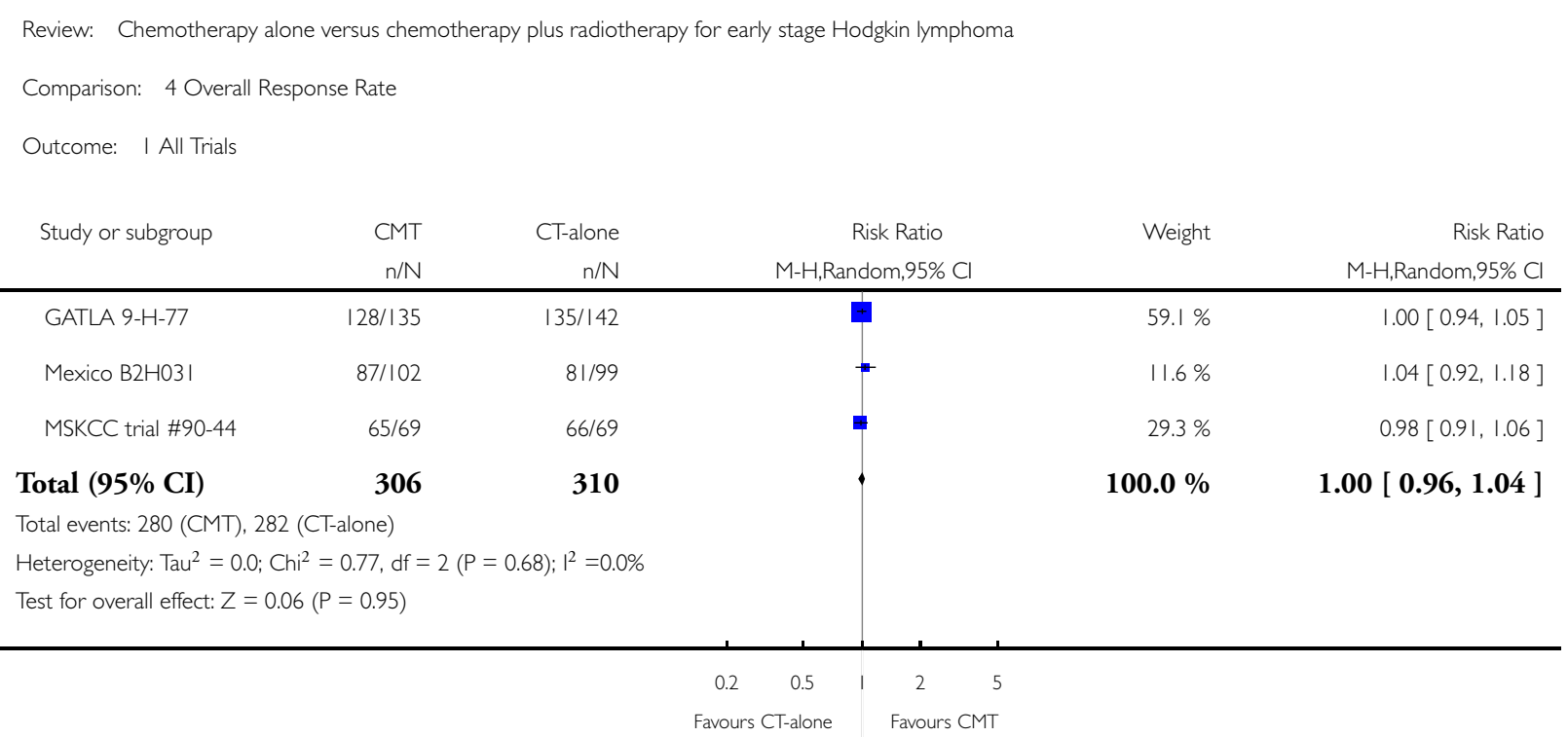

\section{A P PENDICES}

\section{Appendix I. CENTRAL search strategy}

The following search strategy was used to search CENTRAL:

1. (favourable or unfavourable)

2. ((earl* or low* or limit $\left.{ }^{*}\right)$ near/3 $\left(\right.$ stag* $^{*}$ or $\left.\left.\operatorname{grad}^{*}\right)\right)$

3. (intermediate*)

4. (bulky)

5. (\#1 OR \#2 OR \#3 OR \#4)

6. MeSH descriptor LYMPHOMA, this term only

7. MeSH descriptor HODGKIN DISEASE explode all trees

8. (hodgkin* near/2 (disease* or granulom*))

9. (reticulolymphosarcom* or germinoblastom*)

10. (malignan* near/2 (lymphogranulom* or granulom*))

11. (\#6 OR \#7 OR \#8 OR \#9 OR \#10)

12. MeSH descriptor ANTINEOPLASTIC AGENTS explode all trees

13. MeSH descriptor REMISSION INDUCTION explode all trees

14. MeSH descriptor ANTINEOPLASTIC PROTOCOLS explode all trees

15. ((consolidat* or induct* $^{*}$ or maintenance or conditioning*) and (therap* or treat* or regimen* or patient*))

16. ((therap* or induc*) near/3 remission*)

17. (chemotherap* or chemo-therap*)

18. (Antineoplast* or anti-neoplast ${ }^{*}$ )

19. ((cytosta* or cytotox $\left.^{*}\right)$ near/2 (therap* or treat* or regimen $\left.\left.{ }^{*}\right)\right)$

20. MeSH descriptor RADIOTHERAPY explode all trees

21. (radiotherap* or radio-therap*) 
22. (chemoradiotherap* or chemo-radio-therap*)

23. MeSH descriptor COMBINED MODALITY THERAPY explode all trees

24. ((multimodal ${ }^{*}$ or multi-modal $\left.{ }^{*}\right)$ near/3 (treat* $^{*}$ or therap*))

25. MeSH descriptor LYMPHATIC IRRADIATION explode all trees

26. (combi* near/3 modalit*)

27. (\#13 OR \#14 OR \#15 OR \#16 OR \#17 OR \#18 OR \#19 OR \#20 OR \#21 OR \#22 OR \#23 OR \#24 OR \#25 OR \#26)

28. (\#5 AND \#11 AND \#27)

The search strategy was modified the following updated search strategy was also used and will be used for further updates:

\begin{tabular}{|c|c|}
\hline$\# 1$ & (favourable or favorable or unfavourable or unfavorable) \\
\hline \#2 & (I-II or I-III) \\
\hline \#3 & $\left(e^{*} l^{*}\right.$ near $\left./ 3 \operatorname{grad}^{*}\right)$ or $\left(e a r l * n e a r / 3 \mathrm{stag}^{*}\right)$ or $\left(\mathrm{low}^{*}\right.$ near $\left./ 3 \mathrm{stag}^{*}\right)$ or $\left(\mathrm{low}^{*}\right.$ near $\left./ 3 \mathrm{grad}^{*}\right)$ \\
\hline \#4 & (limit* near $/ 3$ stag*) or $\left(\right.$ limit* near $\left./ 3 \operatorname{grad}^{*}\right)$ \\
\hline \#5 & (intermediate*) \\
\hline$\# 6$ & (bulky) \\
\hline \#7 & (\#1 OR \#2 OR \#3 OR \#4 OR \#5 OR \#6) \\
\hline$\# 8$ & MeSH descriptor Lymphoma explode all trees \\
\hline$\# 9$ & MeSH descriptor Hodgkin Disease explode all trees \\
\hline$\# 10$ & (Germinoblastom* or Reticulolymphosarcom*) \\
\hline$\# 11$ & (hodgkin*) \\
\hline$\# 12$ & (malignan* near/2 lymphogranulom*) or (malignan* near/2 granulom*) \\
\hline$\# 13$ & (\#8 OR \#9 OR \#10 OR \#11 OR \#12) \\
\hline$\# 14$ & MeSH descriptor Antineoplastic Agents explode all trees \\
\hline \#15 & MeSH descriptor Remission Induction explode all trees \\
\hline \#16 & MeSH descriptor Antineoplastic Protocols explode all trees \\
\hline \#17 & (consolidat* and therap $\left.{ }^{*}\right)$ or (consolidat* and treat $\left.{ }^{*}\right)$ or (consolidat ${ }^{*}$ and regimen $\left.{ }^{*}\right)$ or (consolidat ${ }^{*}$ and patient $\left.{ }^{*}\right)$ \\
\hline \#18 & (induct* and therap*) or (induct* and treat*) or (induct* and regimen*) or (induct* and patient*) \\
\hline \#19 & (maintenance* and therap*) or (maintenance* and treat* ${ }^{*}$ ) or (maintenance* and regimen*) or (maintenance* and patient $\left.{ }^{*}\right)$ \\
\hline
\end{tabular}


\#21 (therap* near/3 remission*) or (induc* near/3 remission*)

\#22 (chemotherap* or chemo-therap*)

\#23 (Antineoplast* or Anti-neoplast*)

\#24 (cytosta* near/2 therap* ${ }^{*}$ or (cytosta* near/2 treat $\left.{ }^{*}\right)$ or (cytosta* near/2 regimen*)

\#25 (cytotox* near/2 therap*) or (cytotox* near/2 treat*) or (cytotox* near/2 regimen*)

\#26 (\#14 OR \#15 OR \#16 OR \#17 OR \#18 OR \#19 OR \#20 OR \#21 OR \#22 OR \#23 OR \#24 OR \#25)

\#27 MeSH descriptor Radiotherapy explode all trees

\#28 (radiotherap* ${ }^{*}$ or radio-therap*)

\#29 MeSH descriptor Lymphatic Irradiation explode all trees

\#30 (\#27 OR \#28 OR \#29)

\#31 (\#26 AND \#30)

\#32 (chemoradiotherap*) or (chemo-radiotherap*)

\#33 MeSH descriptor Combined Modality Therapy explode all trees

\#34 (multimodal* ${ }^{*}$ ear/3 therap*) or (multimodal* near/3 treat*) or (multi-modal ${ }^{*}$ near/3 therap*) or (multi-modal* ${ }^{*}$ near $/ 3$ treat ${ }^{*}$ )

\#35 (combi* near/3 modalit*)

\#36 (\#32 OR \#33 OR \#34 OR \#35)

\#37 (\#31 OR \#36)

\#38 (\#13 AND \#37)

\#39 (\#7 AND \#13 AND \#37)

\#40 (\#39), from 2008 to 2009 


\section{Appendix 2. MEDLINE search strategy}

The following search strategy was used to search MEDLINE:

1. (favourable or unfavourable).tw,kf,ot.

2. ((earl\$ or low\$ or limit\$) adj3 (stag\$ or grad\$)).tw,kf,ot.

3. intermediate $\$ . t w, k f, o t$.

4. bulky.tw,kf,ot.

5. or/ $1-4$

6. *LYMPHOMA/

7. exp HODGKIN DISEASE/

8. Germinoblastom\$.tw,kf,ot.

9. Reticulolymphosarcom\$.tw,kf,ot.

10. Hodgkin\$.tw,kf,ot.

11. (malignan\$ adj2 (lymphogranulom\$ or granulom\$)).tw,kf,ot.

12. or/6-11

13. $\exp$ ANTINEOPLASTIC AGENTS/

14. REMISSION INDUCTION/

15. exp ANTINEOPLASTIC PROTOCOLS/

16. ((consolidat\$ or induct\$ or maintenance or conditioning $\$)$ and (therap\$ or treat $\$$ or regimen $\$$ or patient $\$)$ ).tw,kf,ot.

17. ((therap\$ or induc\$) adj3 remission\$).tw,kf,ot.

18. (chemotherap\$ or chemo-therap\$).tw,kf,ot.

19. (Antineoplast $\$$ or anti-neoplast $\$)$.tw,kf,ot.

20. ((cytosta $\$$ or cytotox $\$)$ adj2 (therap\$ or treat $\$$ or regimen $\$))$.tw, kf,ot.

21. exp RADIOTHERAPY/

22. (radiotherap\$ or radio-therap\$).tw,kf,ot.

23. (chemoradiotherap\$ or chemo-radio-therap\$).tw,kf,ot.

24. exp COMBINED MODALITY THERAPY/

25. ((multimodal\$ or multi-modal\$) adj3 (treat\$ or therap\$)).tw,kf,ot.

26. exp LYMPHATIC IRRADIATION/

27. (combi\$ adj3 modalit\$).tw,kf,ot.

28. or/13-27

29. randomized controlled trial.pt.

30. controlled clinical trial.pt.

31. RANDOMIZED CONTROLLED TRIALS/

32. RANDOM ALLOCATION/

33. DOUBLE BLIND METHOD/

34. SINGLE BLIND METHOD/

35. or/29-34

36. (ANIMALS not HUMANS).sh.

37. 35 not 36

38. clinical trial.pt.

39. exp CLINICAL TRIALS/

40. (clin\$ adj25 trial\$).ti,ab.

41. ((singl\$ or doubl\$ or trebl\$ or tripl\$) adj25 (blind\$ or mask\$)).ti,ab.

42. PLACEBOS/

43. placebo\$.ti,ab.

44. random \$.ti,ab.

45. RESEARCH DESIGN/

46. or/38-45

47. 46 not 36

48. 47 not 37

49. COMPARATIVE STUDY/

50. $\exp$ EVALUATION STUDIES/

Chemotherapy alone versus chemotherapy plus radiotherapy for early stage Hodgkin lymphoma (Review) 
51. FOLLOW UP STUDIES/

52. PROSPECTIVE STUDIES/

53. (control\$ or prospectiv\$ or volunteer\$).ti,ab.

54. or $/ 49-53$

$55.54 \operatorname{not} 36$

56.55 not $(37$ or 48$)$

57.37 or 48 or 56

58. 5 and 12 and 28 and 57

The search strategy was updated during the review process and the following search strategy was used in addition the the above one. For updates the following search strategy will be used:

1 (favo?rable or unfavo?rable).tw,kf,ot.

2 (I-II or I-III).tw,kf,ot.

3 ((earl\$ or low\$ or limit\$) adj3 (stag\$ or grad\$)).tw,kf,ot.

$4 \quad$ intermediate $\$ . t w, k f, o t$.

5 bulky.tw,kf,ot.

6 or $/ 1-4$

$7 \quad$ *Lymphoma/

8 exp Hodgkin Disease/

$9 \quad$ Germinoblastom\$.tw,kf,ot.

10 Reticulolymphosarcom\$.tw,kf,ot.

11 Hodgkin\$.tw,kf,ot.

12 (malignan\$ adj2 (lymphogranulom\$ or granulom\$)).tw,kf,ot.

13 or $/ 7-12$

14 exp Antineoplastic Agents/

15 Remission Induction/

16 exp antineoplastic protocols/

17 ((consolidat\$ or induct\$ or maintenance or conditioning\$) and (therap\$ or treat $\$$ or regimen $\$$ or patient $\$)$ ).tw, kf,ot.

18 ((therap\$ or induc\$) adj3 remission\$).tw,kf,ot.

19 (chemotherap\$ or chemo-therap\$).tw,kf,ot. 
20 (Antineoplast\$ or anti-neoplast\$).tw,kf,ot.

21 ((cytosta $\$$ or cytotox\$) adj2 (therap\$ or treat\$ or regimen\$)).tw,kf,ot.

22 or/14-21

23 exp Radiotherapy/

24 (radiotherap\$ or radio-therap\$).tw,kf,ot.

25 exp Lymphatic Irradiation/

26 or $/ 23-25$

$27 \quad 22$ and 26

28 (chemoradiotherap\$ or chemo-radio-therap\$).tw,kf,ot.

29 exp Combined Modality Therapy/

30 ((multimodal $\$$ or multi-modal\$) adj3 (treat\$ or therap\$)).tw,kf,ot.

31 (combi\$ adj3 modalit\$).tw,kf,ot.

32 or $/ 28-31$

$33 \quad 27$ or 32

$34 \quad 13$ and 33

$35 \quad 13$ and 33 and 6

36 randomized controlled trial.pt.

37 controlled clinical trial.pt.

38 randomized controlled trials/

39 random allocation/

40 double blind method/

41 single blind method/

42 or/36-41

43 (ANIMALS not HUMANS).sh. 


\section{$44 \quad 42$ not 43}

45 clinical trial.pt.

46 exp clinical trial/

47 (clin\$ adj25 trial\$).ti,ab.

48 ((singl\$ or doubl\$ or trebl\$ or tripl\$) adj25 (blind\$ or mask\$)).ti,ab.

49 placebos/

50 placebo\$.ti,ab.

51 random\$.ti,ab.

52 research design/

53 or $/ 45-52$

$54 \quad 53$ not 43

$55 \quad 54$ not 44

56 comparative study/

57 exp evaluation studies/

58 follow up studies/

59 prospective studies/

60 (control\$ or prospectiv\$ or volunteer\$).ti,ab.

61 or $/ 56-60$

$62 \quad 61$ not 43

$63 \quad 62 \operatorname{not}(44$ or 55$)$

6444 or 55 or 63

$65 \quad 13$ and 32 and 64

$66 \quad 65$ and 6

67 limit 66 to ed=20080401-20090204 
68 randomized controlled trial.pt.

69 controlled clinical trial.pt.

70 randomized controlled trials as topic/

71 random allocation/

72 double blind method/

73 single blind method/

74 or $/ 68-73$

75 (ANIMALS not HUMANS).sh.

$76 \quad 74$ not 75

77 clinical trial.pt.

78 exp clinical trial as topic/

79 (clin\$ adj25 trial\$).ti,ab.

80 ((singl\$ or doubl\$ or trebl\$ or tripl\$) adj25 (blind\$ or mask\$)).ti,ab.

81 placebos/

82 placebo\$.ti,ab.

83 random $\$ . t i, a b$.

84 research design/

85 or $/ 77-84$

$86 \quad 85$ not 75

$87 \quad 86 \operatorname{not} 76$

88 comparative study/

89 exp evaluation studies/

90 follow up studies/

91 prospective studies/ 
93 or $/ 88-92$

$94 \quad 93$ not 75

$95 \quad 94 \operatorname{not}(76$ or 87$)$

9676 or 87 or 95

$97 \quad 35$ and 96

98 from 67 keep 1-19

101 limit 66 to ed $=20080101-20090204$

102 from 101 keep 1-28

103 limit 97 to ed $=20080101-20090204$

104 from 103 keep 1-32

\section{Appendix 3. EMBASE search strategy}

The following strategy was used for EMBASE:

1. (favourable or unfavourable or favorable or unfavorable).tw,kf,ot.

2. ((earl\$ or low\$ or limit\$) adj3 (stag\$ or grad\$)).tw,kf,ot.

3. intermediate\$.tw,kf,ot.

4. bulky.tw,kf,ot.

5. or/1-4

6. *LYMPHOMA/

7. exp HODGKIN DISEASE/

8. Germinoblastom\$.tw,kf,ot.

9. Reticulolymphosarcom\$.tw,kf,ot.

10. Hodgkin\$.tw,kf,ot.

11. (malignan\$ adj2 (lymphogranulom\$ or granulom\$)).tw,kf,ot.

12. or/6-11

13. exp ANTINEOPLASTIC AGENT/

14. REMISSION/

15. exp CLINICAL PROTOCOL/

16. ((consolidat\$ or induct $\$$ or maintenance or conditioning $\$)$ and (therap\$ or treat $\$$ or regimen $\$$ or patient $\$)$ ).tw, $\mathrm{kf}$,ot.

17. ((therap\$ or induc\$) adj3 remission\$).tw,kf,ot.

18. (chemotherap\$ or chemo-therap\$).tw,kf,ot.

19. (Antineoplast\$ or anti-neoplast\$).tw,kf,ot.

20. ((cytosta $\$$ or cytotox\$) adj2 (therap\$ or treat\$ or regimen\$)).tw,kf,ot.

21. exp RADIOTHERAPYI

22. (radiotherap\$ or radio-therap\$).tw,kf,ot.

23. (chemoradiotherap\$ or chemo-radio-therap\$).tw,kf,ot. 
24. exp MULTIMODALITY CANCER THERAPY/

25. ((multimodal\$ or multi-modal\$) adj3 (treat\$ or therap\$)).tw,kf,ot.

26. exp LYMPH NODE IRRADIATION/

27. (combi\$ adj3 modalit\$).tw,kf,ot.

28. or/13-27

29. CLINICAL TRIAL/

30. RANDOMIZED CONTROLLED TRIALS/

31. RANDOM ALLOCATION/

32. SINGLE-BLIND METHOD/

33. DOUBLE-BLIND METHOD/

34. CROSS-OVER STUDIES/

35. PLACEBOS/

36. Randomi?ed controlled trial\$.tw.

37. RCT.tw.

38. Random allocation.tw.

39. Randomly allocated.tw.

40. Allocated randomly.tw.

41. (allocated adj2 random).tw.

42. Single blind\$.tw.

43. Double blind\$.tw.

44. ((treble or triple) adj blind $\$)$.tw.

45. Placebo\$.tw.

46. PROSPECTIVE STUDIES/

47. or/29-46

48 CASE STUDY/

49. Case report.tw.

50. ABSTRACT REPORT/ or LETTER/

51. or $/ 48-50$

52. 47 not 51

53. ANIMAL/

54. HUMAN/

55.53 not 54

56.52 not 55

57.5 and 12 and 28 and 56

\section{WHAT'S NEW}

Last assessed as up-to-date: 1 January 2011.

\begin{tabular}{l|l|l}
\hline Date & Event & Description \\
\hline 8 February 2011 & Amended & Typo correction \\
\hline
\end{tabular}




\section{H I S T O R Y}

Protocol first published: Issue 2, 2008

Review first published: Issue 2, 2011

\section{CONTRIBUTIONS OFAUTHORS}

Herbst C: Abstract screening, data extraction, quality assessment (RoB), data analysis and interpretation, drafting of the review, SoF table, adverse events

Rehan F: Drafting of the protocol, abstract screening, data extraction, data entry into RevMan, drafting of the review

Skoetz N: Data checking (third author), communication between authors, proofreading, update screening

Brillant C: Data extraction, data analysis and interpretation, statistical advice

Bohlius J: Conception of the review, drafting of the protocol, methodological advice

Schulz H: Clinical expertise

Monsef I: Search strategy, electronic search, handsearching

Specht L: Clinical expertise, advice for the protocol

Engert A: Clinical expertise, content input

\section{DECLARATIONS OF INTEREST}

There is no known conflict of interest.

\section{SOURCES OF SUPPORT}

\section{Internal sources}

- Köln Fortune, Germany.

Funding programme "Köln Fortune", Medical Faculty University of Cologne

\section{External sources}

- BMBF, Germany.

Project grant application NO 01KG0815, Federal Ministry of Education and Research (BMBF)

\section{DIFFERENCES BETWEEN PROTOCOLAND REVIEW}




\section{Assessment of risk of bias in included studies}

For quality assessment we preferred to use a "domain-based evaluation” as described in The Cochrane Collaboration's tool for assessing risk of bias (Higgins 2008), since it was more compatible to the risk of bias table included in the RevMan 5. We replaced the following quality questions:

- Was treatment allocation concealed?

- Were outcome assessors blind to treatment assigned?

- Were numbers of withdraws, drop outs, lost to follow-up and protocol violations in each group stated and were less than $10 \%$ in each arm?

- Were participants included in the analyses as part of the group to which they were allocated (intention-to-treat analyses)?

- Were the baseline characteristics similar in both groups?

\section{Progression-free survival}

Because no trials reported progression-free survival (PFS) according to our definition (time to progress or relapse or death of any cause in all randomised patients), we accepted other progression outcomes and evaluated these as tumour control.

\section{Adverse events}

It was not planned to extract adverse events because the adverse events relevant for decision making were not expected to be reported in the reviews. To further underline this point, all adverse events reported in the review were summarised in a table.

\section{Summary of Findings Table}

A Summary of Findings Table using the GRADE approach was included.

\section{N DEX TERMS}

\section{Medical Subject Headings (MeSH)}

Combined Modality Therapy [methods]; Hodgkin Disease [*drug therapy; pathology; ${ }^{*}$ radiotherapy]; Randomized Controlled Trials as Topic; Survival Analysis

\section{MeSH check words}

Humans 